\title{
Description and Analysis of the Second Spectrum of Molybdenum, Mo II
}

\author{
C. C. Kiess
}

\begin{abstract}
Wavelengths and estimated intensities are presented for 3,800 lines of Mo II in the spectral range from $6100 \mathrm{~A}$ in the red to $1550 \mathrm{~A}$ in the ultraviolet. For approximately 970 of these lines, Zeeman patterns have been measured. Analysis of these spectral data shows that about 70 percent of the lines can be accounted for as transitions between levels of the even terms in the electron configurations $4 d^{5}$ and $4 d^{4} 5 s$, and levels of the odd terms in the configuration $4 d^{4} 5 \mathrm{p}$. No series have been found from which an ionization potential can be derived.
\end{abstract}

\section{Introduction}

The description and analysis of the second spectrum of molybdenum presented in this paper are the results of investigations that for more than three decades have been in progress at the National Bureau of Standards. Work on the analysis of the molybdenum spectra was begun here shortly after similar work on the chromium spectra had been initiated, when it was realized that spectral structures like those of chromium should occur also in the spectra of its homologue molybdenum. A brief search through the spectroscopic data available at that time for molybdenum readily brought to light the expected structures. Subsequently these were published [1] ${ }^{1}$ in illustration of the operation of the spectroscopic displacement and alternation laws among the elements of the second long period of Mendeléeff's table.

This earlier phase of the work demonstrated the need for better descriptions of the molybdenum spectra than existed at that time. Many of the weaker lines, which are essential to the thorough analysis of a rich-lined spectrum, were missing from the published wavelength lists; and the intensities of the lines, estimated on a compressed scale, were inadequate for the finer discrimination of apparently related spectral regularities. Such Zeeman-effect observations as were available extended over only a limited range of wavelengths and, with a few exceptions, gave unresolved patterns for many of the lines. In view of these circumstances, it was decided to make a new description of the molybdenum spectra throughout the range accessible to photographic recording with the spectrographs at the Bureau.

\section{Experimental Procedure}

The spectrographic equipment of the Bureau has been described in detail in earlier papers [2]. The three concave gratings, of $21-\mathrm{ft}$ radius of curvature, used in this work, were the ones ruled with 7,500 , 20,000, and 30,000 lines/in. by J. A. Anderson, H. A. Rowland, and R. W. Wood, respectively. They were mounted according to the Wadsworth style, and recorded the spectra from $1975 \mathrm{~A}$ in the ultraviolet to $12000 \mathrm{~A}$ in the infrared. First-order spectra

\footnotetext{
1 Figures in brackets indicate the literature references at the end of this paper.
}

were obtained with each of the gratings, but, whereever possible, second- and, in a few cases, third-order spectra also were obtained with the Rowland and Wood gratings. The first-order dispersions of these gratings are $10,3.5$, and $2 \mathrm{~A} / \mathrm{mm}$, respectively. A set of spectrograms covering the region shortward of 3000 A was obtained with the quartz-prism spectrographs. Hilger's E 1 instrument was used for the range 2150 to $1975 \mathrm{~A}$, with an average dispersion of $1 \mathrm{~A} / \mathrm{mm}$; the E 185 instrument was used for wavelengths from 3000 to $2100 \mathrm{~A}$, with dispersion ranging from $1 \mathrm{~A} / \mathrm{mm}$ to $0.3 \mathrm{~A} / \mathrm{mm}$.

The plates for recording the spectra were selected from the varieties offered in the Eastman Kodak Co. (EK) catalog. For the earlier observations, the EK 33 emulsions were used, sensitized in dye solutions, when necessary, for the longer wavelength regions beyond the reach of ordinary plates. For the later observations, the EK sensitizings appropriate to the various spectral regions were used. For the ultraviolet shorter than 2300 A, Schumann plates supplied by Hilger were used.

The electrodes for both the arc and spark exposures were cut from rods of very pure molybdenum metal about $5 \mathrm{~mm}$ in diameter. To the Research Laboratory of the General Electric Co., we are indebted for the electrode material used in the earlier observations; and to the Climax Molybdenum Co. for the material used in the later series of observations. The only impurities detected were traces of iron, and possibly silicon.

For the arc spectra, the exposures were made to arcs-in-air, and to ares in an enclosed chamber, of the type described by Curtis [3], in which the pressure could be reduced or in which an atmosphere of inert gases could be maintained. The ares were operated at 5 or 6 amp from $220-\mathrm{v}$ d-c mains. For the spark spectra, the exposures were made to sparksin-air, or in the enclosed chamber used in the work on chromium. The spark discharges were supplied by a battery of condensers of $0.006 \mu \mathrm{f}$ capacitance that was charged up to $30,000 \mathrm{v}$ from the secondary coil of a transformer of which the primary coil carried $110-\mathrm{v}$ ac. In the case of the arc spectrum, the object of enclosing the arc was to reduce the intensity of the molybdenum oxide bands that black out a considerable portion of the spectrum when the arc is operated in air. In the case of the spark 
spectrum, enclosure of the spark in an inert gas or in air at lowered pressure reduced the intensity and diffuseness of the atmospheric lines that are always present in spectrograms of a spark in air. In both cases, enclosure of the light source at reduced pressure sharpens the spectral lines, which enhances the accuracy of measurement of lines that are diffuse or asymmetrical. All exposures to the molybdenum sources were made in juxtaposition to exposures of the iron arc as the source of the standards for wavelength determinations. In the extreme ultraviolet, where the lines of iron are weak or insufficient in number, electrodes of coppersilver alloy were used for the comparison spectrum. The wavelengths of the standard copper lines were those published by Burns and Walters [4].

Measurements of the plates, on which this investigation is based, were made by the author in cooperation with C. L. Zimmerman [5]. In addition to the spectrograms made at the Bureau, there were available for measurement several spectrograms of the region shortward of $2000 \mathrm{~A}$ made with vacuum spectrographs, by A. G. Shenstone at Princeton, University, and J. C. Boyce at the Massachusetts Institute of Technology (MIT). The scale of the plates obtained with these spectrographs is practically the same, about $4.2 \mathrm{~A} / \mathrm{mm}$.

In addition to an accurate and homogeneous set of wavelengths and reliable estimates of intensities, an analysis of a complex spectrum requires also a knowledge of the patterns of the lines in magnetic fields. Two sets of observations of the Zeeman effect of molybdenum have been made for this investigation. One set of plates covering the spectral range from $2250 \mathrm{~A}$ to $7500 \mathrm{~A}$ was made with the water-cooled Weiss magnet of the Bureau. In a pole gap of $6 \mathrm{~mm}$, a field of 35,000 oersteds was attained with a current of $180 \mathrm{amp}$ in the coils under a potential of $90 \mathrm{v}$. The other set of Zeemaneffect plates was obtained with the Bitter magnet and the spectrographs of MIT. This equipment has been adequately described by G. R. Harrison $[6,7]$ who kindly sent us the molybdenum plates for this investigation. These plates, taken with fields of 86,000 oersteds, cover the range from 2180 A to $8180 \mathrm{~A}$. All the plates of both sets were measured by the author.

\section{Results}

\subsection{Wavelengths and Intensities}

The new wavelengths and estimated intensities for lines characteristic of Mo II are recorded in the first two columns of tables 1 and 2 . The values adopted for the wavelengths of table 1 are the means of two or more measurements and, in general, are given to the nearest $0.01 \mathrm{~A}$. In many cases, however, for which more than two highly accordant measurements were made, the third decimal place of the mean value has been retained. All the wavelengths in table 1 are their values in air. All the lines listed in table 2 were measured on the spectrograms made with the vacuum spectrographs; but the wavelengths assigned to them are the values calculated from combinations between the terms derived from the analysis of the lines in table 1 .

The numbers and letters in the second column of the tables indicate the relative strengths and characteristics of the lines. There are no photometricallydetermined intensities known for lines of Mo II, so that it is not possible to compare the visual estimates with measured values. However, there are available at the Bureau, some unpublished estimated intensities made by Meggers for lines emitted by molybdenum arcs relative to lines of calibrated intensity in the copper arc. The lines of Mo II included in his list show a good correlation with the estimated intensities reported in this work, if due allowance is made for differences in mode of excitation.

The letters following the intensities have the following meanings: $d=$ double; $e=$ enhanced at the electrode; $h=$ hazy $H=$ very hazy $l=$ shaded longward ; $s=$ shaded shortward $w=$ wide; $W=$ very wide. Lines designated $w$ are probably unresolved pairs, and lack the appearance of diffuseness that is characteristic of those marked $h$. The letter $\mathrm{Z}$ means that a Zeeman pattern, measured on NBS or MIT spectrograms, is recorded for the line in table 3 .

\subsection{Zeeman Effects}

The Zeeman patterns for about 970 lines of Mo II are given in table 3 . A dagger ( $\dagger$ ) indicates that the pattern was measured only on NBS spectrograms; the letters $A, B, C$, and $D$ indicate the type of shading displayed by unresolved patterns, thus: $A=\angle \|$; $B=\backslash ; C=\wedge ; D=\sqcap$. The only Zeeman patterns published heretofore for lines of molybdenum are those by Jack [8] and by Wilhelmy [9]. Of the 150 lines measured by Jack, in a field of approximately 25,000 oersteds, about one-third belong to Mo II. His fully resolved patterns are in good agreement with corresponding ones in table 3 . Wilhelmy, using a higher field of approximately 35,000 oersteds, likewise got magnetic patterns for about 55 lines of Mo II and thereby established the identity of the low ${ }^{6} \mathrm{D}$ term of the ion. His results also are in very good agreement with those of table 3 .

\subsection{Term Structures of Mo II}

The first multiplets of Mo II were announced in 1926, by Meggers and Kiess [1], as part of the evidence for the operation of the spectroscopic displacement law in the second long period of the periodic table. Although the unraveling of the spectrum bas continued steadily since that time, no other results have been reported from the Bureau. In the meantime, additional terms and classified lines have been announced by Schauls and Sawyer [10] and by V. R. Rao [11]. Some erroneous designations of levels occur, in each of these earlier investigations, which, it is believed, the present analysis corrects.

Theoretically, the singly ionized atom of molybdenum is isoelectronic with the neutral atom of niobium and, therefore, in accordance with the displacement law, the spectra of the two atoms should be similar. Although the term structures of these spectra arise in electron configurations of the same type yet, 
owing to differences in the relative i mportance o these configurations, the spectra $\mathrm{NbI}$ and Mo II have the appearance of being strikingly dissimilar. In atoms with 41 external electrons, 36 are in closed shells. The 5 outermost electrons, which determine the optical properties of the atoms, may assume, on excitation, the various configurational groupings that govern the distribution of radiant energy in the spectrum. The low, even states of the atom are given by the configurations $4 d^{5}, 4 d^{4} 5 s$, and $4 d^{3} 5 s^{2}$, whereas the higher, excited, odd states arise in the configurations $4 d^{4} 5 p$ and $4 d^{3} 5 s 5 p$. In $\mathrm{Nb}$ I the group $4 d^{5}$ is the least important of the low, even configurations, but in Mo II it is first in importance and $4 d^{3} 5 s^{2}$ is least. Of the odd configurations, only $4 d^{4} 5 p$ contributes to Mo II, whereas both share prominently in $\mathrm{Nb} \mathrm{I}$.

The even and odd terms of Mo II, and the electron configurations in which they arise, are recorded in tables 4 and 5. All but the two highest terms of the configuration $4 d^{5}$ have been found. In the configuration $4 d^{4} 5 s$, all the quartets except the high ${ }^{4} \mathrm{P}$, and most of the doublets have been found. None of the terms of the configuration $4 d^{3} 5 s^{2}$, whicb is so prominent in $\mathrm{Nb}$, have been located. The only odd terms that have been established belong to the configuration $4 d^{4} 5 p$. Table 6 lists the terms that are theoretically possible for Mo II, those actually found being designated in bold-faced type. The $g$ values assigned to each level are, in general, the means of several determinations.

The assignment of the various terms to the electron configurations to which they belong presents no difficulty for the lower members of a configurational group. In general, the intervals between the levels of the $4 d^{5}$ terms are smaller than those of the $4 d^{4} 5 s$ terms, and exhibit partial inversions. But in the higher members of a family of terms, these characteristics are not so evident, and reliance has been placed on theoretical calculations of the origin and energy of the expected terms. Such calculations have been made for this investigation by Goldgraber [12] for the low, even terms of the configurations $4 d^{5}$ and $4 d^{4} 5 s$, and by Trees [13] for the odd $4 d^{4} 5 p$ terms.

\subsection{Series and Ionization Potential}

No series of two or more members have been found in Mo II, and therefore it is not yet possible to calculate an ionization potential for the singly ionized molybdenum atom from experimental data derived from the atom itself. But from similarities that exist in the term structure of neighboring atoms for which good spectral series have been worked out, it is possible to derive closely approximate values for series limits and ionization potentials for atoms for which these values are not directly available. This has been done by Catalán and Rico [14] for some of the neutral and singly ionized atoms of the second long period of elements. In a subsequent revision of this work they [15] give $130,300 \mathrm{~cm}^{-1}$ for the separation of the ground states of Mo II and Mo III, which corresponds to an ionization potential of $16.15 \mathrm{ev}$.

Over the long period during which this work has been in progress, it has been aided in various ways by several individuals. A. G. Shenstone and J. C. Boyce have sent their spectrograms of the extreme ultraviolet spectra of molybdenum, and G. R. Harrison has sent his spectrograms of the Zeeman patterns. C. L. Zimmerman measured and reduced most of the spectrograms of the ultraviolet region. In the reductions of many of the other spectrograms, Miss M. M. Harvey and W. Lyle took part. Finally, H. D. Goldgraber and R. E. Trees contributed greatly to the theoretical interpretation and configurational assignment of the energy states. To each of them it is a pleasure to express appreciation for contributions to this work.

\section{References}

[1] W. F. Meggers and C. C. Kiess, J. Opt. Soc. Am. 12, 417 (1926).

[2] W. F. Meggers and K. Burns, BS Sci. Pap. 18, 191 (1922) S441.

[3] H. D. Curtis, J. Opt. Soc. Am. 8, 697 (1924).

[4] K. Burns and F. M. Walters, Jr., Publ. Allegheny Observatory, Univ. Pittsburgh 8, 27 (1930)

[5] C. L. Zimmerman, Dissertation (Duke Univ., Durham, N. C., 1937).

[6] G. R. Harrison and F. Bitter, Phys. Rev. 57, 15 (1940).

[7] G. R. Harrison and J. R. MeNally, Jr., Phys. Rev. 58, $703(1940)$.

[8] R. Jack, Ann. Physik IV 28, 1032 (1909)

[9] E. Wilhelmy, Ann. Physik IV 80, 305 (1926).

[10] R. Schauls and R. A. Sawyer, Phys. Rev. 58, 781 (1940).

[11] V. R. Rao, Indian J. Phys. 23, 387 (1949).

[12] H. D. Goldgraber, University of Pennsylvania dissertation (1953).

[13] R. E. Trees (private communication).

[14] M. A. Catalán and F. R. Rico, An. Españ. Soc. Fis. Quim. [A] 48, 328 (1952).

[15] F. R. Rico (letter, dated Dec. 12, 1956).

TABLE 1. Wavelengths and term combinations of Mo II

\begin{tabular}{|c|c|c|c|c|c|c|c|}
\hline $\begin{array}{c}\text { Wavelength } \\
\lambda_{\text {air }}\end{array}$ & Intensity & Wave No. & $\begin{array}{c}\text { Term } \\
\text { combination }\end{array}$ & $\begin{array}{c}\text { Wavelength } \\
\lambda_{\text {air }}\end{array}$ & Intensity & Wave No. & $\begin{array}{c}\text { Term } \\
\text { combination }\end{array}$ \\
\hline $\begin{array}{l}6083.68 \\
5914.066 \\
5905.348 \\
5885.121 \\
5871.014 \\
\\
5862.68 \\
5862.22 \\
5851.76\end{array}$ & $\begin{array}{r}1 \\
8 \\
20 \\
10 \\
1 \\
1 \\
1 \\
3\end{array}$ & $\begin{array}{l}16432.88 \\
16904.17 \\
16929.12 \\
16987.31 \\
17028.12 \\
17052.33 \\
17053.67 \\
17084.15\end{array}$ & $\begin{array}{l}c^{4} \mathrm{D}_{21 / 2}-z^{6} \mathrm{D}_{31 / 2}^{\circ} \\
c^{4} \mathrm{~F}_{41 / 2}-z^{4} \mathrm{G}_{51 / 2}^{\circ} \\
c^{4} \mathrm{D}_{31 / 2}-z^{6} \mathrm{D}_{21 / 2}^{\circ} \\
c^{4} \mathrm{D}_{11 / 2}-z^{6} \mathrm{D}_{21 / 2}^{\circ}\end{array}$ & $\begin{array}{l}5827.136 \\
5788.455 \\
5775.674 \\
5774.314 \\
5754.46\end{array}$ & $\begin{array}{l}7 \\
3 \\
3 \\
2 \\
2\end{array}$ & $\begin{array}{l}\text { 17131. } 67 \\
17143.22 \\
\\
17156.33 \\
17270.98 \\
17309.20 \\
17313.27 \\
17373.01\end{array}$ & $\begin{array}{c}d^{2} \mathrm{~F}_{31 / 2}-z^{2} \mathrm{G}_{31 / 2}^{\circ} \\
\left\{\begin{array}{l}b^{2} \mathrm{G}_{31 / 2}-z^{6} \mathrm{D}_{31 / 2}^{\circ} \\
c^{4} \mathrm{D}_{31 / 2}-z^{6} \mathrm{D}_{41 / 2}^{\circ}\end{array}\right. \\
b^{4} \mathrm{~F}_{21 / 2}-z^{6} \mathrm{~F}_{11 / 2}^{\circ} \\
d^{2} \mathrm{G}_{31 / 2}-z^{4} \mathrm{G}_{31 / 2}^{1} \\
a^{2} \mathrm{P}_{11 / 2}-z^{4} \mathrm{~F}_{21 / 2}^{\circ} \\
d^{2} \mathrm{G}_{41 / 2}-z^{4} \mathrm{I}_{41 / 2}^{\circ}\end{array}$ \\
\hline
\end{tabular}


TABLE 1. Wavelengths and term combinations of Mo II-Continued

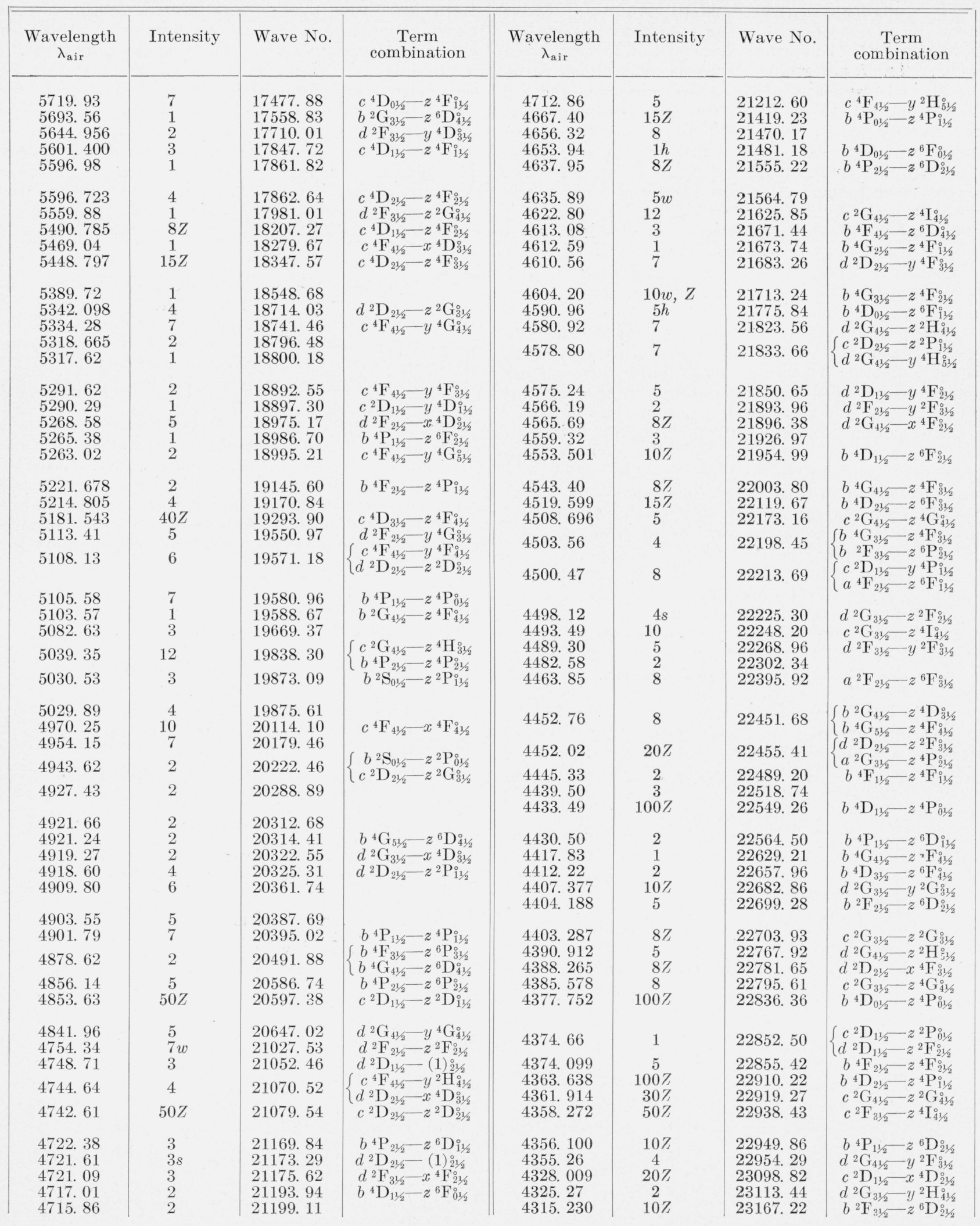


TABLE 1. Wavelengths and term combinations of Mo II-Continued

\begin{tabular}{|c|c|c|c|c|c|c|c|}
\hline $\begin{array}{c}\text { Wavelength } \\
\lambda_{\mathrm{air}}\end{array}$ & Intensity & Wave No. & $\begin{array}{c}\text { Term } \\
\text { combination }\end{array}$ & $\begin{array}{c}\text { Wavelength } \\
\lambda_{\text {air }}\end{array}$ & Intensity & Wave No. & $\begin{array}{c}\text { Term } \\
\text { combination }\end{array}$ \\
\hline $\begin{array}{l}\text { 4312. } 46 \\
4312.38 \\
4311.649 \\
4311.041 \\
4310.63\end{array}$ & $\begin{array}{l}3 \\
1 \\
407 \\
407 \\
2\end{array}$ & $\begin{array}{l}23182.10 \\
23182.53 \\
23186.46 \\
23189.73 \\
23191.94\end{array}$ & $\begin{array}{l}c^{2} \mathrm{D}_{11 / 2}-y^{4} \mathrm{P}_{21 / 2}^{\circ} \\
b^{4} \mathrm{~F}_{41 / 2}-z^{4} \mathrm{~F}_{31 / 2}^{0} \\
a^{2} \mathrm{~F}_{21 / 2}-z^{4} \mathrm{P}_{11 / 2}^{\circ} \\
c^{2} \mathrm{~F}_{21 / 2}-z^{4} \mathrm{G}_{31 / 2}^{0} \\
c^{2} \mathrm{D}_{21 / 2}-y^{4} \mathrm{~F}_{31 / 2}^{\circ}\end{array}$ & $\begin{array}{l}4108.657 \\
4106.60 \\
4105.44 \\
4100.303 \\
4098.46\end{array}$ & $\begin{array}{l}2 \\
1 \\
3 \\
57 \\
1\end{array}$ & $\begin{array}{l}24332.01 \\
24344.20 \\
24351.08 \\
24381.58 \\
24392.55\end{array}$ & $\begin{array}{c}a^{2} \mathrm{D}_{11 / 2} z^{4} \mathrm{P}_{01 / 2}^{\circ} \\
a^{2} \mathrm{~F}_{31 / 2}-z^{4} \mathrm{P}_{21 / 2}^{0} \\
b^{4} \mathrm{D}_{11 / 2}-z^{6} \mathrm{P}_{11 / 2}^{0} \\
b^{2} \mathrm{I}_{61 / 2}-z^{4} \mathrm{H}_{11 / 2}^{1}\end{array}$ \\
\hline $\begin{array}{l}4303.87 \\
4291.53 \\
4283.28 \\
4281.22 \\
4280.57\end{array}$ & $\begin{array}{r}4 \\
2 l \\
4 \\
10 \\
2\end{array}$ & $\begin{array}{l}23228.37 \\
23295.16 \\
23340.05 \\
23351.26 \\
23354.80\end{array}$ & $\begin{array}{l}b^{4} \mathrm{~F}_{31 / 2}-z^{4} \mathrm{~F}_{31 / 2}^{\circ} \\
b^{2} \mathrm{~F}_{31 / 2}-z^{6} \mathrm{D}_{41 / 2}^{\circ} \\
b^{4} \mathrm{~F}_{21 / 2}-z^{4} \mathrm{~F}_{31 / 2}^{\circ} \\
c^{2} \mathrm{D}_{11 / 2}(1)^{\circ}{ }^{1112} \\
b^{2} \mathrm{I}_{51 / 2}-z^{4} \mathrm{H}_{51 / 2}^{\circ}\end{array}$ & $\begin{array}{l}\text { 4095. } 55 \\
\text { 4094. } 965 \\
\text { 4090. } 994 \\
\text { 4084. } 00 \\
\text { 4082. } 035\end{array}$ & $\begin{array}{c}2 \\
107 \\
3 \\
2 h \\
2\end{array}$ & $\begin{array}{l}24409.88 \\
24413.37 \\
24437.06 \\
24478.89 \\
24490.67\end{array}$ & $\begin{array}{l}\left\{\begin{array}{l}d^{2} \mathrm{D}_{21 / 2}-(3)_{31 / 2}^{\circ} \\
d^{2} \mathrm{G}_{41 / 2}-w^{4} \mathrm{D}_{31 / 2}^{\circ}\end{array}\right. \\
c^{2} \mathrm{~F}_{21 / 2}-y^{4} \mathrm{D}_{31 / 2}^{3} \\
d^{2} \mathrm{G}_{41 / 2}-x^{4} \mathrm{G}_{51 / 2}^{\circ}\end{array}$ \\
\hline $\begin{array}{l}4279.02 \\
4274.441 \\
4267.83 \\
4266.67 \\
4256.67\end{array}$ & $\begin{array}{c}507 \\
207 \\
1 \\
5 \\
10\end{array}$ & $\begin{array}{l}\text { 23363. } 26 \\
23388.29 \\
23424.52 \\
23430.89 \\
23485.93\end{array}$ & $\begin{array}{c}b^{4} \mathrm{D}_{11 / 2}-z^{4} \mathrm{P}_{11 / 2}^{0} \\
d^{2} \mathrm{G}_{41 / 2}-y^{2} \mathrm{G}_{41 / 2}^{0} \\
c^{4} \mathrm{D}_{01 / 2}-y^{4} \mathrm{D}_{01 / 2}^{0} \\
d^{2} \mathrm{D}_{21 / 2}-y^{2} \mathrm{G}_{31 / 2}^{0} \\
c^{2} \mathrm{~F}_{31 / 2}-z^{4} \mathrm{G}_{41 / 2}^{0}\end{array}$ & $\begin{array}{l}4081.070 \\
4077.68 \\
4074.437 \\
4067.835 \\
4058.61\end{array}$ & $\begin{array}{l}107 \\
107 \\
3 \\
3 \\
27\end{array}$ & $\begin{array}{l}24496.46 \\
24516.85 \\
24536.34 \\
24576.16 \\
24632.02\end{array}$ & $\begin{array}{l}b^{4} \mathrm{D}_{21 / 2}-z^{6} \mathrm{P}_{21 / 2}^{\circ} \\
a^{4} \mathrm{H}_{31 / 2}-z^{6} \mathrm{D}_{21 / 2}^{\circ} \\
c^{4} \mathrm{D}_{01 / 2}-z^{2} \mathrm{~S}_{01 / 2}^{\circ}\end{array}$ \\
\hline $\begin{array}{l}4253.38 \\
4250.687 \\
4248.57 \\
4246.58 \\
4244.725\end{array}$ & $\begin{array}{c}4 \\
307 \\
2 \\
107 \\
507\end{array}$ & $\begin{array}{l}23504.10 \\
23519.01 \\
23530.71 \\
23541.73 \\
23552.02\end{array}$ & $\begin{array}{l}b^{4} \mathrm{D}_{31 / 2}-z^{4} \mathrm{P}_{21 / 2}^{\circ} \\
c^{2} \mathrm{G}_{31 / 2}-z^{2} \mathrm{G}_{41 / 2}^{\circ} \\
c^{2} \mathrm{~F}_{21 / 2}-z^{2} \mathrm{D}_{11 / 2}^{\circ}\end{array}$ & $\begin{array}{l}4057.70 \\
4055.60 \\
4053.28 \\
4053.03\end{array}$ & $\begin{array}{l}3 \\
1 \\
1 h \\
1 h\end{array}$ & $\begin{array}{l}24637.54 \\
24650.30 \\
24664.41 \\
24665.93\end{array}$ & $\begin{array}{c}b^{2} \mathrm{G}_{41 / 2}-z^{4} \mathrm{H}_{31 / 2}^{\circ} \\
\left\{\begin{array}{l}a^{4} \mathrm{H}_{31 / 2}-z^{6} \mathrm{D}_{41 / 2}^{\circ} \\
d^{2} \mathrm{D}_{21 / 2}-x^{4} \mathrm{G}_{31 / 2}^{\circ} \\
d^{2}{ }^{2} \mathrm{~F}_{31 / 2}-x^{2} \mathrm{~F}_{21 / 2}^{0}\end{array}\right.\end{array}$ \\
\hline $\begin{array}{l}4243.099 \\
4238.15 \\
4227.073 \\
4209.648 \\
4198.935\end{array}$ & $\begin{array}{l}10 \\
3 \\
127 \\
607 \\
10\end{array}$ & $\begin{array}{l}23561.05 \\
23588.56 \\
23650.37 \\
23748.27 \\
23808.85 \\
23846.69\end{array}$ & $\begin{array}{l}c^{2} \mathrm{G}_{31 / 2}-z^{2} \mathrm{D}_{21 / 2}^{\circ} \\
b^{4} \mathrm{P}_{01 / 2}-z^{6} \mathrm{D}_{11 / 2}^{\circ} \\
b^{4} \mathrm{D}_{01 / 2}-z z^{4} \mathrm{P}_{11 / 2}^{0} \\
b^{4} \mathrm{D}_{21 / 2}-z^{4} \mathrm{P}_{21 / 2}^{\circ} \\
b^{4} \mathrm{~F}_{41 / 2}-z^{4} \mathrm{~F}_{41 / 2}^{0} \\
c^{2} \mathrm{~F}_{21 / 2}-z^{2} \mathrm{G}_{31 / 2}^{0}\end{array}$ & $\begin{array}{l}\text { 4049. } 75 \\
\text { 4048. } 98 \\
4046.84 \\
4036.32 \\
4036.12 \\
4035.566\end{array}$ & $\begin{array}{l}1 \\
2 h \\
2 \\
1 \\
2 \\
5\end{array}$ & $\begin{array}{l}24685.91 \\
\\
24690.60 \\
24703.66 \\
24768.07 \\
24769.27 \\
24772.67\end{array}$ & $\begin{array}{l}c^{2} \mathrm{~F}_{21 / 2}-z^{2} \mathrm{D}_{21 / 2}^{0} \\
d^{2} \mathrm{~F}_{31 / 2}-x^{2} \mathrm{G}_{31 / 2}^{21} \\
b^{4} \mathrm{P}_{01 / 2}-z^{4} \mathrm{~F}_{11 / 2}^{\circ} \\
a^{2} \mathrm{~F}_{21 / 2}-z^{6} \mathrm{P}_{21 / 2}^{\circ}\end{array}$ \\
\hline 4191. 03 & 57 & 23853. 78 & $\left\{\begin{array}{l}b^{2} \mathrm{~F}_{21 / 2}-z^{4} \mathrm{~F}_{21 / 2}^{0} \\
b^{4} \mathrm{~F}_{31 / 2}-z^{4} \mathrm{~F}_{41 / 2}^{0}\end{array}\right.$ & $\begin{array}{l}\text { 4029. } 966 \\
4027.700\end{array}$ & $\begin{array}{l}10 l 7 \\
2\end{array}$ & $\begin{array}{l}24807.09 \\
24821.05\end{array}$ & $\begin{array}{c}b^{2} \mathrm{~F}_{31 / 2}-z^{4} \mathrm{~F}_{31 / 2}^{\circ} \\
b^{2} \mathrm{I}_{51 / 2}-z^{4} \mathrm{G}_{41 / 5}^{0}\end{array}$ \\
\hline $\begin{array}{l}\text { 4177. } 939 \\
\text { 4172. } 280 \\
\text { 4171. } 790\end{array}$ & $\begin{array}{c}5 \\
4 l \\
107\end{array}$ & $\begin{array}{l}23928.50 \\
23960.96 \\
23963.77\end{array}$ & $\begin{array}{c}b^{4} \mathrm{D}_{21 / 2}-z^{6} \mathrm{P}_{11 / 2}^{41 / 2} \\
c^{2} \mathrm{~F}_{31 / 2}-y^{4} \mathrm{D}_{31 / 2}^{\circ} \\
c^{2} \mathrm{D}_{21 / 2}-z^{2} \mathrm{~F}_{31 / 2}^{0}\end{array}$ & $\begin{array}{l}\text { 402. } 01 \\
\text { 4023. } 535 \\
\text { 4016. } 095\end{array}$ & $\begin{array}{c}1 \\
107 \\
3 h\end{array}$ & $\begin{array}{l}24821.03 \\
24837.64 \\
24846.74 \\
24892.77\end{array}$ & $\begin{array}{l}c^{2} \mathrm{D}_{21 / 2}-z^{4} \mathrm{~S}_{11 / 2}^{11 / 2} \\
b^{2} \mathrm{H}_{41 / 2}-z^{4} \mathrm{H}_{31 / 2}^{0}\end{array}$ \\
\hline 4171. 19 & 3 & 23967. 22 & $c^{4} \mathrm{~F}_{41 / 2}-w^{4} \mathrm{~F}_{31 / 2}^{\circ}$ & 4013. 200 & 127 & 24910.73 & $c^{2} \mathrm{G}_{31 / 2}-x^{4} \mathrm{D}_{21 / 2}^{2}$ \\
\hline 4161. 247 & 77 & 24024. 48 & $\left\{\begin{array}{l}a^{2} \mathrm{~F}_{21 / 2}-z^{4} \mathrm{P}_{21 / 2}^{01} \\
d^{2} \mathrm{~F}_{21 / 2}-x^{2} \mathrm{~F}_{31 / 2}^{0}\end{array}\right.$ & & $\begin{array}{l}37 \\
5\end{array}$ & & \\
\hline $\begin{array}{l}\text { 4161. } 02 \\
\text { 4154. } 272 \\
\text { 4152. } 22\end{array}$ & $\begin{array}{l}3 s \\
3 \\
1\end{array}$ & $\begin{array}{l}24025.80 \\
24064.82 \\
24076.71\end{array}$ & & $\begin{array}{l}\text { 4006. } 968 \\
\text { 4005. } 120\end{array}$ & $\begin{array}{l}2 \\
57\end{array}$ & $\begin{array}{l}24949.47 \\
24960.98\end{array}$ & $\begin{array}{l}b^{4} \mathrm{D}_{11 / 2}-z^{6} \mathrm{P}_{21 / 2}^{01 / 2} \\
b^{4} \mathrm{D}_{31 / 2}-z^{6} \mathrm{D}_{31 / 2}^{\circ}\end{array}$ \\
\hline $\begin{array}{l}4150.82 \\
4150.627 \\
4147.37 \\
4146.874 \\
4141.454\end{array}$ & $\begin{array}{l}5 l, 7 \\
2 \\
1 \\
87 \\
107\end{array}$ & $\begin{array}{l}24084.83 \\
24085.95 \\
24104.87 \\
24107.75 \\
24139.30\end{array}$ & $\begin{array}{c}d^{2} \mathrm{G}_{41 / 2}-x^{4} \mathrm{G}_{41 / 2}^{0} \\
b^{2} \mathrm{I}_{51 / 2}-z^{4} \mathrm{H}_{61 / 2}^{\circ} \\
b^{4} \mathrm{P}_{11 / 2}-z^{4} \mathrm{~F}_{21 / 2}^{0} \\
a^{2} \mathrm{P}_{11 / 2}-z^{2} \mathrm{~S}_{01 / 2}^{0} \\
b^{4} \mathrm{D}_{31 / 2}-z^{6} \mathrm{P}_{31 / 2}^{0}\end{array}$ & $\begin{array}{l}4002.965 \\
4002.38 \\
4000.717 \\
\text { 3999. } 840 \\
3998.565 \\
\text { 3996. } 64\end{array}$ & $\begin{array}{l}47 \\
1 \\
3 \\
107 \\
107 \\
\\
2\end{array}$ & $\begin{array}{l}24974.42 \\
24978.07 \\
24988.45 \\
24993.93 \\
25001.90 \\
\\
25013.94\end{array}$ & $\begin{array}{l}d^{2} \mathrm{D}_{21 / 2}-w^{4} \mathrm{D}_{11 / 2}^{\circ} \\
b^{4} \mathrm{G}_{21 / 2}-z^{4} \mathrm{D}_{11 / 2}{ }^{\circ} \\
c^{2} \mathrm{G}_{31 / 2}-y{ }^{4} \mathrm{P}_{21 / 2}^{0} \\
c^{4} \mathrm{D}_{11 / 2}-z^{2} \mathrm{~S}_{01 / 3}^{\circ}\end{array}$ \\
\hline $\begin{array}{l}\text { 4139. } 780 \\
4138.733 \\
4136.761 \\
4136.49 \\
4130.85\end{array}$ & $\begin{array}{l}87 \\
2 \\
3 \\
2 s \\
3\end{array}$ & $\begin{array}{l}24149.06 \\
24155.17 \\
24166.68 \\
24168.27 \\
24201.26\end{array}$ & $\begin{array}{l}c^{2} \mathrm{D}_{11 / 2}-y{ }^{4} \mathrm{~F}_{21 / 2}^{\circ} \\
a^{4} \mathrm{~F}_{41 / 2}-z^{6} \mathrm{~F}_{41 / 2}^{\circ} \\
b^{4} \mathrm{D}_{11 / 2}-z^{4} \mathrm{P}_{21 / 2}^{\circ}\end{array}$ & $\begin{array}{l}3992.54 \\
3990.79 \\
\text { 3989. } 45 \\
3986.155\end{array}$ & $\begin{array}{c}1 \\
15 l, 7 \\
5 H \\
507\end{array}$ & $\begin{array}{l}25039.63 \\
25050.61 \\
25059.02 \\
25079.74\end{array}$ & 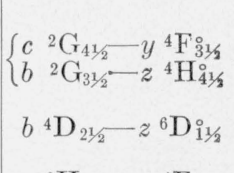 \\
\hline $\begin{array}{l}\text { 4130. } 28 \\
\text { 4127. } 296 \\
\text { 4126. } 31 \\
\text { 4125. } 628 \\
\text { 4123. } 26\end{array}$ & $\begin{array}{c}2 \\
2 \\
2 s \\
157 \\
1\end{array}$ & $\begin{array}{l}24204.61 \\
24222.10 \\
24227.89 \\
24231.90 \\
24245.81\end{array}$ & $\begin{array}{l}a^{2} \mathrm{~F}_{21 / 2}-z^{6} \mathrm{P}_{11 / 2}^{\circ} \\
d^{2} \mathrm{G}_{31 / 2}-x^{4} \mathrm{G}_{41 / 2}^{\circ} \\
c^{2} \mathrm{~F}_{31 / 2}-z^{2} \mathrm{G}_{41 / 2}^{\circ} \\
c^{4} \mathrm{D}_{01 / 2}-y^{4} \mathrm{D}_{11 / 2}^{\circ}\end{array}$ & $\begin{array}{l}3980.03 \\
3974.84 \\
3974.46 \\
3972.950 \\
3972.17\end{array}$ & $\begin{array}{l}5 H \\
4 h \\
1 \\
77 \\
2 h\end{array}$ & $\begin{array}{l}25118.33 \\
25151.13 \\
25153.53 \\
25163.10 \\
25168.04\end{array}$ & 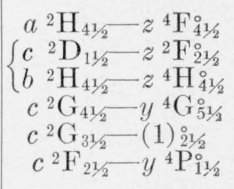 \\
\hline $\begin{array}{l}4122.350 \\
4119.634 \\
4118.53 \\
4117.58 \\
4115.76\end{array}$ & $\begin{array}{l}507 \\
407 \\
57 \\
3 \\
27\end{array}$ & $\begin{array}{l}24251.19 \\
24267.18 \\
24273.68 \\
24279.28 \\
24290.02\end{array}$ & $\begin{array}{l}c^{2} \mathrm{~F}_{31 / 2}-z^{2} \mathrm{D}_{21 / 2}^{\circ} \\
b^{4} \mathrm{D}_{31 / 2}-z^{6} \mathrm{P}_{22^{1}}^{\circ} \\
b^{2} \mathrm{I}_{51 / 2}-z^{4} \mathrm{~J} \\
c^{2} \mathrm{D}_{21 / 2}-x^{4} \mathrm{~F}_{31 / 2}^{\circ}\end{array}$ & $\begin{array}{l}\text { 3968. } 667 \\
3967.80 \\
3967.686 \\
3965.45 \\
3961.520\end{array}$ & $\begin{array}{c}207 \\
87 \\
3 \\
1 \\
2007\end{array}$ & $\begin{array}{l}25190.25 \\
25195.75 \\
25196.48 \\
25210.68 \\
25235.70\end{array}$ & $\begin{array}{c}b^{4} \mathrm{D}_{21 / 2}-z^{6} \mathrm{D}_{31 / 2}^{\circ} \\
a^{2} \mathrm{P}_{01 / 2}-y^{4} \mathrm{D}_{01 / 2}^{01 / 2} \\
b^{4} \mathrm{G}_{31 / 2}-z^{4} \mathrm{D}_{21 / 2}^{\circ} \\
b^{4} \mathrm{D}_{31 / 2}-z{ }^{6} \mathrm{D}_{21 / 2}^{\circ}\end{array}$ \\
\hline
\end{tabular}


TABLE 1. Wavelengths and term combinations of Mo II-Continued

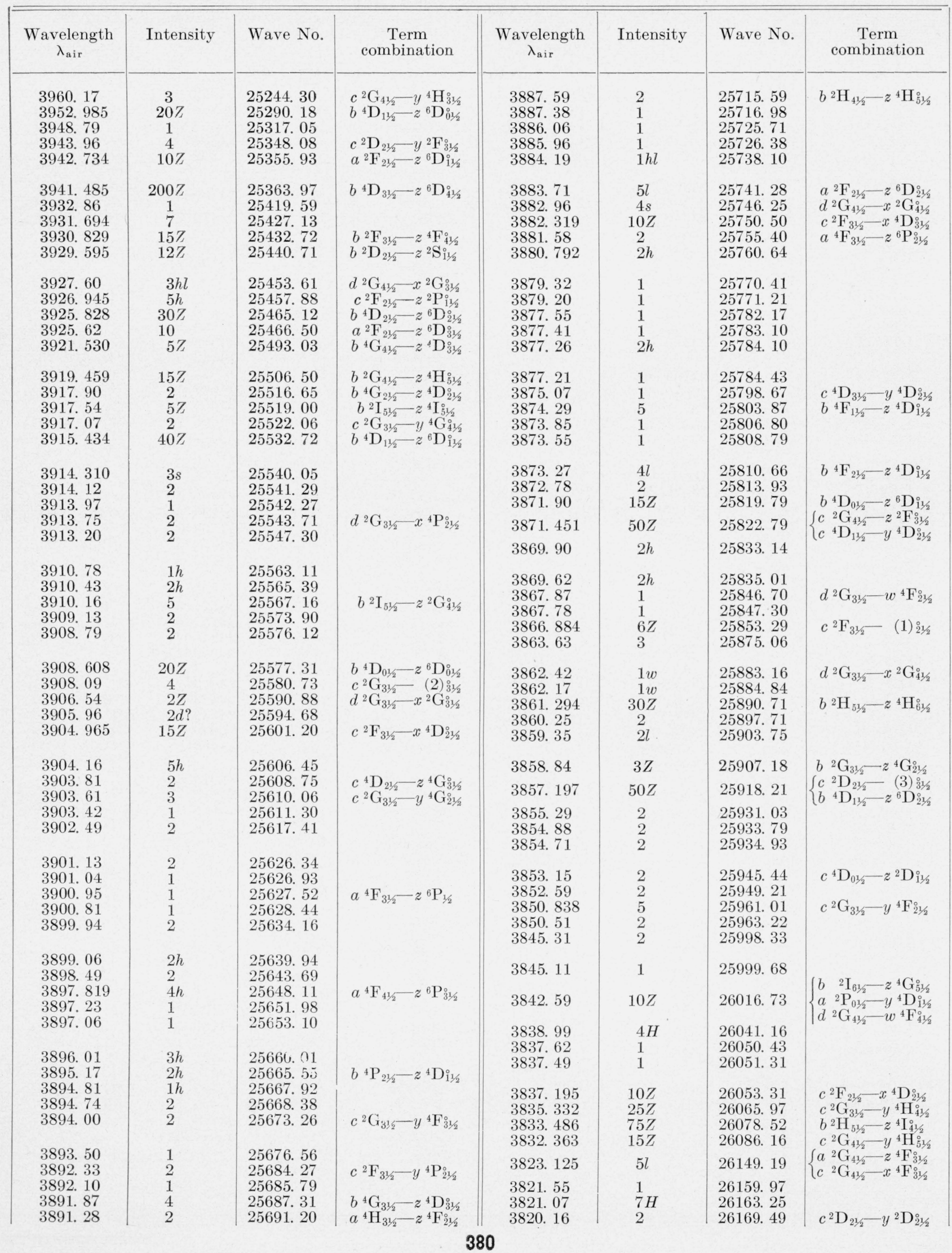


TABLE 1. Wavelengths and term combinations of Mo II-Continued

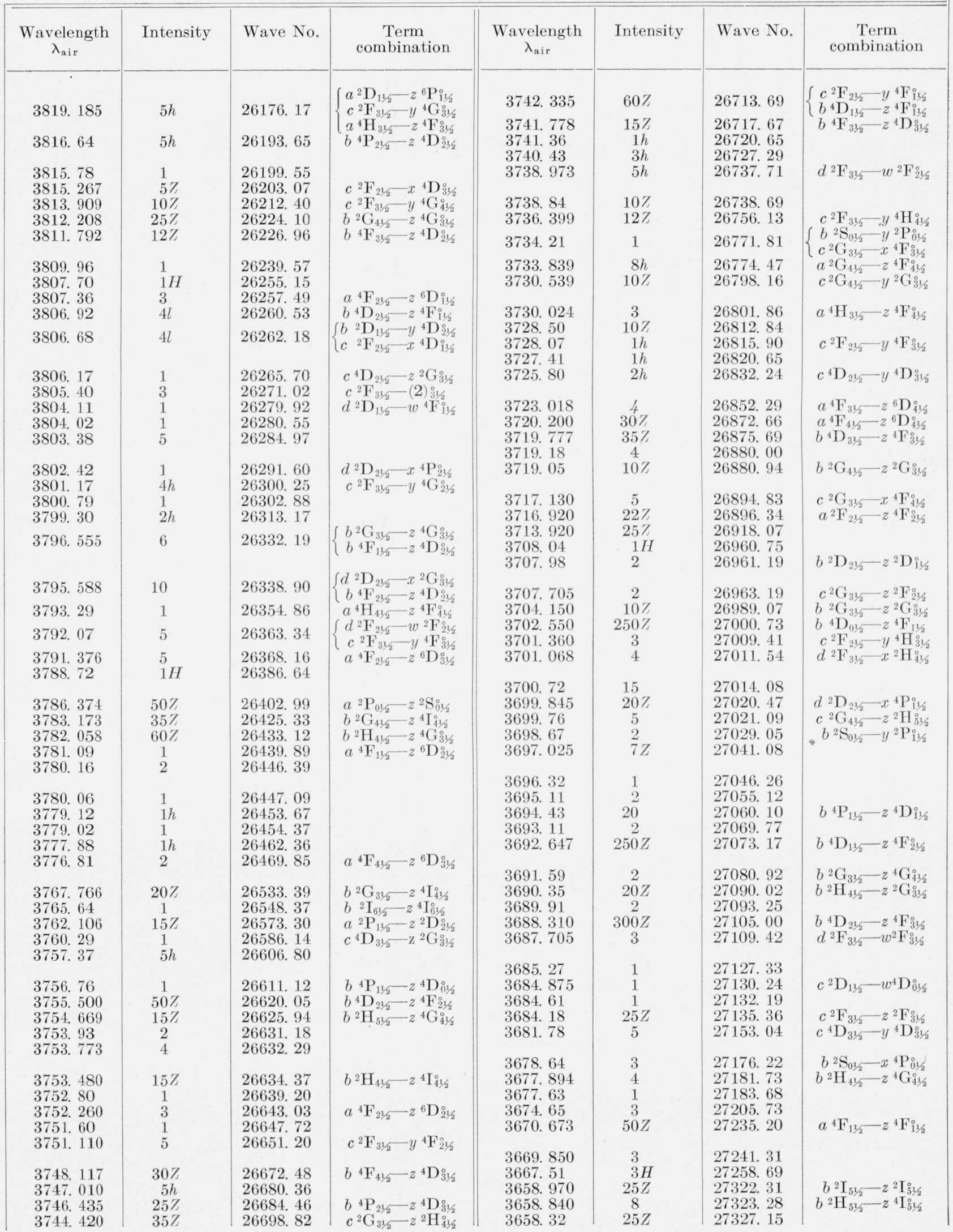


TABLE 1. Wavelengths and term combinations of Mo II-Continued

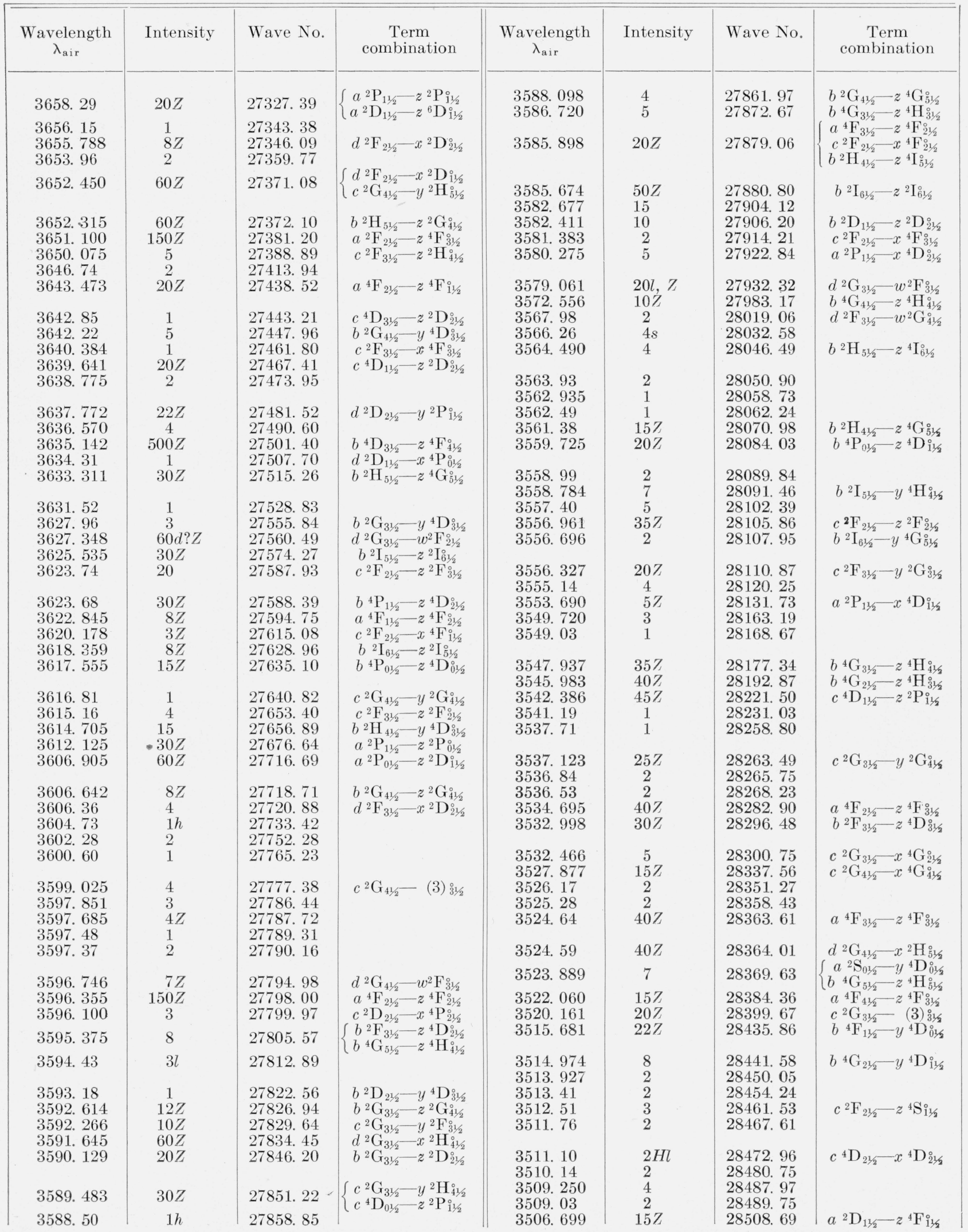


TABLE 1. Wavelengths and term combinations of Mo II-Continued

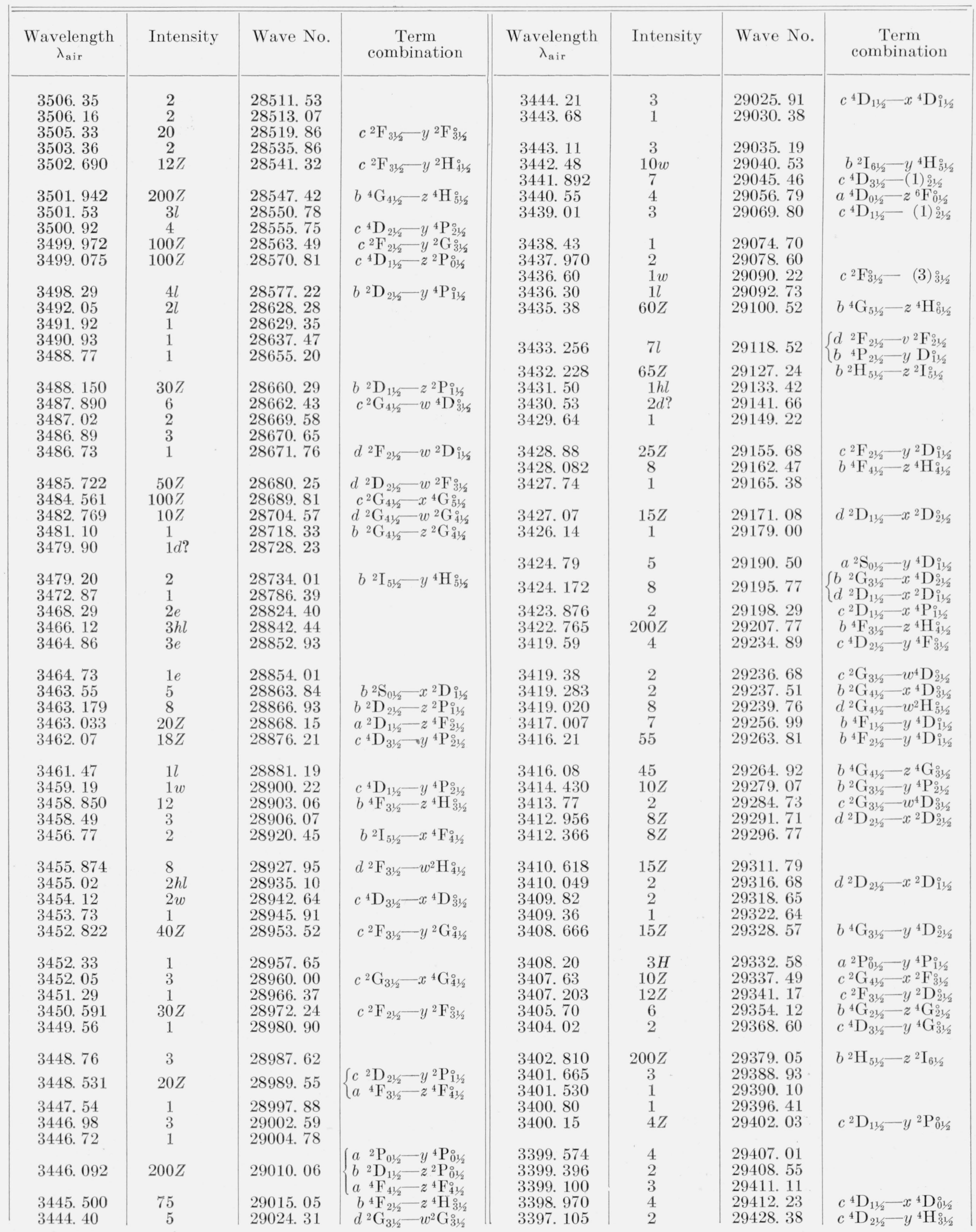


TABLE 1. Wavelengths and term combinations of Mo II-Continued

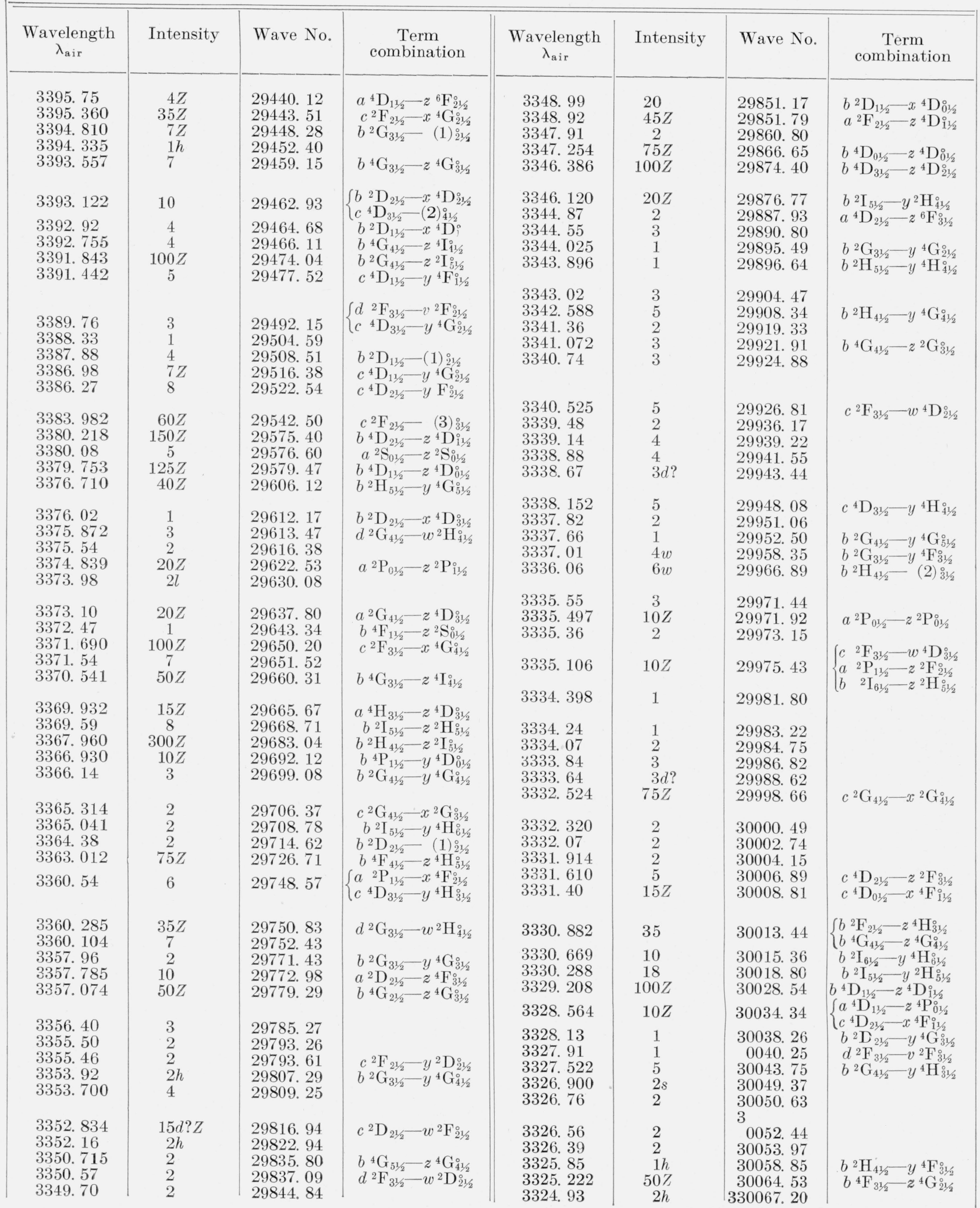


TABLE 1. Wavelengths and term combinations of Mo II-Continued

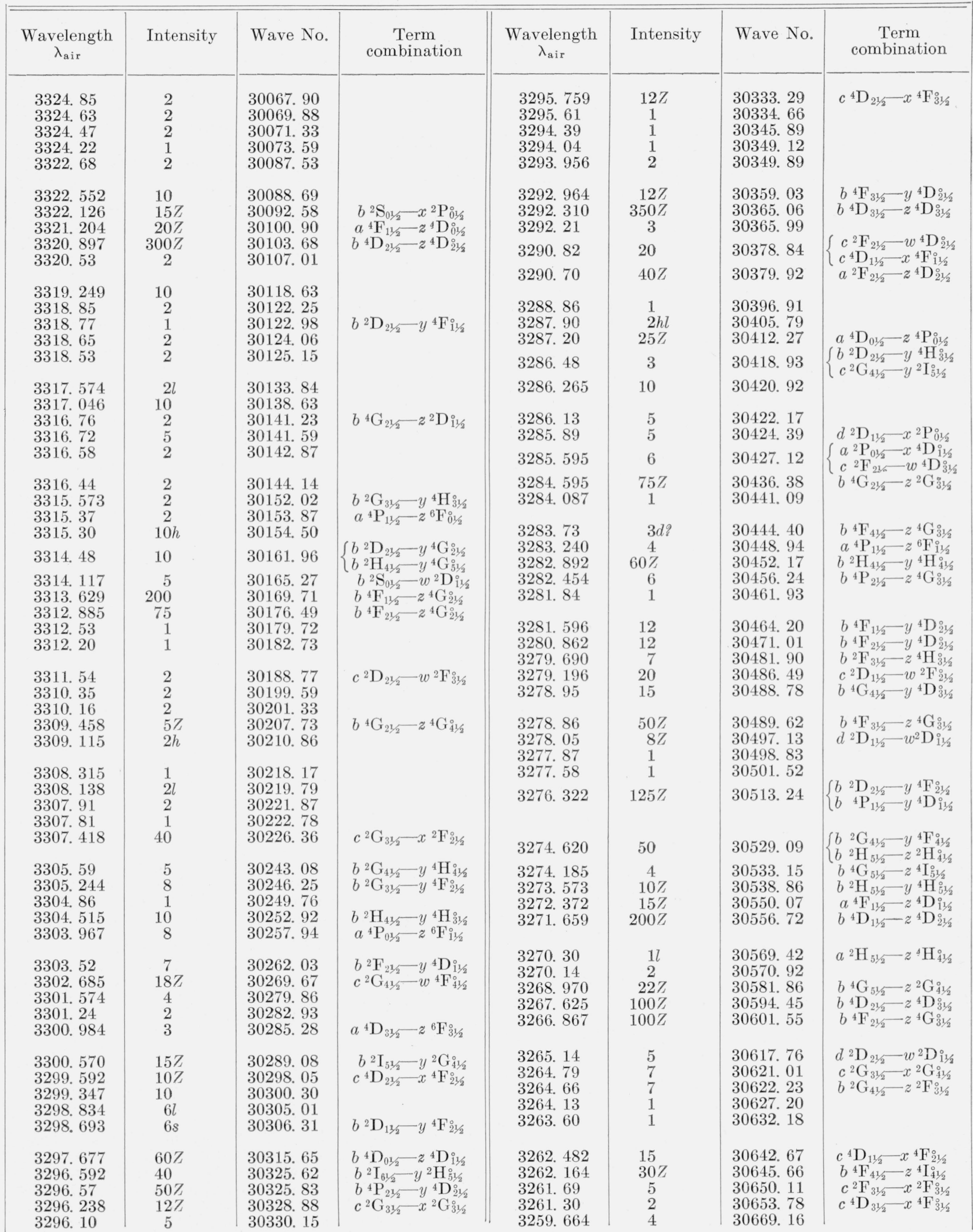


TABLE 1. Wavelengths and term combinations of Mo II-Continued

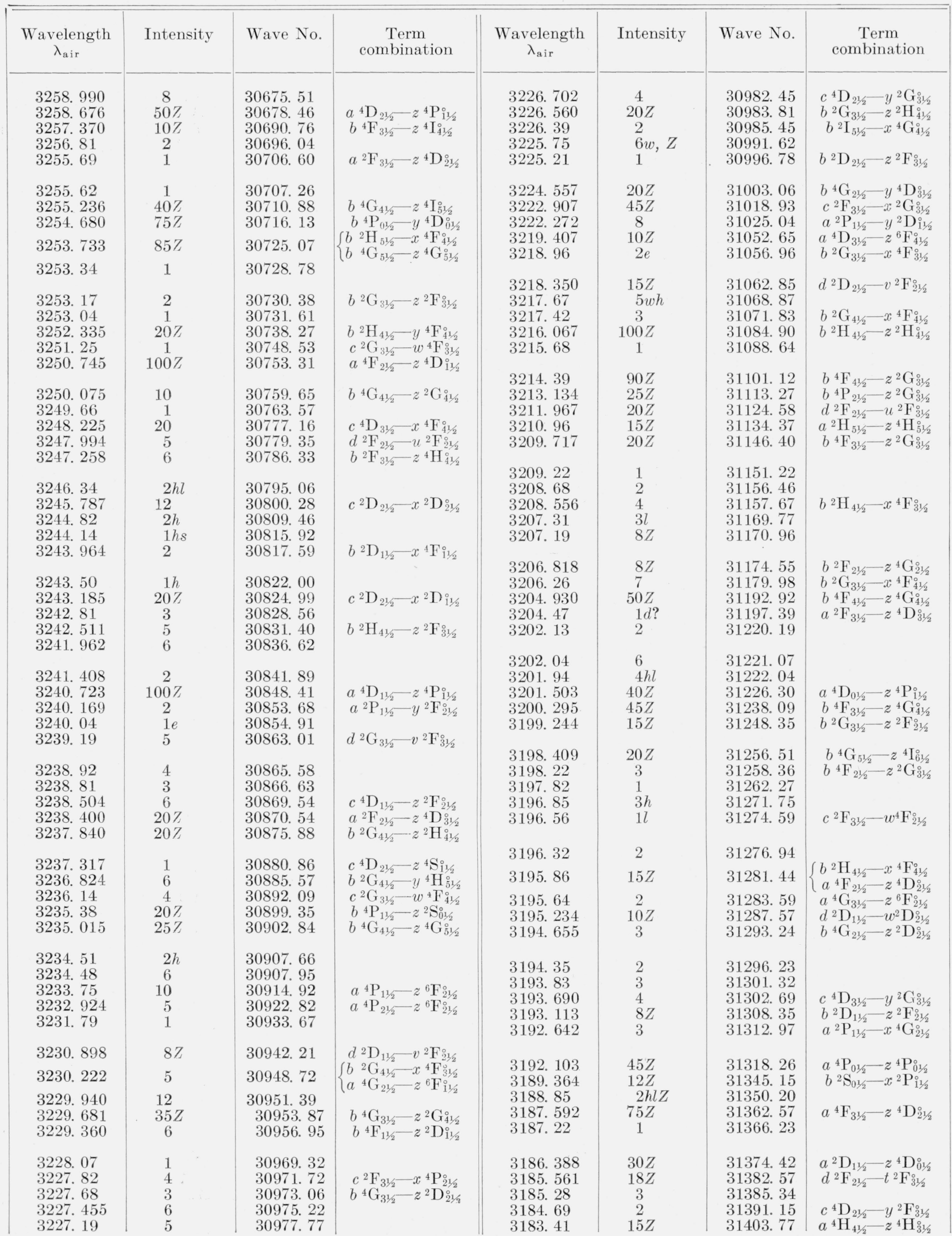


TABLE 1. Wavelengths and term combinations of Mo II-Continued

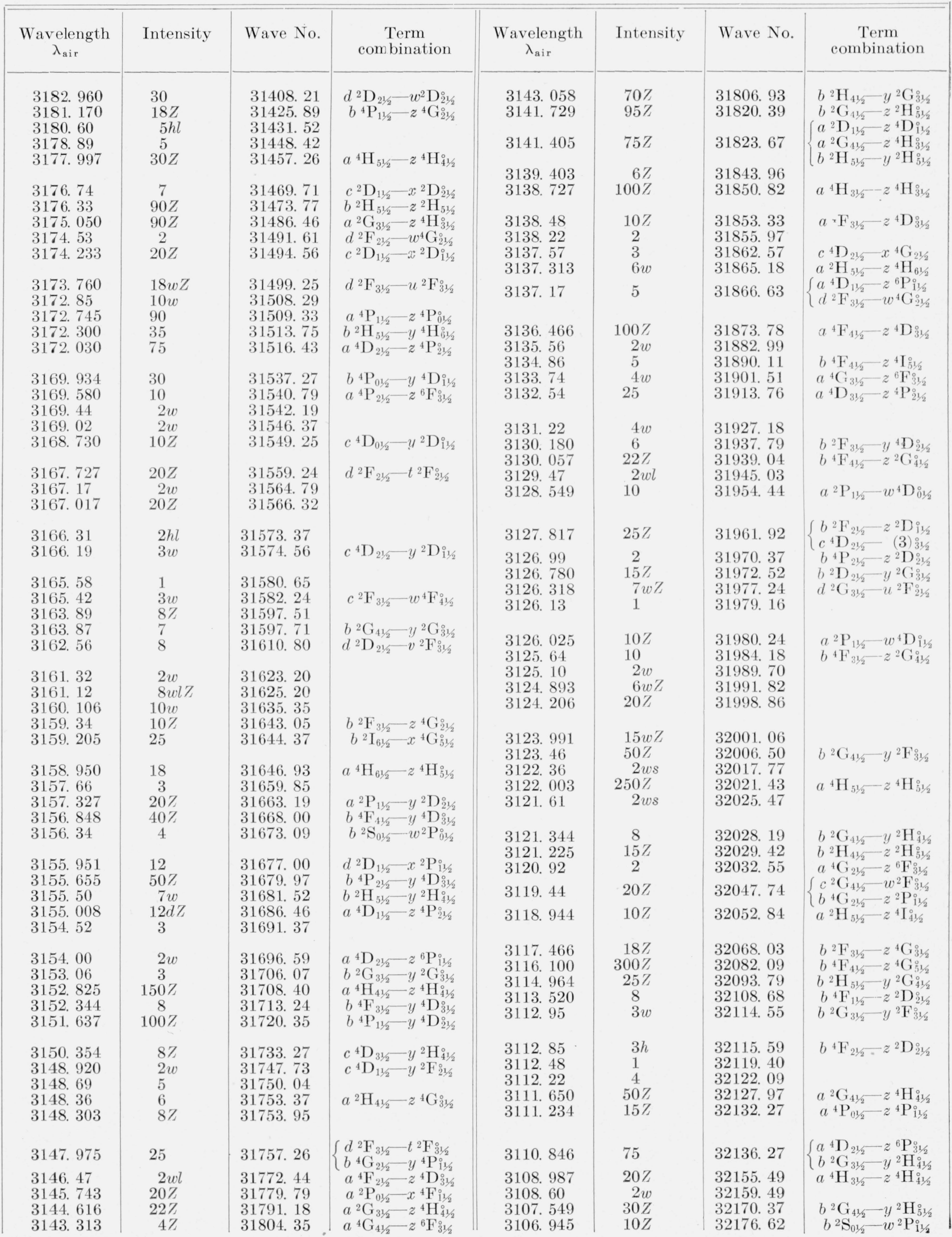


TABLE 1. Wavelengths and term combinations of Mo II-Continued

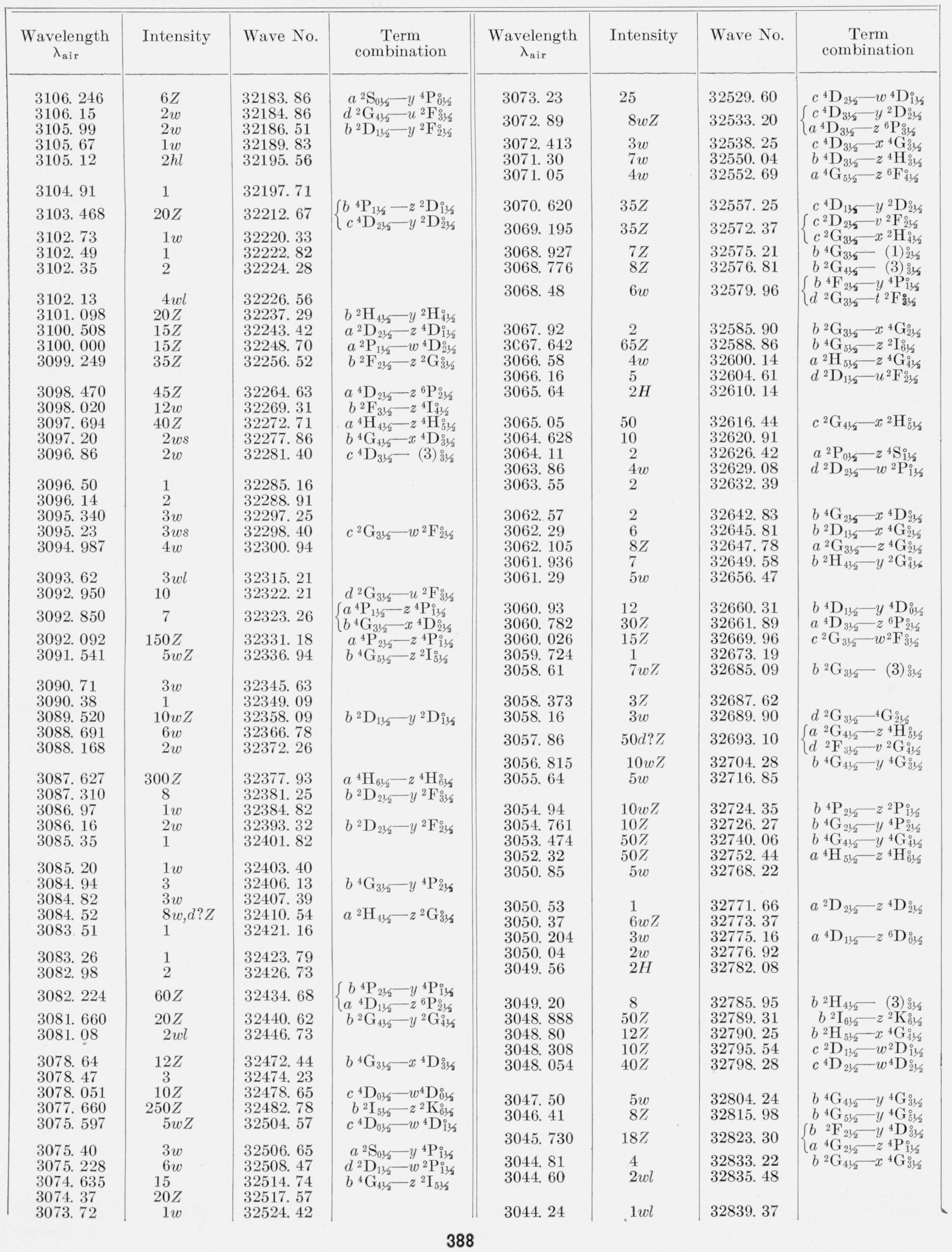


TABLE 1. Wavelengths and term combinations of Mo II-Continued

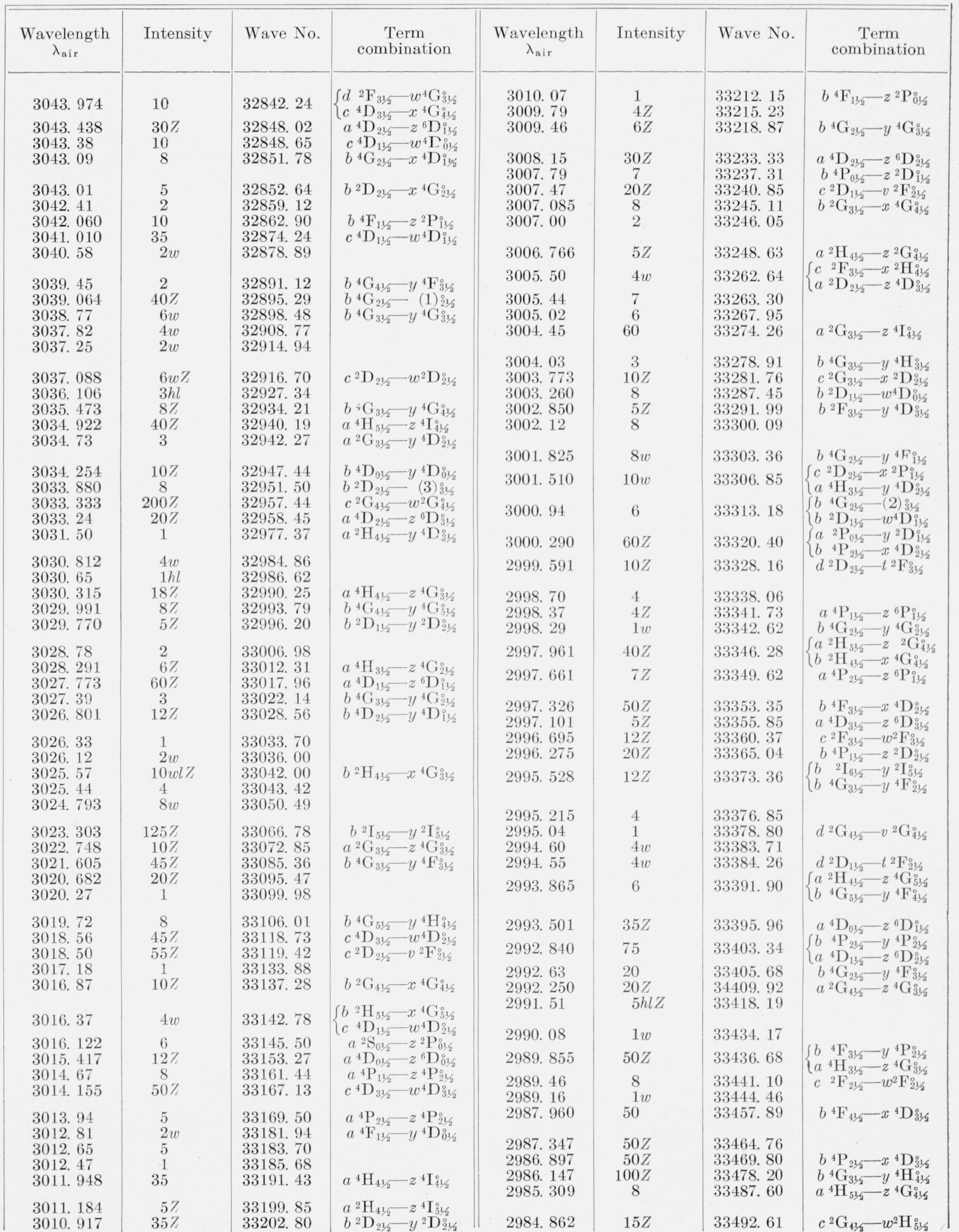


TABLE 1. Wavelengths and term combinations of Mo II-Continued

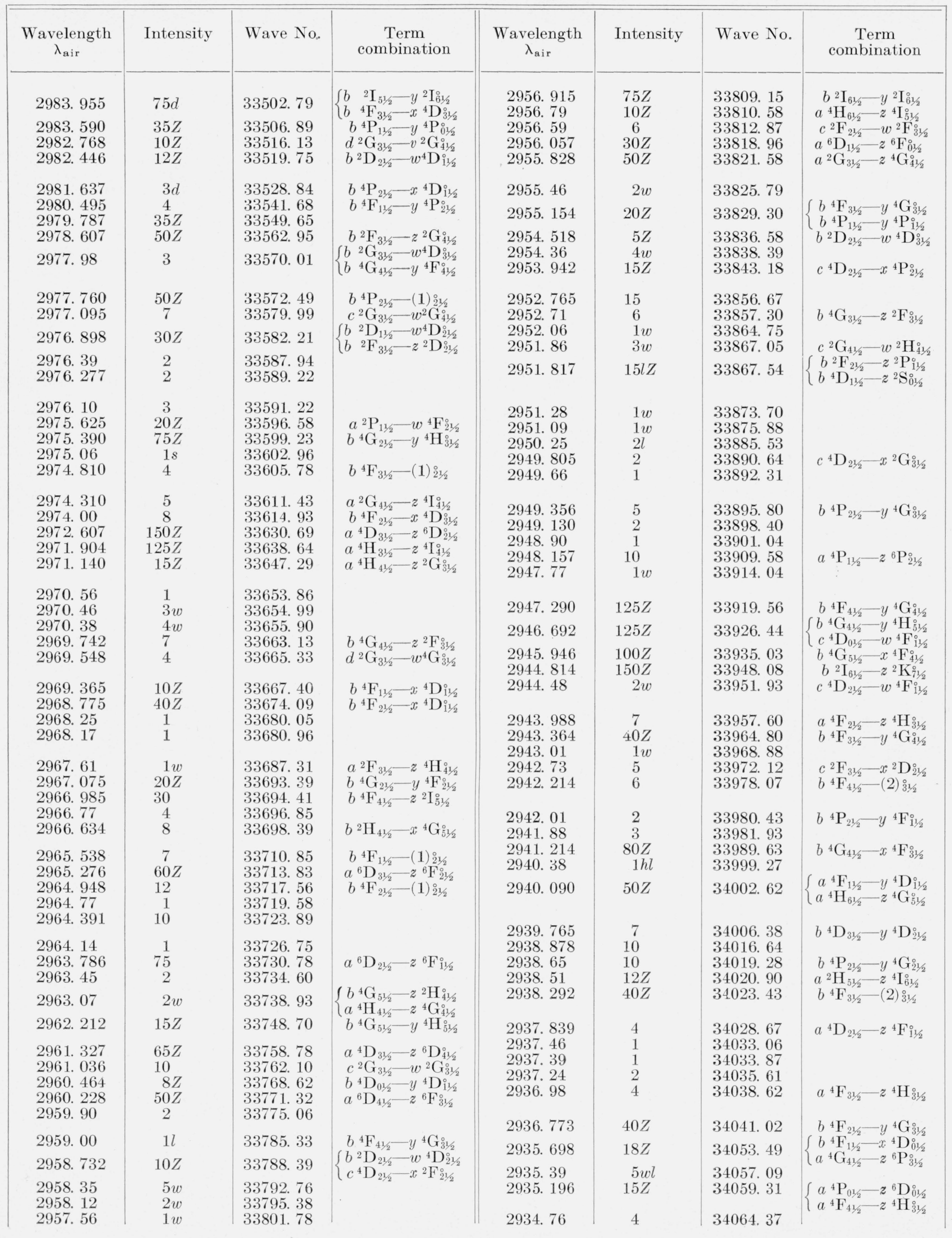


TABLE 1. Wavelengths and term combinations of Mo II-Continued

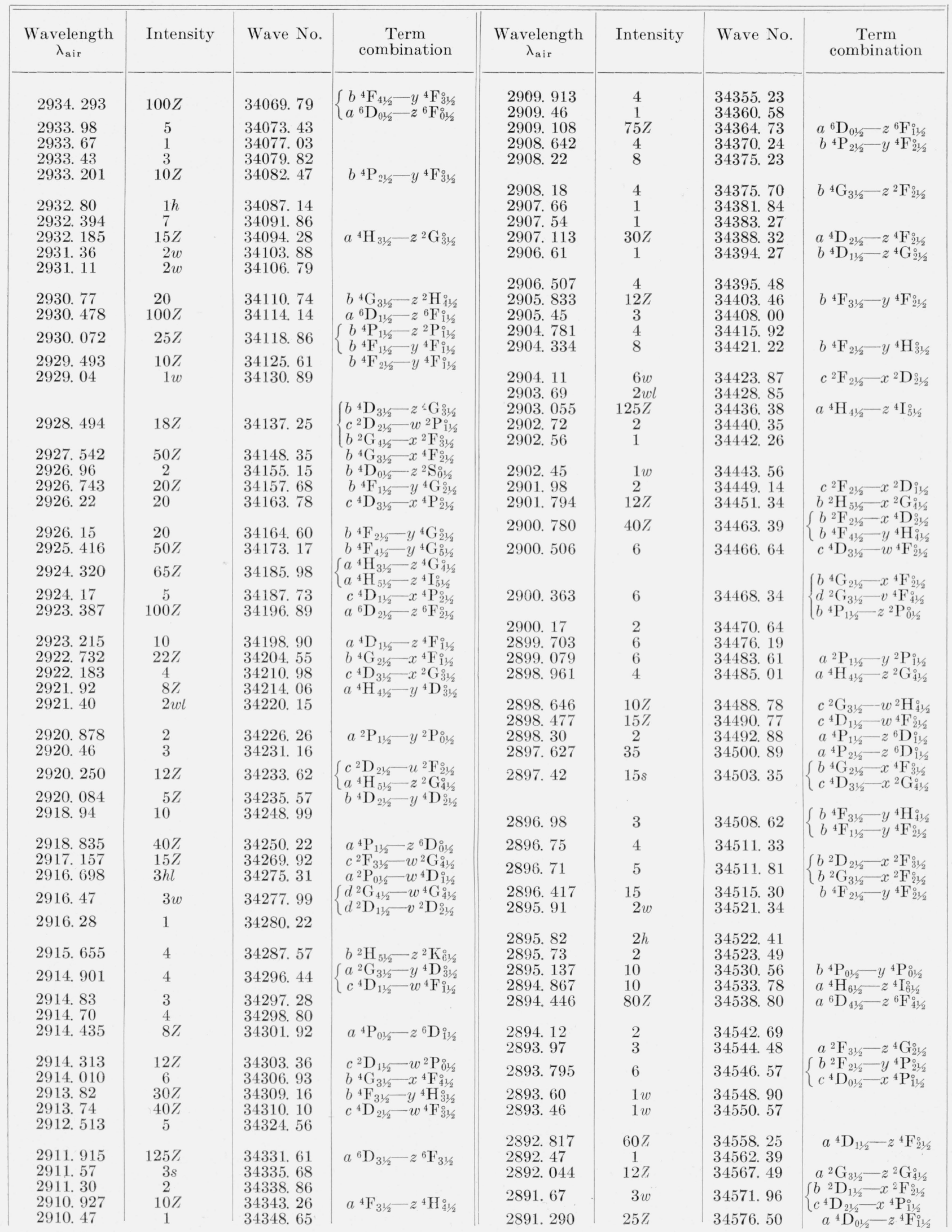


TABLE 1. Wavelengths and term combinations of Mo II-Continued

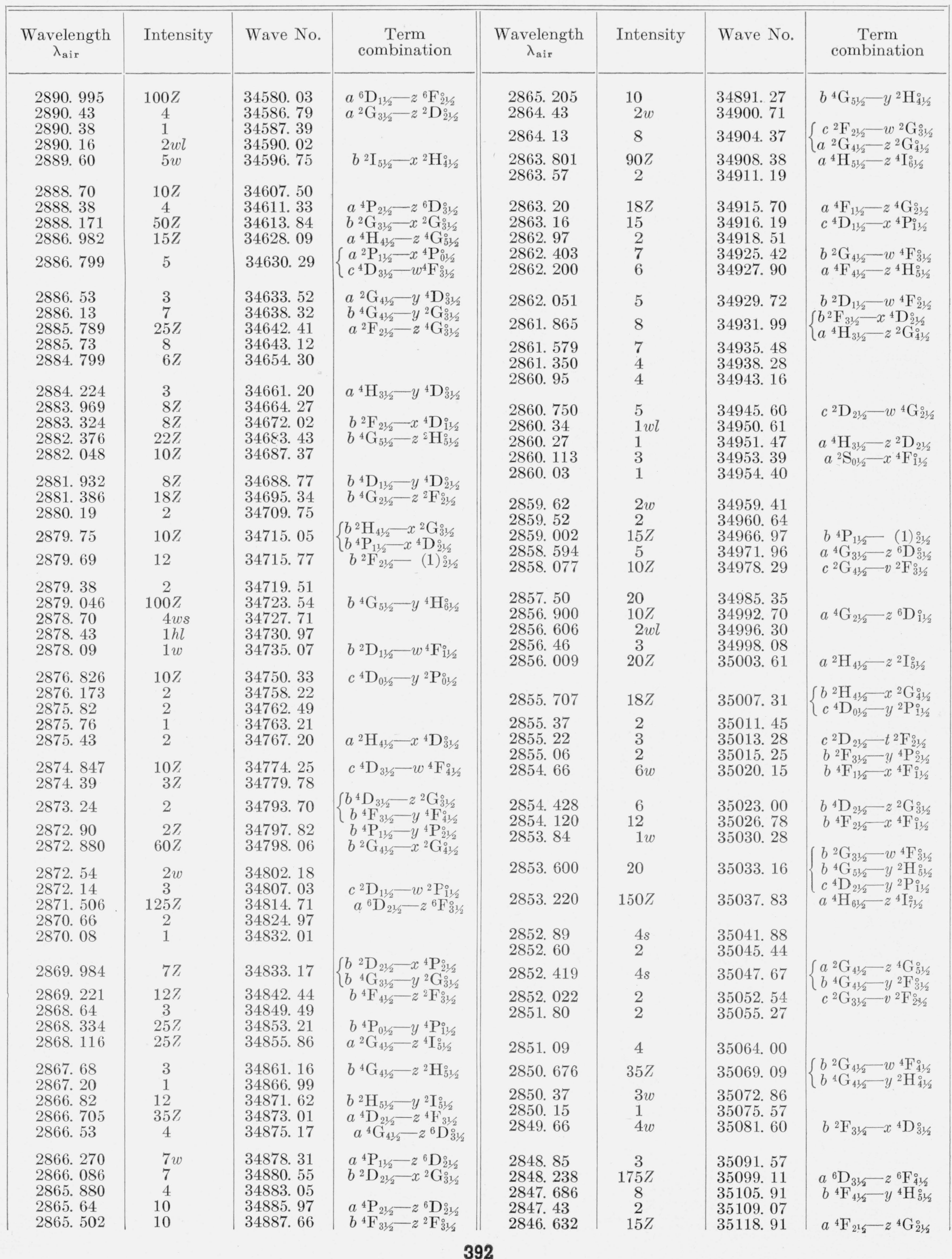


TABLE 1. Wavelengths and term combinations of Mo II-Continued

\begin{tabular}{|c|c|c|c|c|c|c|c|}
\hline $\begin{array}{c}\text { Wavelength } \\
\lambda_{\mathrm{air}}\end{array}$ & Intensity & Wave No. & $\begin{array}{c}\text { Term } \\
\text { combination }\end{array}$ & $\begin{array}{c}\text { Wavelength } \\
\lambda_{\mathrm{air}}\end{array}$ & Intensity & Wave No. & $\begin{array}{c}\text { Term } \\
\text { combination }\end{array}$ \\
\hline $\begin{array}{l}2846.250 \\
2846.08 \\
2845.668 \\
2845.392 \\
2845.222\end{array}$ & $\begin{array}{l}47 \\
1 \\
10 Z \\
6 \\
2\end{array}$ & $\begin{array}{l}\text { 35123. } 63 \\
35125.72 \\
35130.81 \\
35134.21 \\
35136.31\end{array}$ & $\begin{array}{l}b^{2} \mathrm{~F}_{21 / 2}-y^{4} \mathrm{~F}_{11 / 2}^{\circ} \\
b^{2} \mathrm{H}_{41 / 2}-w^{4} \mathrm{~F}_{31 / 2} \\
b^{2} \mathrm{D}_{21 / 2}-w^{4} \mathrm{~F}_{21 / 2}\end{array}$ & $\begin{array}{l}2822.035 \\
2821.843 \\
2821.02 \\
2820.38 \\
2820.22\end{array}$ & $\begin{array}{l}127 \\
18 Z \\
10 w l Z \\
3 w \\
2 h\end{array}$ & $\begin{array}{l}\text { 35424. } 99 \\
35427.40 \\
35437.74 \\
35445.78 \\
35447.79\end{array}$ & $\begin{array}{l}a^{6} \mathrm{D}_{01 / 2}-z^{4} \mathrm{P}_{01 / 2}^{\circ} \\
b^{2} \mathrm{H}_{41 / 2}^{0}-y^{2} \mathrm{I}_{51 / 2}^{\circ} \\
a^{2} \mathrm{D}_{21 / 2}-z{ }^{4} \mathrm{H}_{31 / 2}^{\circ}\end{array}$ \\
\hline $\begin{array}{l}2845.14 \\
2844.834 \\
2844.67 \\
2844.507 \\
2843.97\end{array}$ & $\begin{array}{l}2 \\
18 Z \\
1 \\
3 w \\
2\end{array}$ & $\begin{array}{l}35137.33 \\
35141.11 \\
35143.13 \\
35145.15 \\
35151.78\end{array}$ & $\begin{array}{l}b{ }^{4} \mathrm{~F}_{31 / 2}-z^{2} \mathrm{H}_{41 / 2}{ }^{\circ} \\
b^{4} \mathrm{P}_{01 / 2}-z^{2} \mathrm{P}_{11 / 2}^{11} \\
b^{4} \mathrm{P}_{21 / 2}-x^{4} \mathrm{~F}_{21 / 2}^{\circ}\end{array}$ & $\begin{array}{l}2820.018 \\
2819.594 \\
2818.98 \\
2818.81 \\
2818.56\end{array}$ & $\begin{array}{l}87 \\
8 Z \\
5 \\
2 \\
2 h l\end{array}$ & $\begin{array}{l}35450.33 \\
35455.66 \\
35463.38 \\
35465.52 \\
35468.67\end{array}$ & $b^{4} \mathrm{D}_{01 / 2}^{\circ}-z^{2} \mathrm{D}_{11 / 2}^{\circ}$ \\
\hline $\begin{array}{l}\text { 2843. } 734 \\
2843.09 \\
2842.492 \\
2842.148 \\
\text { 2841. } 788\end{array}$ & $\begin{array}{l}10 Z \\
4 \\
35 Z \\
40 Z \\
12 Z\end{array}$ & $\begin{array}{l}35154.70 \\
35162.66 \\
35170.06 \\
35174.31 \\
35178.77\end{array}$ & $\begin{array}{c}c^{4} \mathrm{D}_{01 / 2}-x^{4} \mathrm{P}_{01 / 2}^{\circ} \\
b^{2} \mathrm{~F}_{21 / 2}-y^{4} \mathrm{G}_{21 / 2}^{12} \\
a^{2} \mathrm{~F}_{31 / 2}-z^{4} \mathrm{I}_{41 / 2}^{0} \\
a^{6} \mathrm{D}_{11 / 2}-z^{4} \mathrm{P}_{01 / 2}^{0} \\
\left\{\begin{array}{l}c^{2} \mathrm{~F}_{31 / 2}-w^{2} \mathrm{H}_{41 / 2}^{0} \\
b^{4} \mathrm{~F}_{31 / 2}-x^{4} \mathrm{~F}_{21 / 2}^{0}\end{array}\right.\end{array}$ & $\begin{array}{l}2817.54 \\
2817.44 \\
2816.50 \\
2816.153 \\
2815.80\end{array}$ & $\begin{array}{r}15 Z \\
15 Z \\
4 \\
150 Z \\
4 w\end{array}$ & $\begin{array}{l}\text { 35481. } 51 \\
\text { 35482. } 77 \\
\text { 35494. } 61 \\
\text { 35498. } 98 \\
\text { 35503. } 43\end{array}$ & 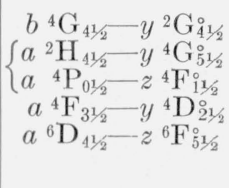 \\
\hline $\begin{array}{l}\text { 2841. } 60 \\
2841.56 \\
2841.34 \\
2841.11 \\
2840.080\end{array}$ & $\begin{array}{l}3 \\
1 \\
2 \\
2 \\
7 Z\end{array}$ & $\begin{array}{l}35181.10 \\
35181.59 \\
35184.32 \\
35187.16 \\
35199.93\end{array}$ & $\begin{array}{l}b{ }^{4} \mathrm{P}_{21 / 2}-x^{4} \mathrm{~F}_{31 / 2}^{0} \\
b^{4} \mathrm{D}_{11 / 2}-z^{2} \mathrm{D}_{11 / 2}^{0} \\
b^{2} \mathrm{~F}_{31 / 2}{ }^{2}(1)_{21 / 2}^{0} \\
a^{4} \mathrm{~F}_{31 / 2}-z^{4} \mathrm{G}_{21 / 2}^{0}\end{array}$ & $\begin{array}{l}2815.46 \\
2814.997 \\
2814.678 \\
2814.123 \\
2813.34\end{array}$ & $\begin{array}{r}3 \\
37 \\
127 \\
87 \\
47\end{array}$ & $\begin{array}{l}35507.72 \\
35513.56 \\
35517.58 \\
35524.59 \\
35534.47\end{array}$ & $\begin{array}{l}b^{2} \mathrm{~F}_{31 / 2}-y{ }^{4} \mathrm{G}_{31 / 2}^{\circ} \\
b^{2} \mathrm{~F}_{21 / 2}-y^{4} \mathrm{~F}_{21 / 2}^{2} \\
b^{4} \mathrm{~F}_{21 / 2}-z{ }^{2} \mathrm{~F}_{21 / 2}^{2} \\
c^{4} \mathrm{D}_{11 / 2}-x^{4} \mathrm{P}_{01 / 2}^{0}\end{array}$ \\
\hline $\begin{array}{l}\text { 2839. } 872 \\
\text { 2839. } 25 \\
2839.19 \\
2838.94 \\
2838.00\end{array}$ & $\begin{array}{l}3 \\
817 \\
25 Z \\
2 \\
3\end{array}$ & $\begin{array}{l}35202.50 \\
35210.22 \\
35210.96 \\
35214.06 \\
35225.72\end{array}$ & $\begin{array}{l}a^{4} \mathrm{~F}_{11 / 2}-y{ }^{4} \mathrm{D}_{21 / 2}^{\circ} \\
b^{4} \mathrm{G}_{41 / 2}-y{ }^{2} \mathrm{H}_{41 / 2}^{0} \\
b^{4} \mathrm{~F}_{31 / 2}-x^{4} \mathrm{~F}_{31 / 2}^{0} \\
b^{2} \mathrm{~F}_{21 / 2}-y{ }^{4} \mathrm{~F}_{31 / 2}^{0}\end{array}$ & $\begin{array}{l}\text { 2813. } 191 \\
2812.65 \\
2812.612 \\
2812.04 \\
2811.90\end{array}$ & $\begin{array}{l}5 \\
4 Z \\
6 Z \\
1 \\
1\end{array}$ & $\begin{array}{ll}35536 . & 35 \\
35543 . & 21 \\
35543 . & 67 \\
35550 . & 90 \\
35552 . & 67\end{array}$ & $\begin{array}{l}b^{2} \mathrm{~F}_{31 / 2}-y^{4} \mathrm{G}_{41 / 2}^{\circ} \\
a^{4} \mathrm{~F}_{21 / 2} z^{4} \mathrm{G}_{31 / 2}^{\circ}\end{array}$ \\
\hline $\begin{array}{l}\text { 2836. } 721 \\
2836.307 \\
2835.342 \\
2834.993 \\
2834.873\end{array}$ & $\begin{array}{l}307 \\
107 \\
30 Z \\
10 \\
18\end{array}$ & $\begin{array}{l}35241.60 \\
35246.75 \\
35258.74 \\
35263.08 \\
35264.58 \\
35270.26\end{array}$ & 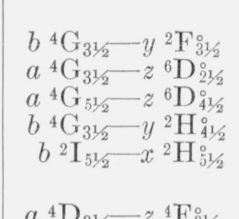 & $\begin{array}{l}\text { 2811. } 387 \\
2811.165 \\
2810.45 \\
2810.25 \\
2809.72\end{array}$ & $\begin{array}{l}37 \\
87 \\
37 \\
3 \\
2\end{array}$ & $\begin{array}{l}\text { 35559. } 16 \\
\text { 35561. } 97 \\
35571.01 \\
\text { 35573. } 54 \\
\text { 35580. } 25\end{array}$ & 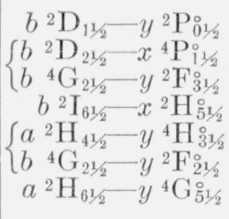 \\
\hline 2833. 796 & 8 & 35277.98 & $\left\{\begin{array}{l}b^{2} \mathrm{H}_{41 / 2}-w^{4} \mathrm{~F}_{41 / 2}^{0} \\
a^{4} \mathrm{G}_{41 / 2}-z^{6} \mathrm{D}^{4}{ }^{4} 1 / 2\end{array}\right.$ & $\begin{array}{l}\text { 2808. } 974 \\
2808.473\end{array}$ & $\begin{array}{r}10 Z \\
5 Z\end{array}$ & $\begin{array}{l}\text { 35589. } 70 \\
\text { 35596. } 05\end{array}$ & $b^{4} \mathrm{D}_{21 / 2}-y{ }^{4} \mathrm{D}_{31 / 2}^{0}$ \\
\hline $\begin{array}{l}2833.71 \\
2833.333 \\
2832.788\end{array}$ & $\begin{array}{l}2 w \\
6 \\
10\end{array}$ & $\begin{array}{l}\text { 35279. } 05 \\
35283.74 \\
35290.53\end{array}$ & $\begin{array}{l}b^{4} \mathrm{~F}_{11 / 2}-x^{4} \mathrm{~F}_{21 / 2}^{\circ} \\
b^{4} \mathrm{~F}_{21 / 2}-x^{4} \mathrm{~F}_{21 / 2}^{\circ}\end{array}$ & $\begin{array}{l}2808.09 \\
2807.750 \\
2807.016\end{array}$ & $\begin{array}{c}3 \\
100 Z \\
15 Z\end{array}$ & $\begin{array}{l}35600.91 \\
35605.22 \\
35614.53\end{array}$ & $\begin{array}{c}c^{2} \mathrm{G}_{31 / 2}-v^{2} \mathrm{~F}_{31 / 2}^{\circ} \\
\left\{\begin{array}{l}b{ }^{2} \mathrm{I}_{51 / 2}-w^{2} \mathrm{G}_{41 / 2}^{\circ} \\
a^{6} \mathrm{D}_{21 / 2}-z^{4} \mathrm{P}_{11 / 2}^{\circ}\end{array}\right. \\
a^{4} \mathrm{H}_{61 / 2} z^{2} \mathrm{I}_{51 / 2}^{\circ}\end{array}$ \\
\hline $\begin{array}{l}2832.663 \\
2832.078 \\
2832.00 \\
2831.76 \\
2831.442 \\
\\
2830.61\end{array}$ & $\begin{array}{l}10 \\
20 Z \\
10 Z \\
3 \\
40 Z \\
\\
3 w\end{array}$ & $\begin{array}{l}\text { 35292. } 09 \\
35299.38 \\
35300.35 \\
35303.34 \\
\text { 35307. } 31 \\
\text { 35317. } 68\end{array}$ & $\begin{array}{l}b^{4} \mathrm{~F}_{41 / 2}-x^{4} \mathrm{~F}_{41 / 2}^{0} \\
a^{2} \mathrm{~F}_{21 / 2}-z^{2} \mathrm{G}_{31 / 2}^{3} \\
b^{2} \mathrm{D}_{21 / 2}-w^{4} \mathrm{~F}_{31 / 2}^{\circ} \\
b^{4} \mathrm{G}_{51 / 2}-y^{2} \mathrm{G}_{41 / 2}^{0} \\
b^{2} \mathrm{H}_{51 / 2}-y^{2} \mathrm{I}_{61 / 2}^{\circ}\end{array}$ & $\begin{array}{ll}\text { 2806. } & 193 \\
2805 . & 70 \\
2804 . & 59 \\
2802 . & 360 \\
2801 . & 244\end{array}$ & $\begin{array}{r}8 Z \\
1 w \\
1 \\
15 \\
10\end{array}$ & $\begin{array}{l}\text { 35624. } 97 \\
\text { 35631. } 23 \\
\text { 35645. } 33 \\
\text { 35673. } 70 \\
\text { 35687. } 91\end{array}$ & 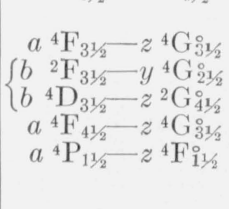 \\
\hline 2829. 88 & 4 & 35326. 79 & $\left\{\begin{array}{c}b^{4} \mathrm{~F}_{21 / 2}-x^{4} \mathrm{~F}_{31 / 2}^{\circ} \\
a^{2} \mathrm{H}_{51 / 2}-y^{4} \mathrm{G}_{41 / 2}^{0}\end{array}\right.$ & $\begin{array}{l}2800.745 \\
2800.363\end{array}$ & $\begin{array}{l}10 Z \\
10\end{array}$ & $\begin{array}{l}35694.27 \\
35699.13\end{array}$ & $b^{2} \mathbf{F}_{31 / 2}-y{ }^{4} \mathbf{F}_{31 / 2}^{\circ}$ \\
\hline $\begin{array}{l}\text { 2829. } 039 \\
2827.752 \\
2827.42\end{array}$ & $\begin{array}{c}12 Z \\
100 Z \\
2\end{array}$ & $\begin{array}{l}35337.29 \\
35353.38 \\
35357.53\end{array}$ & $\begin{array}{c}b^{4} \mathrm{~F}_{31 / 2}-x{ }^{4} \mathrm{~F}_{41 / 2}^{01 / 2} \\
a^{2} \mathrm{H}_{51 / 2}-z^{2} \mathrm{I}_{61 / 2}^{0} \\
d^{2} \mathrm{G}_{41 / 2}-w^{4} \mathrm{G}_{51 / 2}^{2}\end{array}$ & $\begin{array}{l}2800.505 \\
2800.057 \\
2798.910 \\
2798.070\end{array}$ & $\begin{array}{r}87 \\
107 \\
20 Z\end{array}$ & $\begin{array}{l}35699 . \\
35703.03 \\
35717.67 \\
35728.39\end{array}$ & $\begin{array}{l}a^{4} \mathrm{~F}_{11 / 2} z^{2} \mathrm{D}_{11 / 2}^{\circ}{ }^{11 / 2} \\
a^{2} \mathrm{~F}_{31 / 2} z^{4} \mathrm{G}_{41 / 2}^{\circ} \\
b^{4} \mathrm{P}_{21 / 2} z^{4} \mathrm{~S}_{11 / 2}^{\circ}\end{array}$ \\
\hline $\begin{array}{l}2827.185 \\
2826.550 \\
2826.017 \\
2825.95\end{array}$ & $\begin{array}{c}10 Z \\
10 \\
6 \\
4\end{array}$ & $\begin{array}{l}35360.47 \\
35368.41 \\
35375.08 \\
35375.92\end{array}$ & 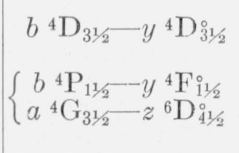 & $\begin{array}{l}2796.93 \\
2796.777 \\
2796.342 \\
2794.604 \\
2794.38\end{array}$ & $\begin{array}{l}5 e Z \\
8 Z \\
5 Z \\
5 Z \\
1\end{array}$ & $\begin{array}{l}35744.91 \\
35750.46 \\
35772.70 \\
35775.57\end{array}$ & 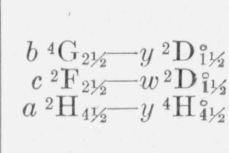 \\
\hline $\begin{array}{l}\text { 2825. } 60 \\
2825.299 \\
2824.188\end{array}$ & $\begin{array}{l}1 \\
8 Z \\
8 Z\end{array}$ & $\begin{array}{l}\text { 35377. } 80 \\
\\
\text { 35380. } 30 \\
\text { 35384. } 07 \\
\text { 35397. } 99\end{array}$ & 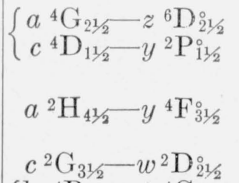 & $\begin{array}{l}2792.387 \\
2791.553 \\
2790.427 \\
2789.485 \\
2789.10\end{array}$ & $\begin{array}{l}5 w \\
507 \\
307 \\
2 \\
1\end{array}$ & $\begin{array}{l}\text { 35801. } 10 \\
\text { 35811. } 79 \\
\text { 35826. } 24 \\
\text { 35838. } 34 \\
\text { 35843. } 29\end{array}$ & $\begin{array}{l}b^{4} \mathrm{G}_{31 / 2}-(3)_{31 / 2}^{o} \\
a^{4} \mathrm{~F}_{31 / 2}-z^{4} \mathrm{I}_{41 / 2}^{01 / 2}\end{array}$ \\
\hline $\begin{array}{l}2822.937 \\
2822.416\end{array}$ & $\begin{array}{c}10 Z \\
7\end{array}$ & $\begin{array}{l}35413.68 \\
35420.21\end{array}$ & 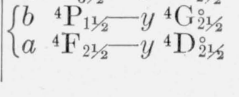 & $\begin{array}{l}2788.86 \\
2788.660 \\
2787.558\end{array}$ & $\begin{array}{l}5 \\
8 \\
1\end{array}$ & $\begin{array}{l}\text { 35846. } 37 \\
\text { 35848. } 94 \\
35863.12\end{array}$ & $\begin{array}{l}a^{4} \mathbf{F}_{41 / 2}-z^{4} \mathrm{I}_{41 / 2} \\
b^{4} \mathrm{~F}_{31 / 2}-y^{2} \mathrm{G}_{31 / 2}^{\circ}\end{array}$ \\
\hline
\end{tabular}


TABLE 1. Wavelengths and term combinations of Mo II-Continued

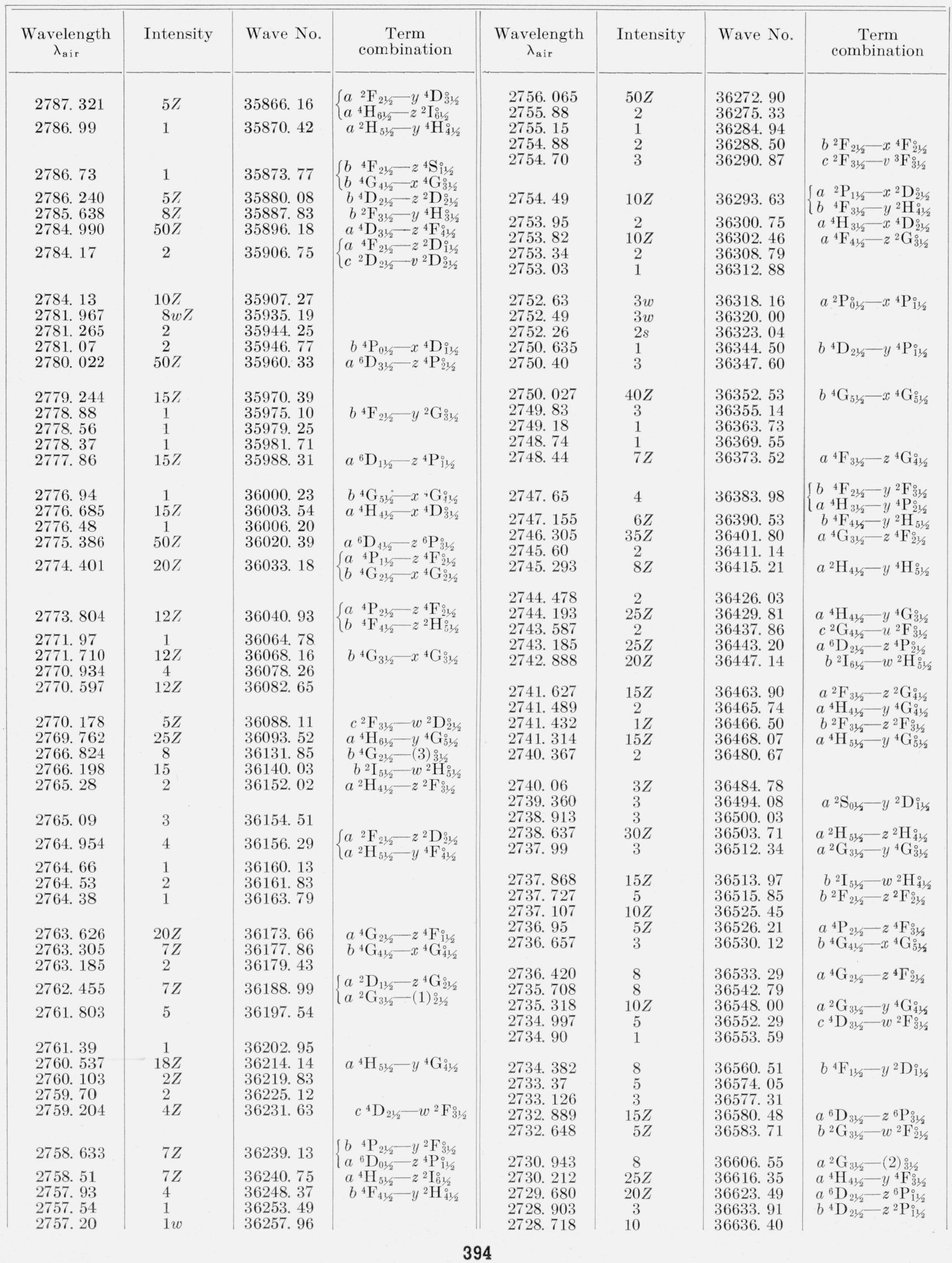


TABLE 1. Wavelengths and term combinations of Mo II-Continued

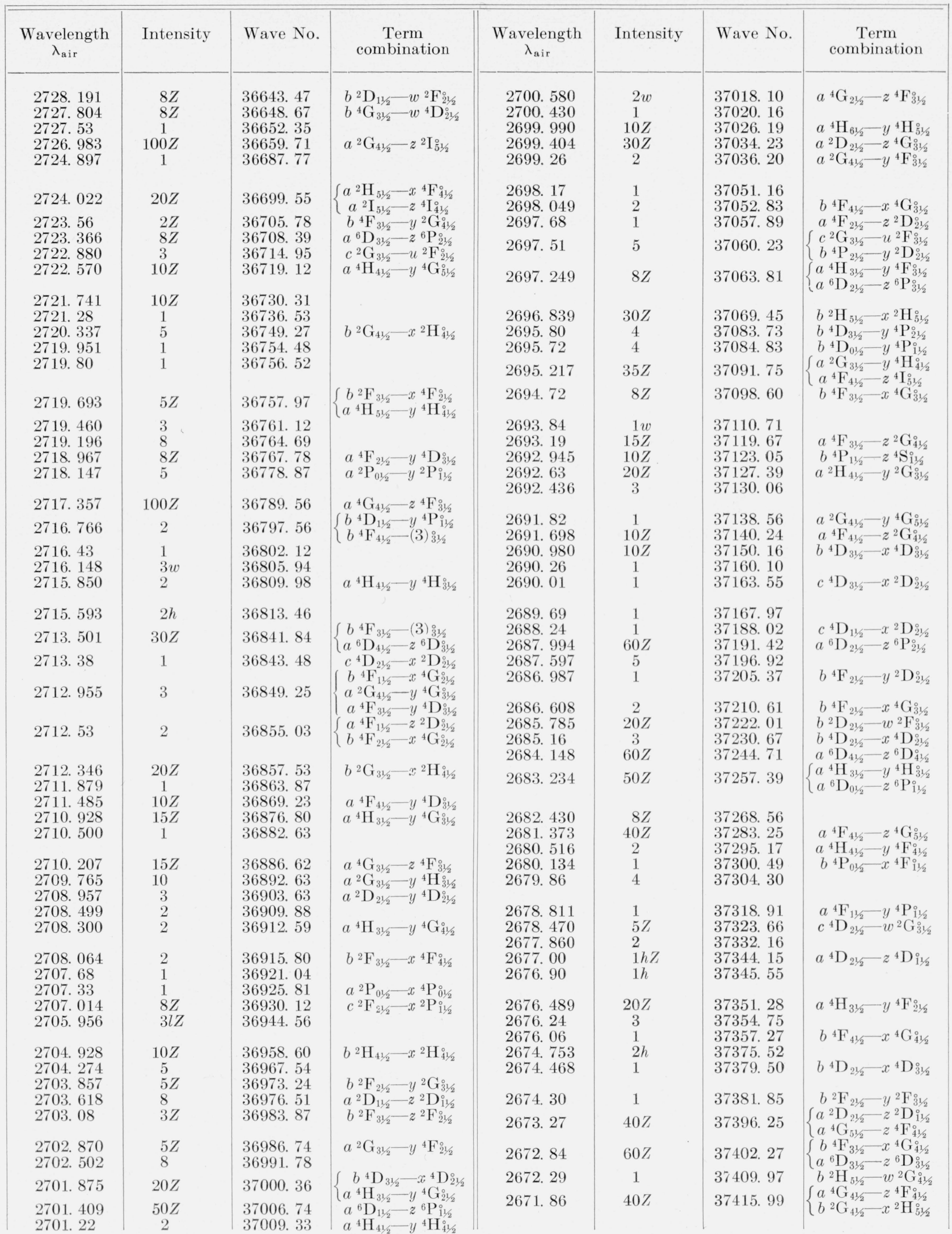


TABLE 1. Wavelengths and term combinations of Mo I-Continued

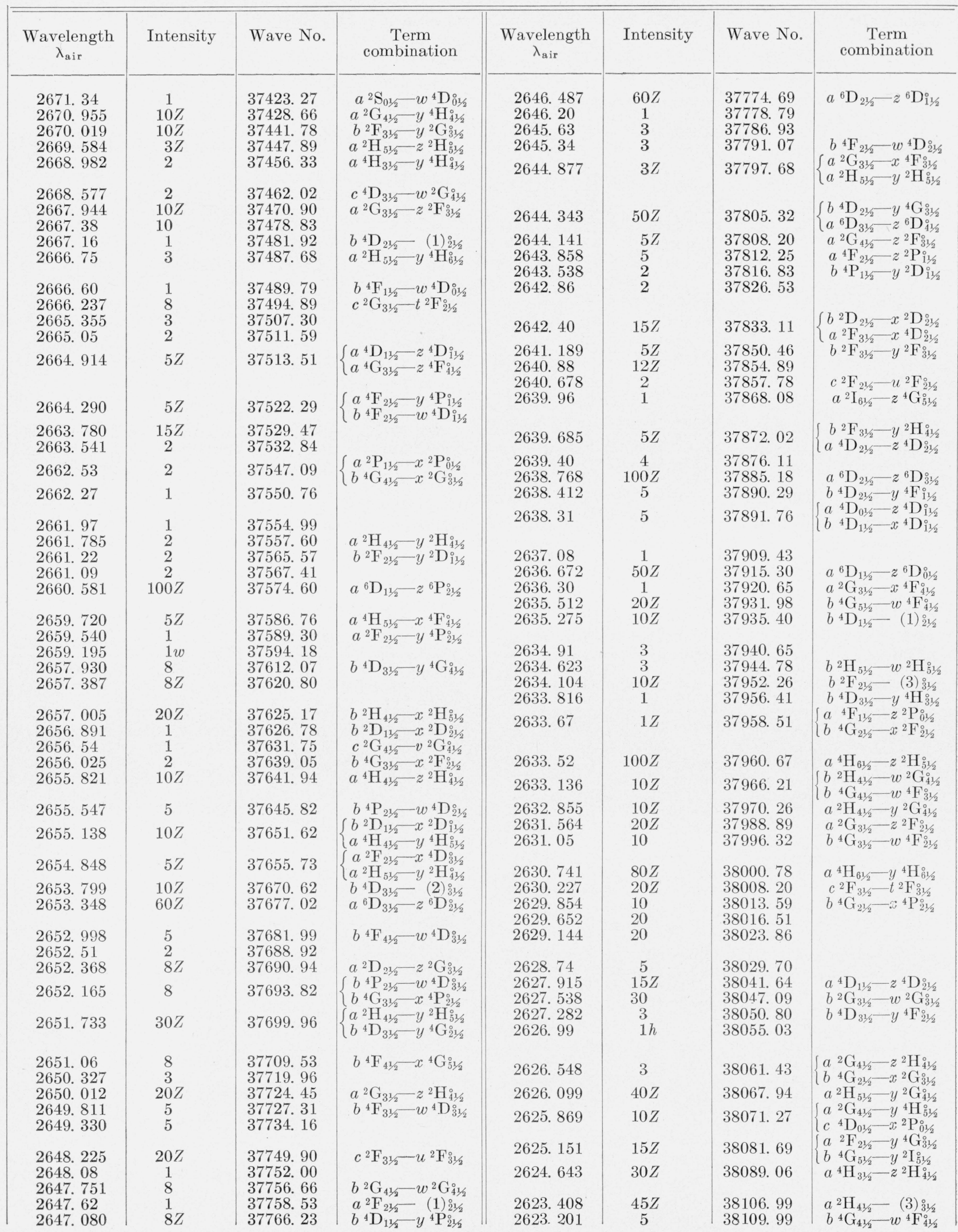


TABLE 1. Wavelengths and term combinations of Mo II-Continued

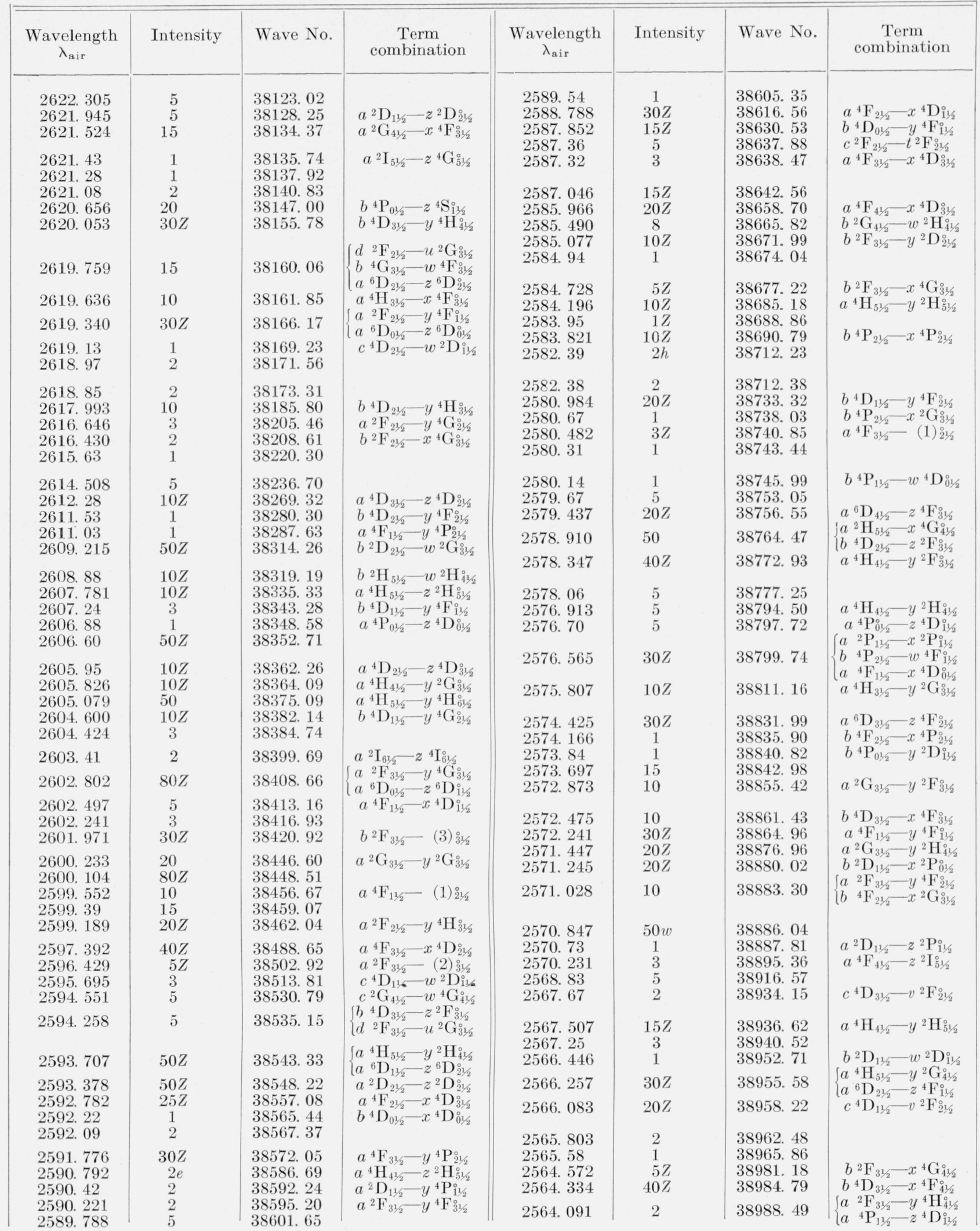


TABLE 1. Wavelengths and term combinations of Mo II-Continued

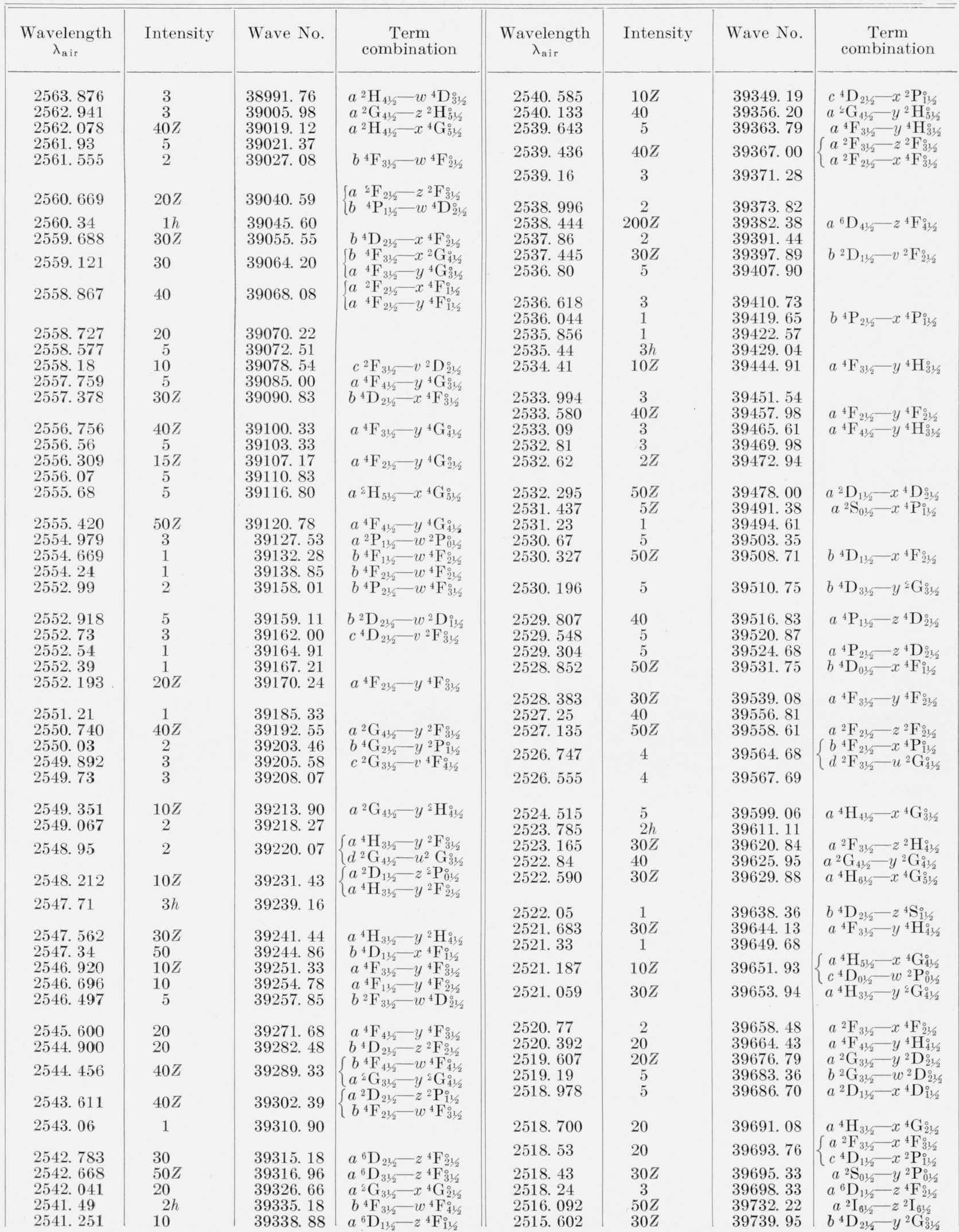


TABLE 1. Wavelengths and term combinations of Mo II-Continued

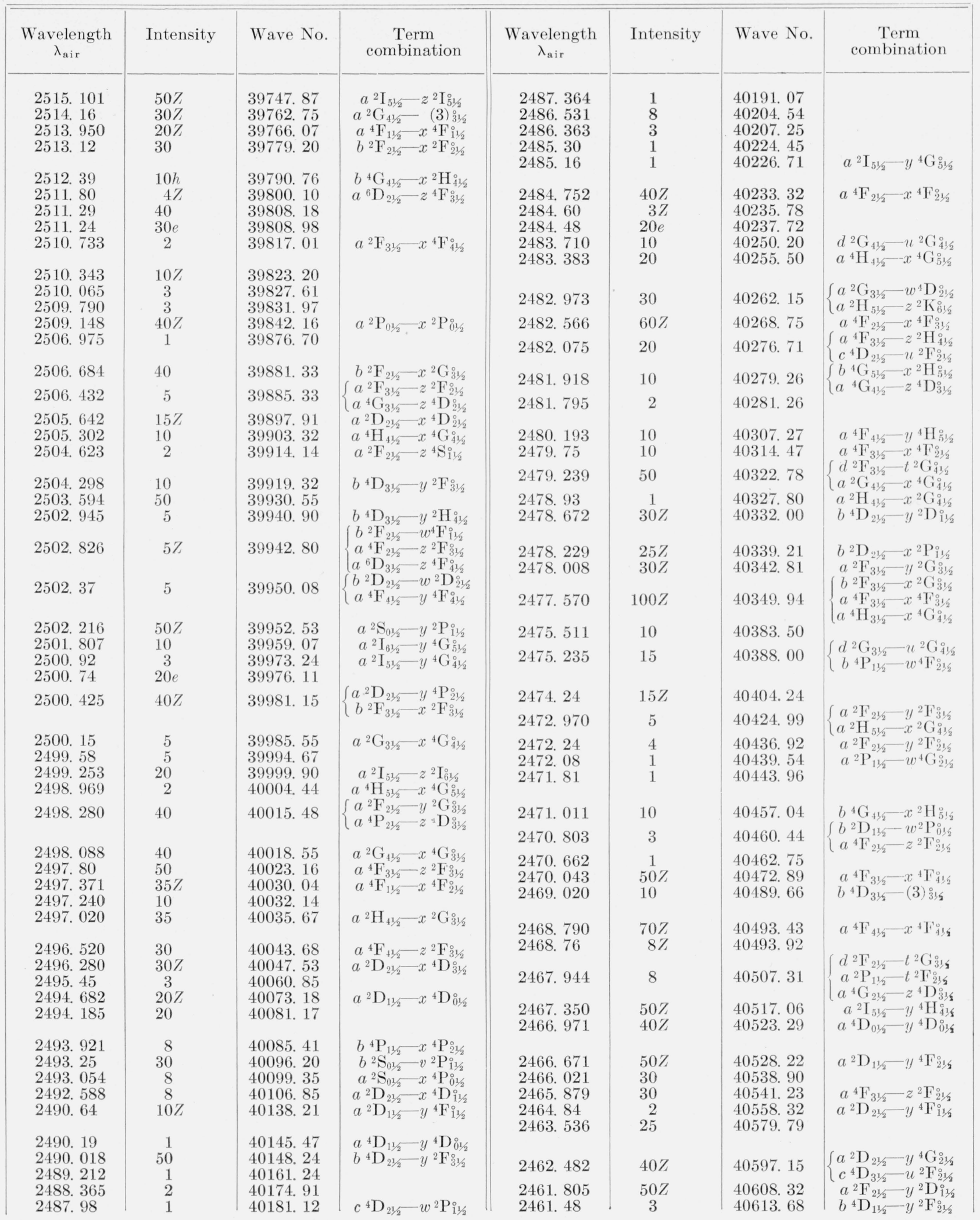


TABLE 1. Wavelengths and term combinations of Mo II-Continued

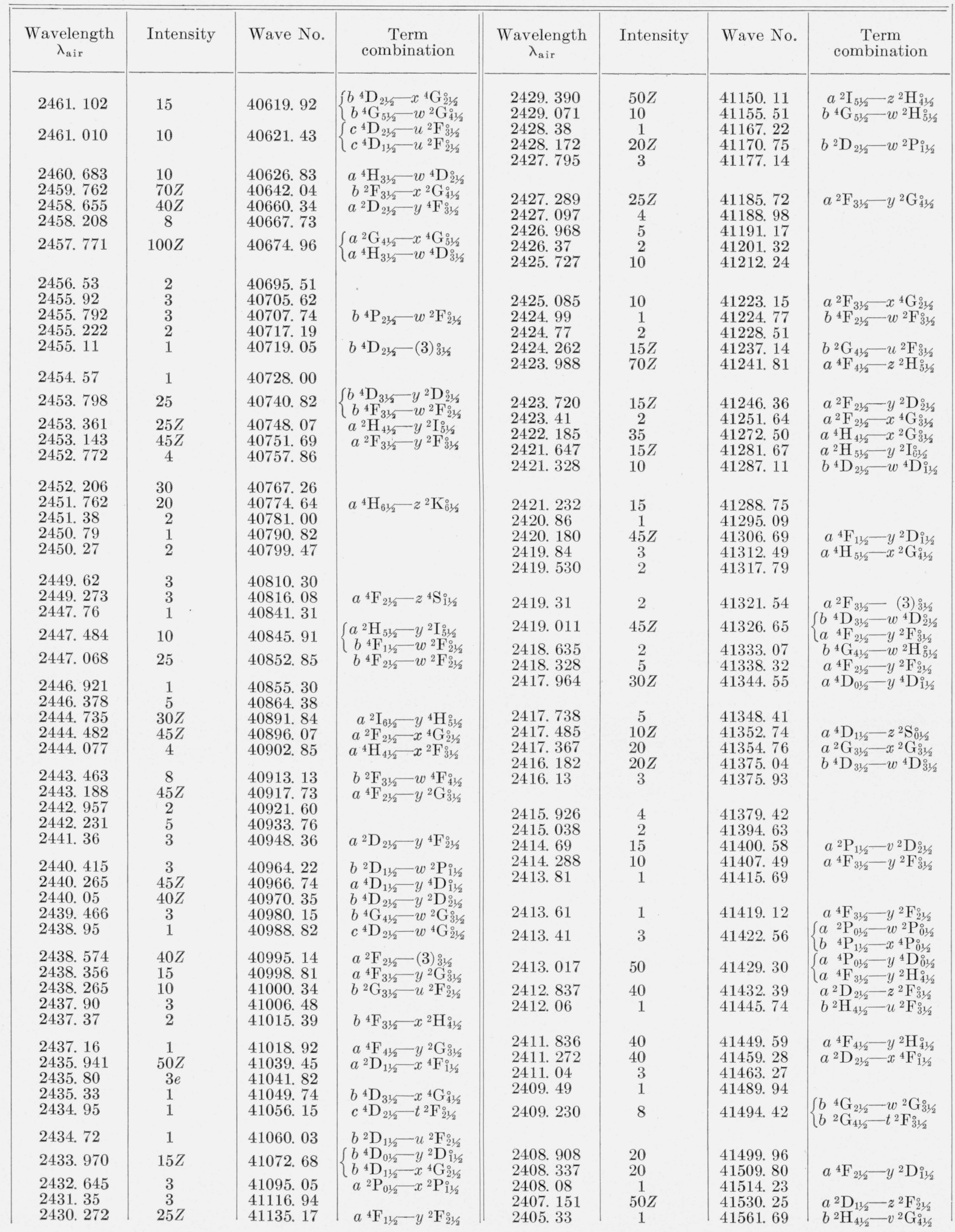


TABLE 1. Wavelengths and term combinations of Mo II-Continued

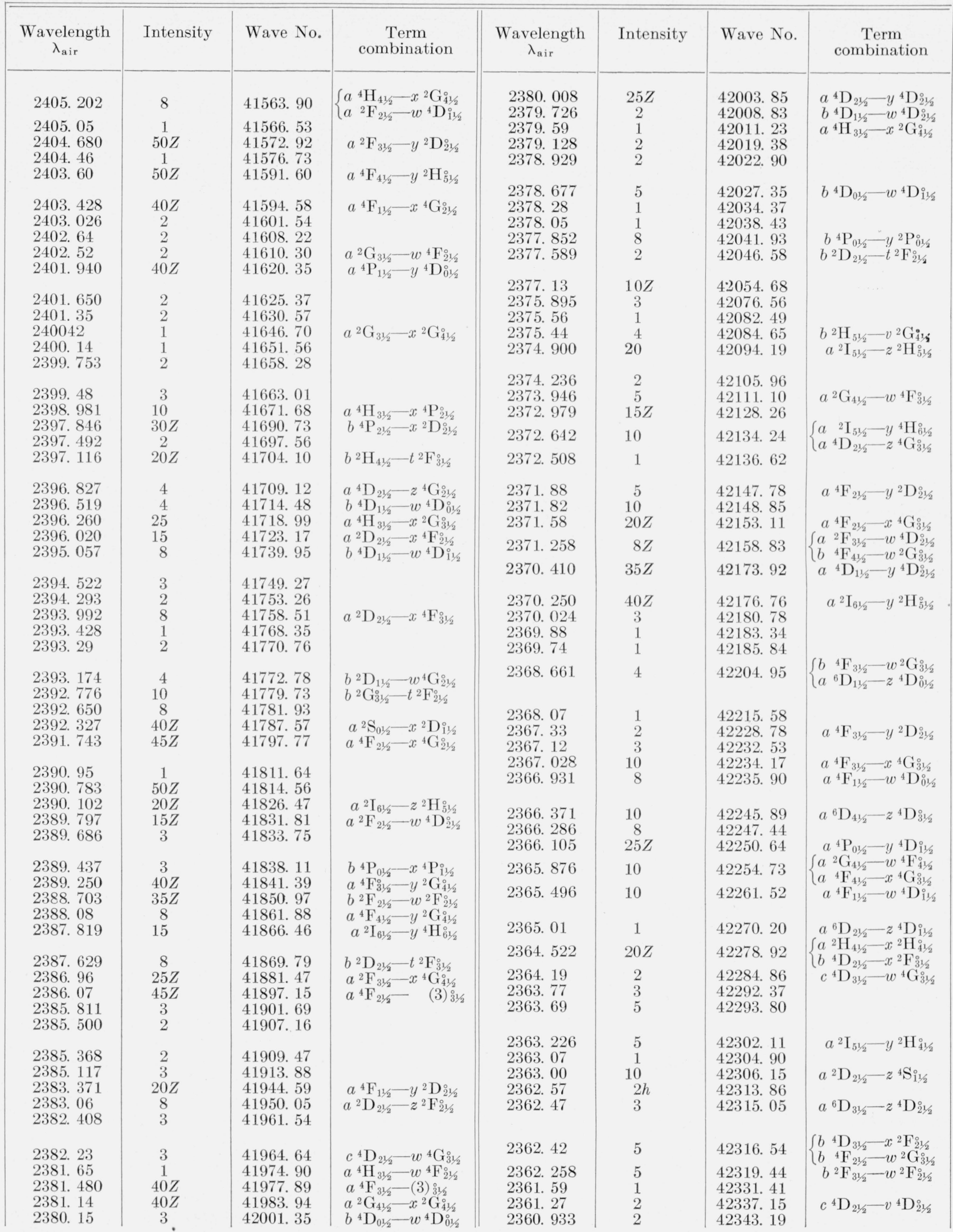


TABLE 1. Wavelengths and term combinations of Mo II-Continued

\begin{tabular}{|c|c|c|c|c|c|c|c|}
\hline $\begin{array}{c}\text { Wavelength } \\
\lambda_{\text {air }}\end{array}$ & Intensity & Wave No. & $\begin{array}{c}\text { Term } \\
\text { combination }\end{array}$ & $\begin{array}{c}\text { Wavelength } \\
\lambda_{\text {air }}\end{array}$ & Intensity & Wave No. & $\begin{array}{c}\text { Term } \\
\text { combination }\end{array}$ \\
\hline 2359. 370 & $15 Z$ & 42371. 24 & $b^{4} \mathrm{D}_{31 / 2}-x{ }^{4} \mathrm{P}_{21 / 2}^{\circ}$ & 2332.48 & 1 & 42859.68 & \\
\hline 2359. 052 & 15 & 42376.95 & $\left\{a^{2} \mathrm{H}_{41 / 2}^{01 / 2}-w^{2} \mathrm{~F}_{31 / 2}^{0}\right.$ & 2332. 307 & 3 & 42862. 85 & $a^{4} \mathrm{~F}_{31 / 2}-w^{4} \mathrm{D}_{31 / 2}^{\circ}$ \\
\hline 2358. 535 & 2 & 42386. 24 & $\left(a^{2} \mathrm{H}_{51 / 2}-x^{2} \mathrm{H}_{41 / 2}^{0}\right.$ & $\begin{array}{l}2332.131 \\
2331.192\end{array}$ & $40 Z$ & 42866. 09 & $a^{4} \mathrm{G}_{31 / 2}-z^{4} \mathrm{H}_{41 / 2}^{0}$ \\
\hline 2357.344 & $10 Z$ & $\begin{array}{l}42000.24 \\
42407.65\end{array}$ & $\left\{a^{2} \mathrm{D}_{11 / 2}-y^{2} \mathrm{~F}_{21 / 2}^{\circ}\right.$ & 2330.05 & 25 & $\begin{array}{l}42883.35 \\
42904.37\end{array}$ & $\begin{array}{l}a^{4} \mathrm{~F}_{41 / 2}-w^{4} \mathrm{D}_{31 / 2}^{01 / 2} \\
a^{6} \mathrm{D}_{01 / 2}-z^{4} \mathrm{D}_{11 / 2}^{0}\end{array}$ \\
\hline 2356. 77 & 1 & 42417.98 & $\left(a^{2} \mathrm{D}_{21 / 2}-y^{2} \mathrm{G}_{31 / 2}^{\circ}\right.$ & 2330.03 & 5 & 42904. 74 & \\
\hline & & & & 2329. 708 & $50 Z$ & 42910.67 & $a^{4} \mathrm{~F}_{41 / 2}-x{ }^{4} \mathrm{G}_{51 / 2}^{\circ}$ \\
\hline 2356. 46 & 1 & 42423.56 & & 2328.95 & 8 & 42924. 63 & $a^{2} \mathrm{~F}_{21 / 2}-x^{2} \mathrm{G}_{31 / 2}^{0}$ \\
\hline 2356. 06 & 5 & 42430.76 & $b^{2} \mathrm{G}_{41 / 2}-v^{2} \mathrm{G}_{41 / 2}^{\circ}$ & 2328.89 & 1 & 42925. 74 & \\
\hline $\begin{array}{l}2355.460 \\
2355.309\end{array}$ & $\begin{array}{l}30 Z \\
15\end{array}$ & $\begin{array}{l}42441.57 \\
42444.29\end{array}$ & $\begin{array}{c}a^{4} \mathrm{P}_{11 / 2}-y^{4} \mathrm{D}_{11 / 2}^{11} \\
a^{2} \mathrm{I}_{11}-y^{2} \mathrm{H}^{\circ}\end{array}$ & 2328.367 & 2 & 42935. 38 & \\
\hline 2355.022 & 15 & 42449. 46 & $a^{4} \mathrm{P}_{21 / 2}-y^{4} \mathrm{D}_{11 / 2}^{11 / 2}$ & $\begin{array}{l}2328.115 \\
2327.815\end{array}$ & $\begin{array}{l}8 Z \\
4\end{array}$ & $\begin{array}{l}42940.03 \\
42945.56\end{array}$ & $\begin{array}{l}b^{2} \mathrm{D}_{21 / 2}-v^{2} \mathrm{D}_{21 / 2}^{\circ} \\
a^{2} \mathrm{H}_{41}-x^{2} \mathrm{H}^{\circ}\end{array}$ \\
\hline 2354. 702 & 15 & 42455. 23 & $a^{6} \mathrm{D}_{01 / 2}-z^{4} \mathrm{D}_{01 / 2}^{\circ}$ & 2326. 93 & 1 & 42961.89 & \\
\hline 2354. 186 & 20 & 42464.53 & $\left\{a^{4} \mathrm{G}_{41 / 2}-z^{4} \mathrm{H}_{31 / 2}^{0}\right.$ & 2325. 86 & 8 & 42981. 65 & $b^{4} \mathrm{D}_{31 / 2}-w^{4} \mathrm{~F}_{41 / 2}^{\circ}$ \\
\hline 2351.547 & 1 & 42512,18 & 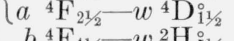 & 2324.876 & 50 & 42999.85 & $a^{2} \mathbf{D}_{21 / 2}-y^{2} \mathbf{D}_{11 / 2}^{0}$ \\
\hline 2351. 132 & 1 & $\begin{array}{l}42012.18 \\
42519.69\end{array}$ & $b^{4} \mathrm{~F}_{41 / 2}-w^{2} \mathrm{H}_{51 / 2}^{1 / 2}$ & 2324. 29 & 1 & 43010. 69 & \\
\hline 2350.550 & 5 & 42530. 22 & $a^{4} \mathrm{~F}_{11 / 2}-w^{4} \mathrm{D}_{21 / 2}^{0}$ & $\begin{array}{l}2323.96 \\
2322.971\end{array}$ & $\begin{array}{l}50 \\
10\end{array}$ & $\begin{array}{l}\text { 43016. } 79 \\
\text { 43035. } 10\end{array}$ & $\begin{array}{l}b{ }^{4} \mathrm{P}_{21 / 2}-w^{2} \mathrm{D}_{11 / 2}^{\circ}{ }^{i} \mathrm{D}_{31 / 2}-w^{4} \mathrm{G}_{411}^{\circ}\end{array}$ \\
\hline 2350.120 & $25 Z$ & 42538.00 & $a^{4} \mathrm{~F}_{31 /}-x^{4} \mathrm{G}_{41 / 6}$ & 2322. 499 & $25 Z$ & 43043.85 & $a^{2} \mathrm{H}_{51 / 2}-x^{2} \mathrm{H}_{51 / 2}^{\circ}$ \\
\hline 2349. 005 & 10 & 42558. 19 & $a^{4} \mathrm{~F}_{41 / 2}^{3 / 2}-x^{4} \mathrm{G}_{41 / 2}^{0}$ & 2321. 955 & 1 & 43053. 93 & $b{ }^{4} \mathrm{D}_{11 / 2}-x^{4} \mathrm{P}_{21 / 2}^{\circ}$ \\
\hline 2348.832 & $20 Z$ & 42561.32 & $a^{4} \mathrm{G}_{31 / 2}^{11 / 2}-z^{4} \mathrm{H}_{31 / 2}^{41 / 2}$ & 2321.67 & 2 & 43059. 22 & \\
\hline 2347. 802 & $30 Z$ & 42579. 99 & $\begin{cases}a^{2} \mathrm{D}_{11 / 2}-y^{2} \mathrm{D}_{11 / 2}^{\circ} \\
b^{2} \mathrm{G}_{41}-w^{4} \mathrm{G}^{\circ}\end{cases}$ & 2321. 23 & 2 & 43067. 38 & $b^{4} \mathrm{D}_{21 / 2}-w^{4} \mathbf{F}_{31 / 2}^{\circ}$ \\
\hline 2347.07 & 5 & 42593. 27 & $b^{2} \mathrm{~F}_{31 / 2}-x^{2} \mathrm{H}_{41 / 2}^{21 / 2}$ & 2321. 06 & 3 & 43070.53 & $\left(a^{2} \mathrm{~S}_{01}-w^{2} \mathrm{D}^{\circ}\right.$ \\
\hline 2346.673 & 10 & 42600.48 & & 2320. 090 & 30 & 43088. 54 & $\left\{\begin{array}{l}c^{4} \mathrm{D}_{31 / 2}-v^{4} \mathrm{~F}_{41 / 2}^{11 / 2}\end{array}\right.$ \\
\hline 2345.80 & $1 \mathrm{w}$ & $\begin{array}{l}42600.48 \\
42616.33\end{array}$ & $b^{4} \mathrm{D}_{21 / 2}-x^{4} \mathrm{P}_{21 / 2}^{\circ}$ & 2318. 897 & 2 & 43110. 71 & $b^{4} \mathrm{P}_{11 / 2}-x^{2} \mathrm{D}_{11 / 2}^{0}$ \\
\hline $\begin{array}{l}2345.699 \\
2345.54\end{array}$ & 2 & 42618. 16 & & 2316. 477 & $25 Z$ & 43155. 74 & \\
\hline $\begin{array}{l}2345.54 \\
2345.27\end{array}$ & $\begin{array}{l}1 \\
1\end{array}$ & & & 2316.040 & 10 & 43163.88 & \\
\hline & & & & $\begin{array}{l}2315.632 \\
2315.087\end{array}$ & $\begin{array}{c}25 Z \\
5\end{array}$ & $\begin{array}{l}43171.48 \\
43181.65\end{array}$ & $a^{6} \mathrm{D}_{11 / 2}-z^{4} \mathrm{D}_{21 / 6}$ \\
\hline $\begin{array}{l}2344.676 \\
2344.33\end{array}$ & $\begin{array}{c}10 Z \\
3\end{array}$ & $\begin{array}{l}42636.76 \\
42643.05\end{array}$ & $a{ }^{4} \mathrm{P}_{01 / 2}-z{ }^{2} \mathrm{~S}_{01 / 2}^{\circ}$ & 2313.878 & 15 & 43204. 21 & $a^{2} \mathrm{~F}_{31 / 2}^{11 / 2}-x^{4} \mathrm{P}_{21 / 2}^{21 / 2}$ \\
\hline 2343. 880 & 1 & 42651. 23 & & 2313. 154 & 10 & 43217. 73 & $a^{2} \mathrm{D}_{11 / 2}-y{ }^{2} \mathrm{D}_{21 / 2}^{\circ}$ \\
\hline 2343. 752 & 10 & 42653.56 & $a^{6} \mathrm{D}_{11 / 2}-z^{4} \mathrm{D}_{11 / 2}^{\circ}$ & 2312.927 & 2 & 43221. 97 & \\
\hline 2343.54 & 3 & 42657.42 & $c^{4} \mathrm{D}_{31 / 2}^{11 / 2}-v^{4} \mathrm{D}_{21 / 2}^{11 / 2}$ & $\begin{array}{l}2312.233 \\
2311.382\end{array}$ & $\begin{array}{l}10 \\
20\end{array}$ & $\begin{array}{l}43234.94 \\
43250.86\end{array}$ & $a^{2} \mathrm{~F}_{31}-x^{2} \mathrm{G}_{31}$ \\
\hline 2343. 04 & 4 & 42666.50 & $a^{4} \mathrm{D}_{11 / 2}-z^{2} \mathrm{D}_{11 / 2}^{\circ}$ & 2310.85 & 1 & 43260.82 & $x^{-1} x_{31 / 2} \quad x-431 / 2$ \\
\hline 2342. 616 & 10 & 42674. 25 & $b^{4} \mathrm{D}_{31 / 2}-w^{4} \mathrm{~F}_{21 / 2}$ & & & & \\
\hline $\begin{array}{l}\text { 2342. } 292 \\
2341.57\end{array}$ & $\begin{array}{c}8 \\
40 Z\end{array}$ & $\begin{array}{l}42680.15 \\
42693.31\end{array}$ & $4{ }^{4} \mathrm{G}=4 \mathrm{H}^{\circ}$ & $\begin{array}{l}2310.00 \\
2309.485\end{array}$ & 20 & $\begin{array}{l}43264.37 \\
43286.38\end{array}$ & $\begin{array}{l}a{ }^{4} \mathrm{H}_{51 / 2}-x^{2} \mathrm{H}_{41 / 2}^{0} \\
a^{2} \mathrm{H}^{4}-w^{2} \mathrm{G}^{\circ}\end{array}$ \\
\hline 2341. 20 & $\begin{array}{c}40 Z \\
1\end{array}$ & 42700.05 & $a^{4} \mathrm{G}_{21 / 2}-z^{7} \mathrm{H}_{31 / 2}^{1 / 2}$ & $\begin{array}{l}2309.40 \\
2309.33\end{array}$ & 1 & $\begin{array}{l}43287.95 \\
43289.26\end{array}$ & $a^{2} \mathrm{D}_{21 / 2}-x^{4} \mathrm{G}_{21 / 2}$ \\
\hline 2340.88 & 1 & 42705.89 & & $\begin{array}{l}2309.35 \\
2308.897\end{array}$ & $\begin{array}{l}1 \\
2\end{array}$ & $\begin{array}{l}43289.20 \\
43297.21\end{array}$ & $a^{0} D_{21 / 2}-z+D_{31 / 2}^{1 / 2}$ \\
\hline 2340.41 & $35 Z$ & 42714.47 & $a^{2} \mathrm{I}_{51 / 2}-y^{2} \mathrm{G}_{41 / 2}^{\circ}$ & 2308. 114 & 1 & 43312.07 & \\
\hline 2339.376 & 8 & 42733. 34 & $\begin{cases}a^{4} \mathrm{D}_{31 / 2}-z^{4} \mathrm{I}_{41 / 2} \\
a & 4 \mathrm{~F}^{4}-w^{4} \mathrm{D}^{4}\end{cases}$ & 2307. 994 & $30 Z$ & 43314. 31 & 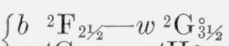 \\
\hline 2339. 190 & 5 & 42736. 74 & 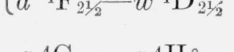 & 2307.178 & 1 & 43329. 63 & $\begin{aligned}\left(a^{a}{ }^{4} \mathrm{G}_{51 / 2}\right. & z^{4} \mathrm{H}_{51 / 2}^{0} b^{4} \mathrm{D}^{4}-x^{4} \mathrm{P}_{0}^{0}\end{aligned}$ \\
\hline 2338. 482 & 10 & 42749.68 & $a^{4} \mathrm{G}_{51 / 2}-z^{4} \mathrm{H}_{41 / 2}^{\circ}$ & 2306. 990 & $50 Z$ & 43333. 16 & $a^{4} \mathrm{G}_{41 / 2}^{2 / 2}-z{ }^{4} \mathrm{H}_{51 / 2}^{1 / 2}$ \\
\hline 2337.67 & 3 & 42764.53 & & 2305.874 & 8 & 43354. 14 & $a{ }^{4} \mathrm{P}_{11 / 2}-z{ }^{4} \mathrm{G}_{21 / 2}^{\circ}$ \\
\hline 2337. 432 & $15 Z$ & 42768.88 & $a^{4} \mathrm{G}_{41 / 2}-z^{4} \mathrm{H}_{41 / 2}^{\circ}$ & 2305. 673 & $30 Z$ & 43357. 92 & $a^{4} \mathrm{D}_{21 / 2}-y{ }^{4} \mathrm{D}_{31 / 2}^{\circ}$ \\
\hline $\begin{array}{l}2337.053 \\
2336.68\end{array}$ & $\begin{array}{l}5 \\
1\end{array}$ & $\begin{array}{l}42775.82 \\
42782,65\end{array}$ & $c^{4} \mathrm{D}_{31 / 2}-v^{4} \mathrm{D}_{31 / 2}^{0}$ & 2305. 453 & $\begin{array}{l}5 \\
8\end{array}$ & 43362. 05 & $a^{4} \mathrm{P}_{21 / 2}^{272}-z^{4} \mathrm{G}_{21 / 2}^{01 / 2}$ \\
\hline 2336. 220 & 10 & $\begin{array}{l}42782.05 \\
42791.07\end{array}$ & $a^{4} \mathrm{D}_{21 / 2}-z^{2} \mathrm{G}_{31 / 2}^{1 / 2}$ & $\begin{array}{l}2305.276 \\
2304.261 \\
2302.995\end{array}$ & $\begin{array}{c}8 \\
80 Z \\
8\end{array}$ & $\begin{array}{l}43365.38 \\
43384.48 \\
43408.33\end{array}$ & $a^{2} \mathrm{H}_{51 / 2}-w^{2} \mathrm{G}_{41 / 2}^{\circ}$ \\
\hline 2335. 81 & 10 & 42798.58 & $a^{6} \mathrm{D}_{21 / 2}-z^{4} \mathrm{D}_{21 / 2}^{\circ}$ & & & & \\
\hline 2335. 392 & 10 & 42806. 24 & $a^{6} \mathrm{D}_{31 / 2}^{2 / 2}-z \times \mathrm{D}_{31 / 2}^{0,2}$ & $\begin{array}{l}2302.848 \\
2302.48\end{array}$ & $\begin{aligned} 20 \\
2\end{aligned}$ & 43411. 13 & $a^{2} \mathrm{I}_{51 / 2}-x^{4} \mathrm{G}_{41 / 2}^{\circ}$ \\
\hline 2335. 18 & 1 & 42810. 12 & $b^{4} \mathrm{G}_{31 / 2}-w^{2} \mathrm{D}_{21 / 2}^{0}$ & 2302143 & $\begin{array}{l}2 \\
8\end{array}$ & $\begin{array}{l}43418.06 \\
43424 \quad 42\end{array}$ & \\
\hline 2334. 942 & $20 Z$ & 42814.49 & $a^{4} \mathrm{~F}_{31 / 2}-w^{4} \mathrm{D}_{21 / 2}$ & 2301.004 & $\begin{array}{l}0 \\
2\end{array}$ & $\begin{array}{l}43424.42 \\
43445.91\end{array}$ & \\
\hline 2334.84 & $20 Z$ & 42816.36 & $a^{2} \mathrm{D}_{21 / 2}-y^{2} \mathrm{~F}_{31 / 2}^{\circ}$ & 2300.697 & 1 & 43451. 71 & \\
\hline 2334. 22 & 3 & 42827.73 & $a{ }^{4} \mathrm{P}_{11 / 2}-z^{2} \mathrm{~S}_{01 / 2}^{0}$ & 2300.41 & 1 & 43457. 13 & $a^{4} \mathrm{~F}_{21 / 2}-x{ }^{2} \mathrm{~F}_{31 / 2}^{\circ}$ \\
\hline 2334. 066 & 5 & 42830.55 & & 2300. 096 & 2 & 43463. 06 & \\
\hline 2333. 870 & 3 & 42834. 15 & $b^{2} \mathrm{~F}_{21 / 2}-x^{2} \mathrm{D}_{21 / 2}^{\circ}$ & 2299. 876 & 10 & 43467. 22 & \\
\hline $\begin{array}{l}2333.650 \\
2332.67\end{array}$ & $\begin{array}{l}5 \\
3\end{array}$ & $\begin{array}{l}42838.19 \\
42856.18\end{array}$ & $b^{4} \mathrm{D}_{31 / 2}-w^{4} \mathrm{~F}_{31 / 2}^{1}$ & $\begin{array}{l}2299.657 \\
2298.888\end{array}$ & $\begin{array}{l}1 \\
3\end{array}$ & $\begin{array}{l}43471.36 \\
43485.90\end{array}$ & \\
\hline
\end{tabular}


TABLE 1. Wavelengths and term combinations of Mo II-Continued

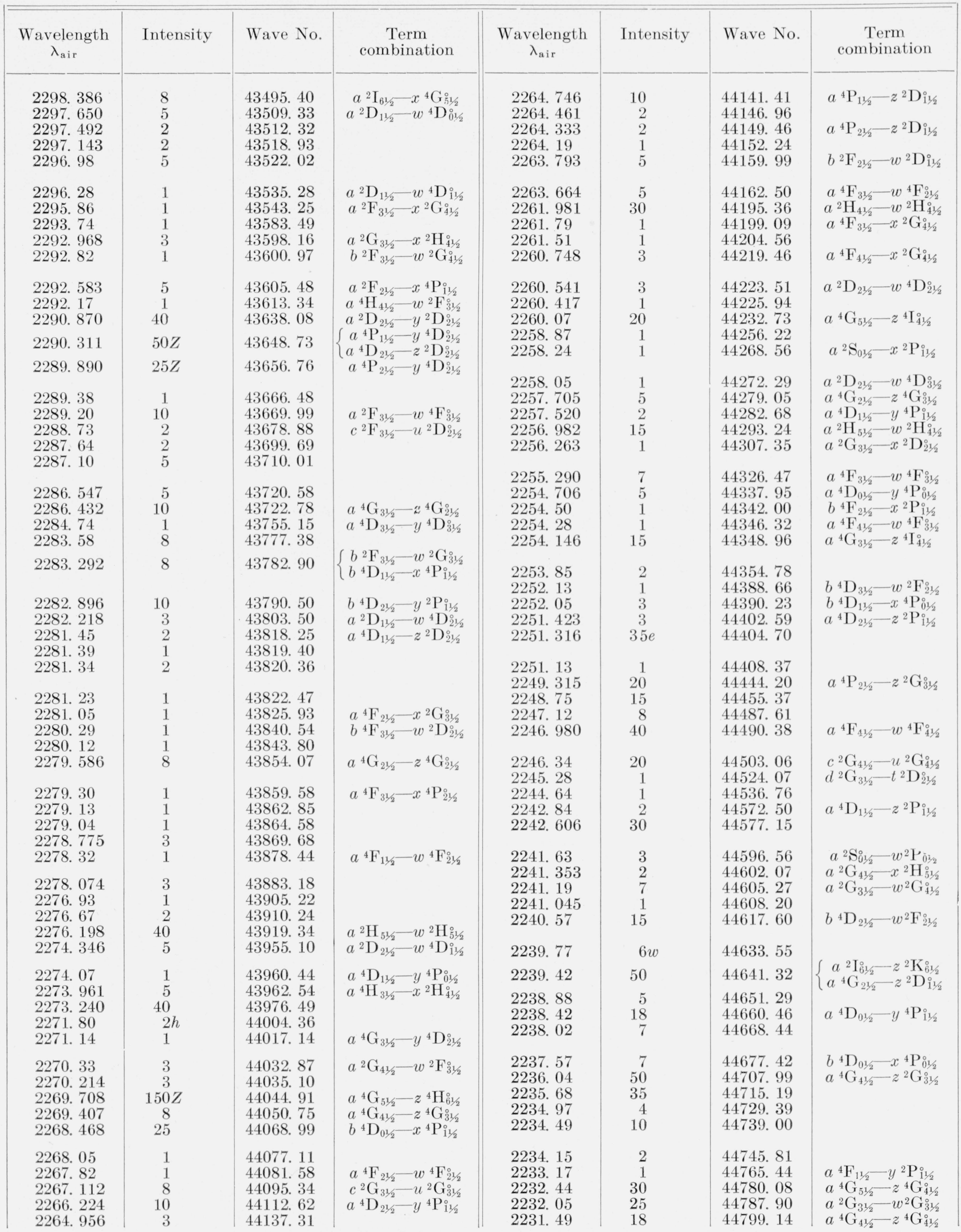


TABLE 1. Wavelengths and term combinations of Mo II-Continued

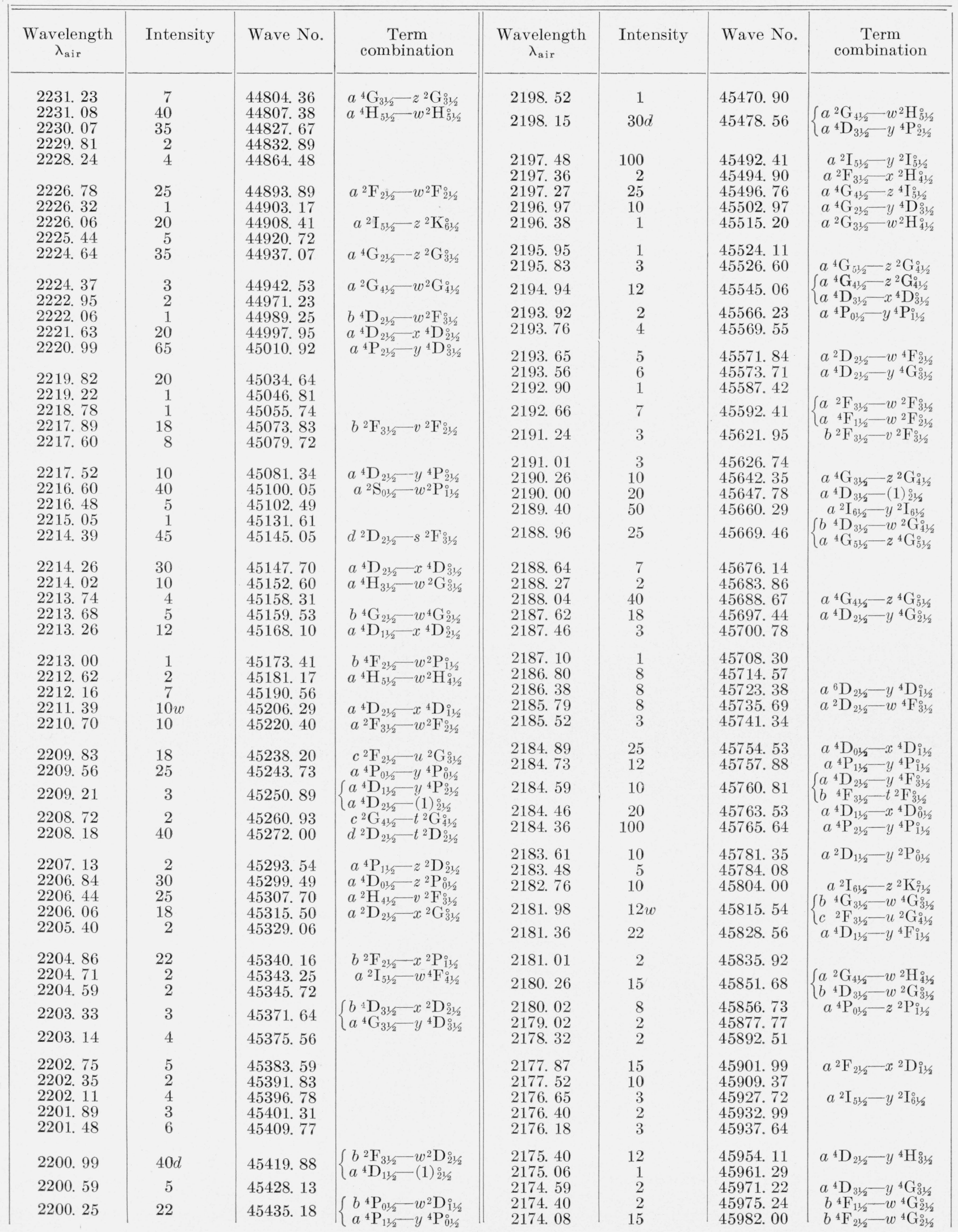


TABLE 1. Wavelengths and term combinations of Mo II-Continued

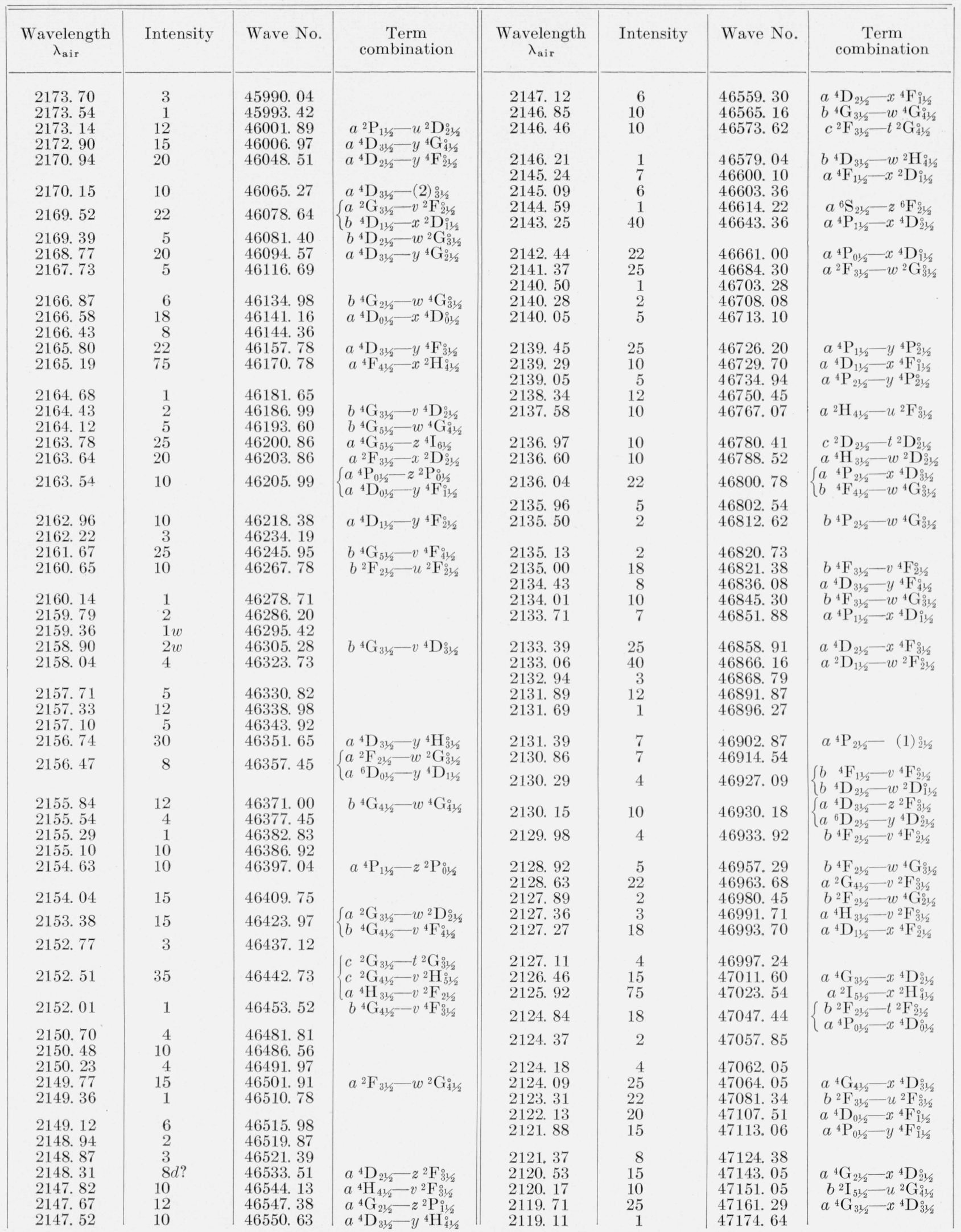


TABLE 1. Wavelengths and term combinations of Mo II-Continued

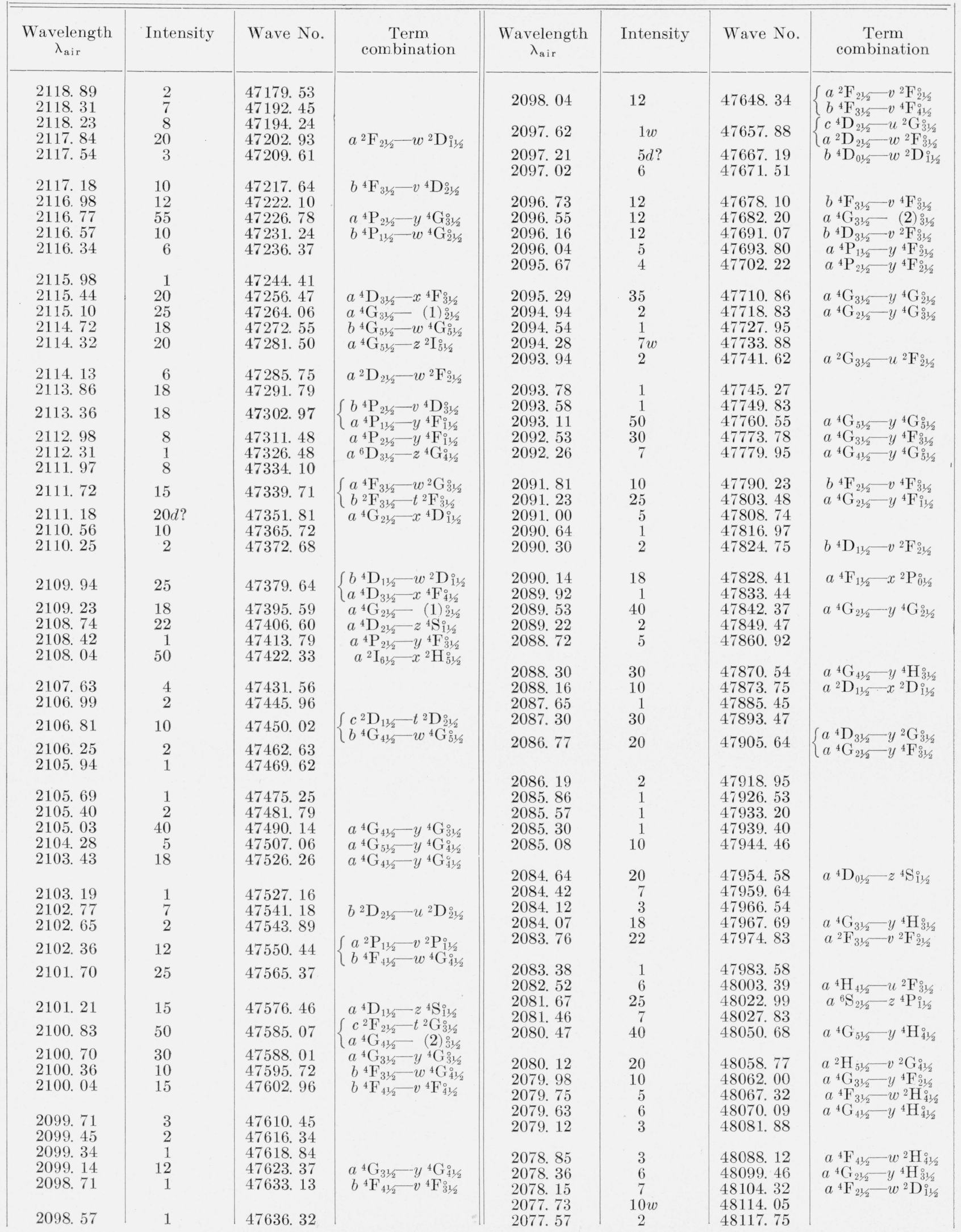


TABLE 1. Wavelengths and term combinations of Mo II-Continued

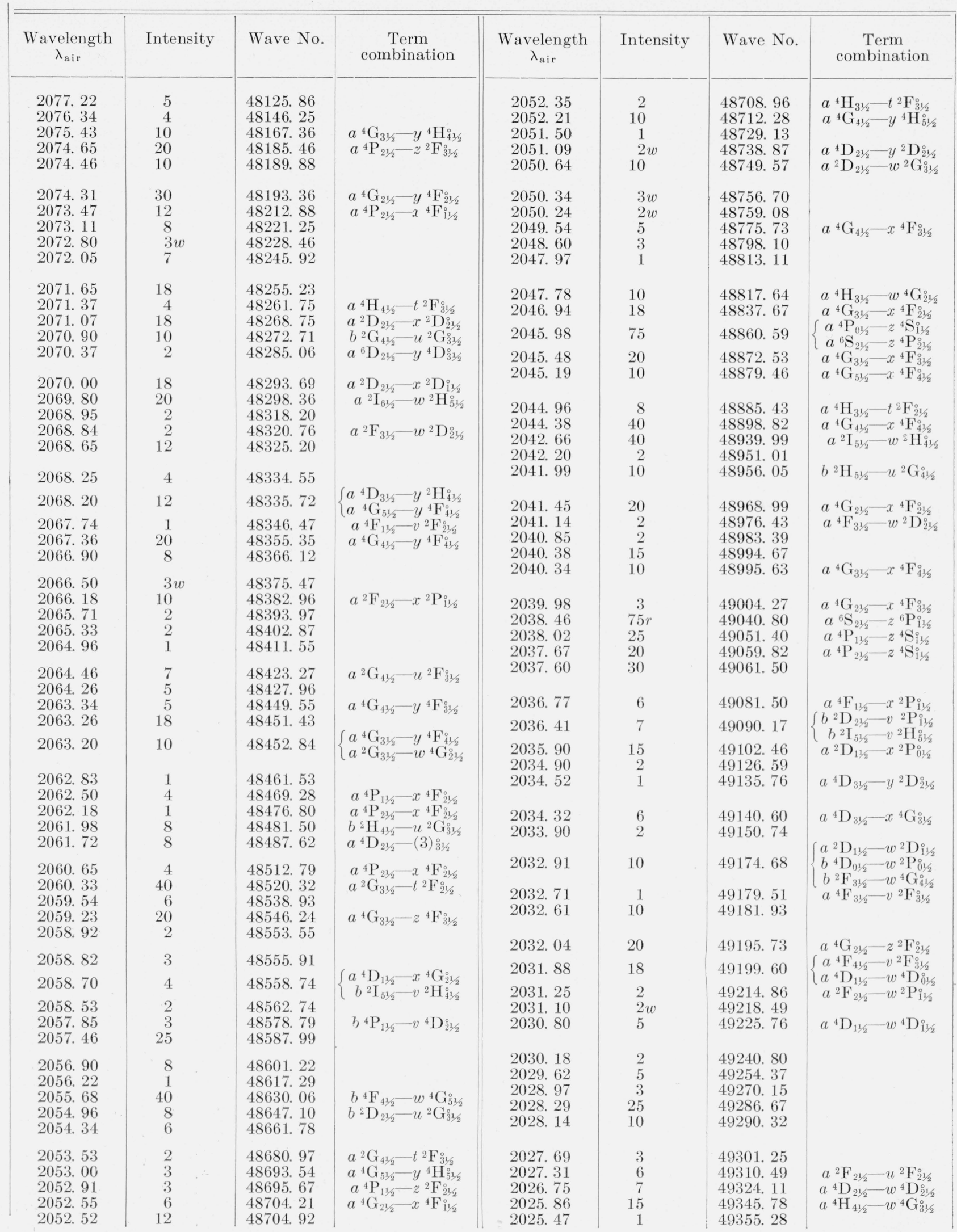


TABLE 1. Wavelengths and term combinations of Mo II-Continued

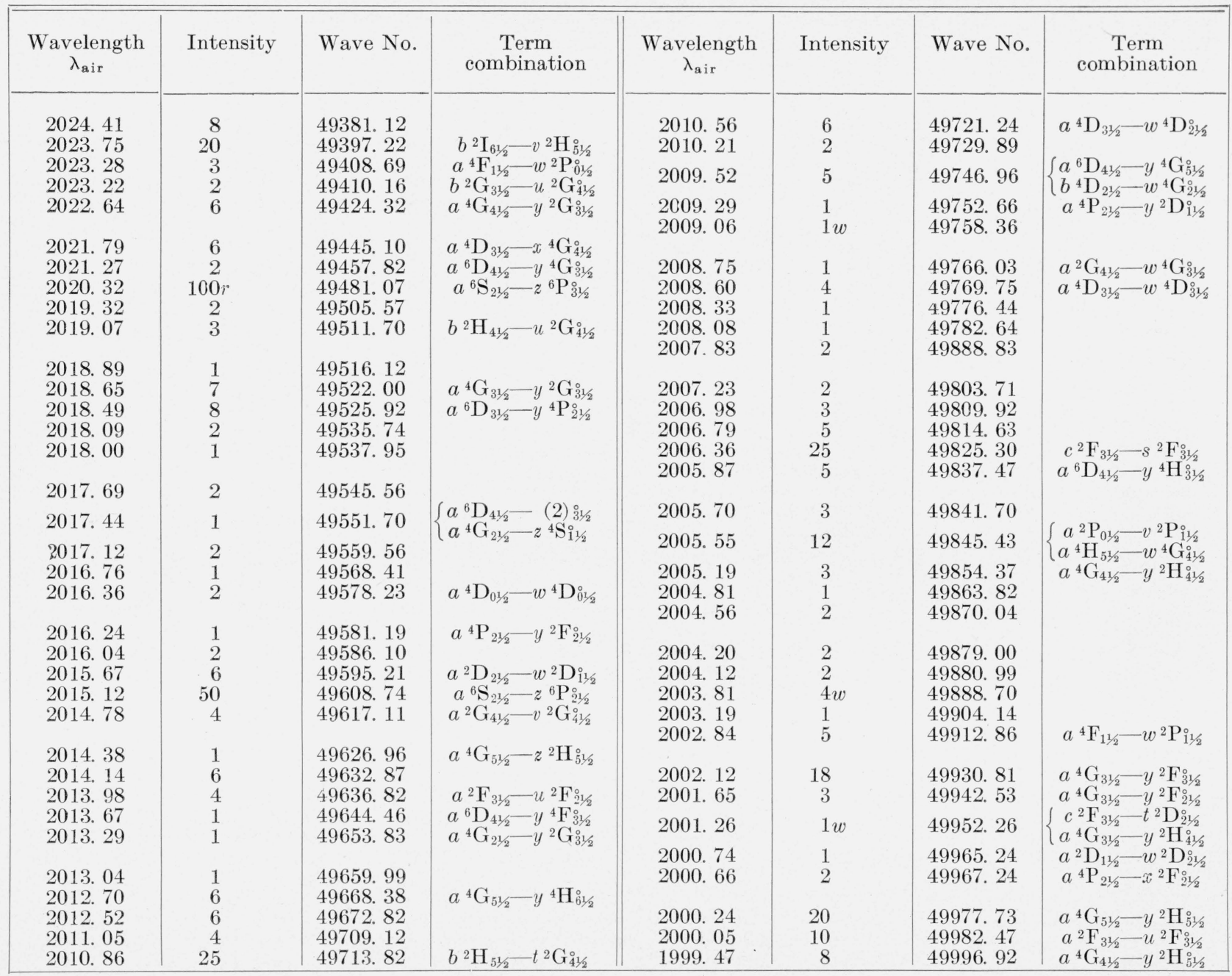

TABLE 2. Wavelengths of Mo II in vacuum

\begin{tabular}{|c|c|c|c|c|c|c|c|}
\hline $\begin{array}{l}\text { Wavelength } \\
\text { computed }\end{array}$ & Intensity & Wave No. & $\begin{array}{c}\text { Term combina- } \\
\text { tion }\end{array}$ & $\begin{array}{l}\text { Wavelength } \\
\text { computed }\end{array}$ & Intensity & Wave No. & $\begin{array}{c}\text { Term combina- } \\
\text { tion }\end{array}$ \\
\hline 1998. 41 & 12 & 50039.83 & $a^{2} \mathrm{D}_{21 / 2}-v^{2} \mathrm{~F}_{21 / 2}^{0}$ & 1985. 94 & 4 & 50354.61 & $a^{2} \mathrm{D}_{11 / 2}-x^{2} \mathrm{P}_{11 / 2}^{\circ}$ \\
\hline 1997.54 & 10 & 50061.70 & $\left\{\begin{array}{l}a^{4} \mathrm{G}_{21 / 2} y^{2} \mathrm{~F}_{31 / 2}^{\circ} \\
a^{6} \mathrm{D}_{11 / 2}-z^{2} \mathrm{P}_{01 / 2}^{31}\end{array}\right.$ & 1985.53 & 5 & 50364.35 & $\left\{\begin{array}{l}b{ }^{2} \mathrm{H}_{51 / 2}-v^{2} \mathrm{H}_{41 / 2}^{0} \\
a^{4} \mathrm{G}_{31 / 2}-y^{2} \mathrm{G}_{41 / 1}^{0}\end{array}\right.$ \\
\hline 1997. 02 & 5 & 50074.50 & $\left\{\begin{array}{l}a^{4} \mathrm{G}_{21 / 2}-y^{2} \mathrm{~F}_{21 / 2}^{\circ} \\
a^{6} \mathrm{D}_{21 / 2}-x^{4} \mathrm{D}_{31 / 2}^{\circ}\end{array}\right.$ & $\begin{array}{l}1985.35 \\
1984.70\end{array}$ & $\begin{array}{r}4 \\
15\end{array}$ & $\begin{array}{l}50368.82 \\
50385.42\end{array}$ & $\begin{array}{l}a^{4} \mathrm{D}_{21 / 2}-x^{4} \mathrm{P}_{21 / 2}^{2} \\
a^{2} \mathrm{D}_{21 / 2}-w^{2} \mathrm{D}_{21 / 2}^{2}\end{array}$ \\
\hline $\begin{array}{l}1996.40 \\
1995.53\end{array}$ & $\begin{array}{r}18 \\
1\end{array}$ & $\begin{array}{l}50090.08 \\
50111.91\end{array}$ & $\begin{array}{l}a^{2} \mathrm{~F}_{21 / 2}-t^{2} \mathrm{~F}_{21 / 2}^{012} \\
a^{6} \mathrm{D}_{31 / 2}-(2)_{31 / 2}^{2}\end{array}$ & 1984. 47 . & 2 & 50391. 25 & $\begin{cases}a & { }^{4} \mathrm{P}_{21 / 2}-y^{2} \mathrm{D}_{21 / 2}^{2} \\
a^{6}{ }^{6} \mathrm{D}_{11 / 2}-y & { }^{4} \mathrm{P}_{21 / 2}^{2}\end{cases}$ \\
\hline $\begin{array}{l}1994.66 \\
1994.42\end{array}$ & $\begin{array}{r}15 \\
5\end{array}$ & $\begin{array}{l}50133.76 \\
50139.87\end{array}$ & $\begin{array}{l}a^{6} \mathrm{D}_{21 / 2}-x^{4} \mathrm{D}_{11 / 2}^{\circ} \\
a^{4} \mathrm{P}_{21 / 2}-(3)_{31 / 2}^{11}\end{array}$ & $\begin{array}{l}1984.06 \\
1983.48\end{array}$ & 10 & $\begin{array}{l}50401.67 \\
50416.41\end{array}$ & $\begin{array}{l}a^{4} \mathrm{G}_{31 / 2}-x^{4} \mathrm{G}_{21 / 2}^{0} \\
\left\{\begin{array}{l}a^{4} \mathrm{D}_{21 / 2}-x^{2} \mathrm{G}_{31 / 2}^{0} \\
a^{6} \mathrm{D}_{41 / 2}-z^{2} \mathrm{~F}_{31 / 2}^{0}\end{array}\right.\end{array}$ \\
\hline 1992. 89 & 3 & 50178.38 & $\left\{\begin{array}{l}a^{6}{ }^{6} \mathrm{D}_{21 / 2}-(1)_{21 / 2}^{\circ} \\
a^{2} \mathrm{G}_{31 / 2}-w^{4} \mathrm{G}_{41 / 2}^{2}\end{array}\right.$ & 1981.08 & 1 & 50477.50 & $\mid \begin{array}{l}a^{a}{ }^{2} \mathrm{~F}_{31 / 2}-t^{2} \mathrm{~F}_{21 / 2}^{0} \\
a^{4} \mathrm{D}_{21 / 2}-w^{4} \mathrm{~F}_{11 / 2}^{1}\end{array}$ \\
\hline 1992. 35 & 10 & 50192. 00 & $a^{6} \mathrm{~S}_{21 / 2}-z^{6} \mathrm{D}_{11 / 2}^{112}$ & 1980. 49 & 7 & 50492.49 & $\left\{\begin{array}{l}b^{4} \mathrm{D}_{31 / 2}-w^{4} \mathrm{G}_{31 / 2} \\
0\end{array}\right.$ \\
\hline 1992. 07 & 2 & 50199.16 & $b^{4} \mathrm{D}_{11 / 2}-w^{4} \mathrm{G}_{21 / 2}^{8}$ & 1979. 83 & 2 & 50509.32 & $\left(\begin{array}{c}a a^{0} \mathrm{D}_{31 / 2}-y^{4} \mathrm{~F}_{21 / 2}^{21 / 2} \\
a^{4} \mathrm{P}_{01 / 2}-w^{4} \mathrm{D}_{11 / 2}^{0}\end{array}\right.$ \\
\hline $\begin{array}{l}\text { 1990. } 44 \\
1990.25\end{array}$ & $\begin{array}{r}15 \\
8\end{array}$ & $\begin{array}{l}50240.25 \\
50245.05\end{array}$ & $\begin{array}{l}a^{2} \mathrm{~F}_{31 / 2}-t^{2} \mathrm{~F}_{31 / 2}^{\circ} \\
a^{4} \mathrm{G}_{21 / 2}-y^{2} \mathrm{D}_{11 / 2}^{\circ}\end{array}$ & 1979.51 & 1 & 50516.49 & $\begin{cases}a & { }^{c} \mathrm{G}_{41 / 2}-w^{4} \mathrm{C}_{41 / 2}^{\circ} \\
a & 6 \mathrm{D}_{11-{ }^{4}} \mathrm{D}^{\circ 11}\end{cases}$ \\
\hline 1990.13 & 15 & 50247.94 & $a^{4} \mathrm{G}_{51 / 2}-y^{2} \mathrm{G}_{41 / 2}^{0}$ & 1978. 91 & 15 & 50532.98 & $a^{4} \mathrm{G}_{21 / 2}^{1 / 2}-x^{4} \mathrm{G}_{21 / 2}^{11 / 2}$ \\
\hline 1989. 37 & 12 & 50267.07 & $\left\{\begin{array}{l}b{ }^{4} \mathrm{D}_{11 / 2}-t^{2} \mathrm{~F}_{21 / 2}^{\circ} \\
a^{4} \mathrm{G}_{41 / 2}-y^{2} \mathrm{G}_{41 / 2}^{2}\end{array}\right.$ & $\begin{array}{l}1978.68 \\
1978.24\end{array}$ & $\begin{array}{r}2 \\
18\end{array}$ & $\begin{array}{l}50538.82 \\
50550.10\end{array}$ & $\begin{array}{l}a^{4} \mathrm{D}_{11 / 2}-x^{4} \mathrm{P}_{21 / 2}^{0} \\
a^{4} \mathrm{H}^{2}-w^{4} \mathrm{G}^{0}\end{array}$ \\
\hline 1987. 97 & 20 & 50302.54 & $a^{6} \mathrm{~S}_{21 / 2}-z^{6} \mathrm{D}_{31 / 2}^{1}$ & 1977. 17 & 20 & 50577. 34 & $a^{6} \mathrm{~S}_{21 / 2}-z^{6} \mathrm{D}_{21 / 2}^{0}$ \\
\hline
\end{tabular}


TABLE 2. Wavelengths of Mo II in vacuum - Continued

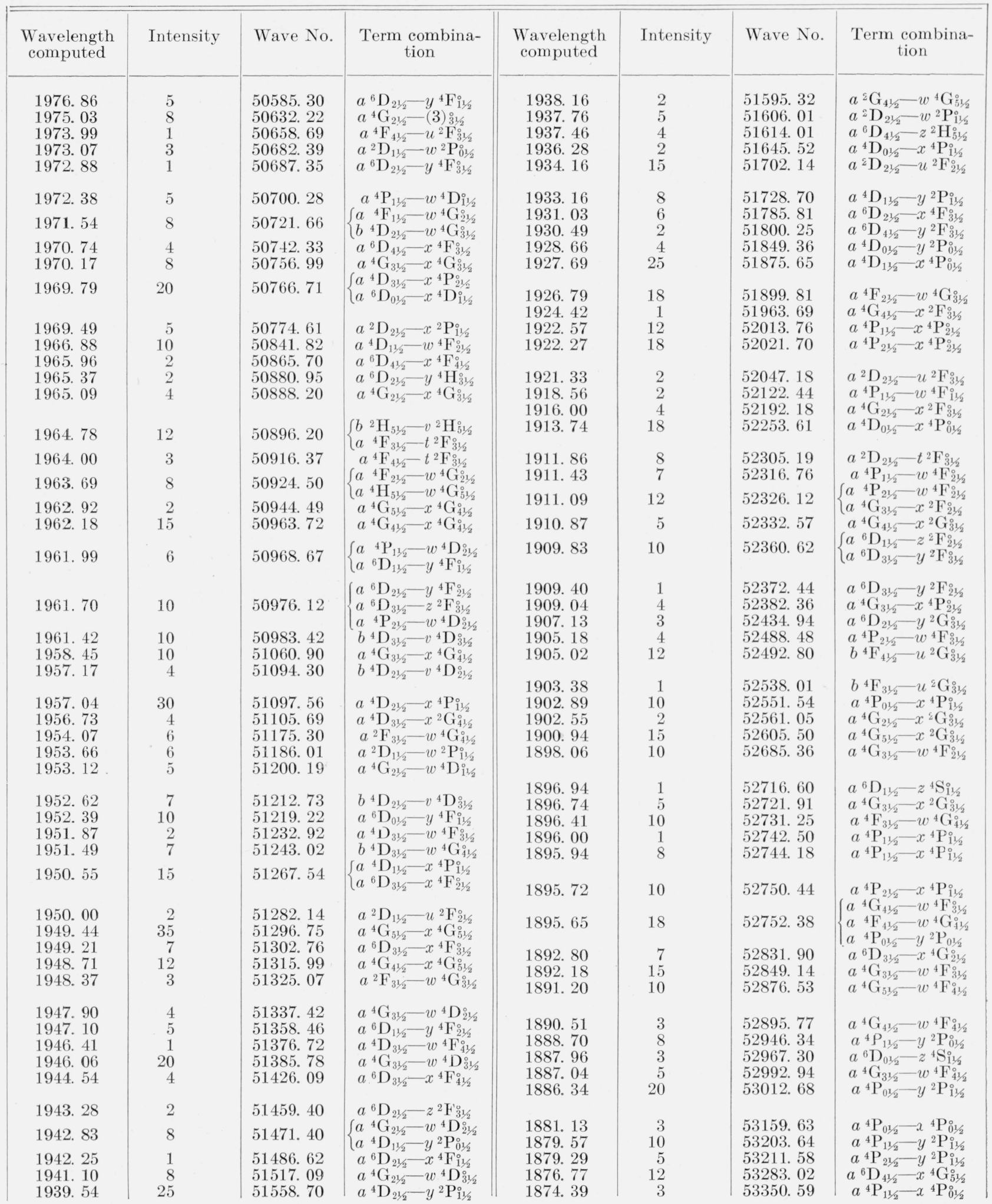


TABLE 2. Wavelengths of Mo II in vacuum-Continued

\begin{tabular}{|c|c|c|c|c|c|c|c|}
\hline $\begin{array}{l}\text { Wavelength } \\
\text { computed }\end{array}$ & Intensity & Wave No. & $\begin{array}{c}\text { Term combina- } \\
\text { tion }\end{array}$ & $\begin{array}{l}\text { Wavelength } \\
\text { computed }\end{array}$ & Intensity & Wave No. & $\begin{array}{c}\text { Term combina- } \\
\text { tion }\end{array}$ \\
\hline $\begin{array}{l}1872.30 \\
1866.79 \\
1865.53 \\
1862.26 \\
1858.66\end{array}$ & $\begin{array}{r}3 \\
1 \\
10 \\
1 \\
18\end{array}$ & $\begin{array}{l}53410.24 \\
53567.94 \\
53604.12 \\
53698.17 \\
53802.28\end{array}$ & $\begin{array}{c}a{ }^{6} \mathrm{D}_{11 / 2}-y^{2} \mathrm{D}_{11 / 2}^{\circ} \\
b^{4} \mathrm{~F}_{31 / 2}-u^{2} \mathrm{G}_{41 / 2}^{\circ} \\
a^{2} \mathrm{I}_{51 / 2}-w^{4} \mathrm{G}_{41 / 2}^{\circ} \\
a^{6} \mathrm{D}_{11 / 2}-x^{4} \mathrm{G}_{21 / 2}^{\circ} \\
a^{2} \mathrm{H}_{41 / 2}-u^{2} \mathrm{G}_{31 / 2}^{\circ}\end{array}$ & $\begin{array}{l}1766.43 \\
1765.51 \\
1764.97 \\
1763.47 \\
1762.10\end{array}$ & $\begin{array}{l}2 \\
1 \\
8 \\
5 \\
3\end{array}$ & $\begin{array}{l}56611.44 \\
56640.85 \\
56658.30 \\
56706.27 \\
56750.61\end{array}$ & $\begin{array}{l}a^{6} \mathrm{D}_{11 / 2}-y^{2} \mathrm{P}_{01 / 2}^{0} \\
a^{6} \mathrm{D}_{41 / 2}-w^{2} \mathrm{~F}_{31 / 2}^{\circ} \\
a^{6} \mathrm{D}_{01 / 2}-x{ }^{4} \mathrm{P}_{11 / 2}^{0} \\
a^{4} \mathrm{D}_{21 / 2}-w^{2} \mathrm{P}_{11 / 2}^{0} \\
a^{4} \mathrm{D}_{01 / 2}-w^{2} \mathrm{P}_{01 / 2}^{11}\end{array}$ \\
\hline $\begin{array}{l}1857.66 \\
1850.80 \\
1843.29 \\
1831.83 \\
1830.96\end{array}$ & $\begin{array}{r}20 \\
3 \\
4 \\
7 \\
6\end{array}$ & $\begin{array}{l}53831.06 \\
54030.74 \\
54250.70 \\
54590.29 \\
54616.08\end{array}$ & $\begin{array}{c}a{ }^{4} \mathrm{~F}_{41 / 2}-w^{4} \mathrm{G}_{51 / 2}^{0} \\
a^{4} \mathrm{P}_{11 / 2}-w^{2} \mathrm{~F}_{21 / 2}^{0} \\
a^{6} \mathrm{D}_{21 / 2}-w^{4} \mathrm{D}_{21 / 2}^{1} \\
a^{6} \mathrm{D}_{01 / 2}-w^{4} \mathrm{D}_{01 / 2}^{\circ} \\
a^{6} \mathrm{D}_{11 / 2}-w^{4} \mathrm{D}_{11 / 2}^{\circ}\end{array}$ & $\begin{array}{l}1759.33 \\
1758.64 \\
1758.43 \\
1753.90 \\
1750.72\end{array}$ & $\begin{array}{r}15 \\
15 \\
12 \\
15 \\
4\end{array}$ & $\begin{array}{l}56839.75 \\
56862.14 \\
56868.74 \\
57015.69 \\
57119.44\end{array}$ & $\begin{array}{l}a^{4} \mathrm{G}_{21 / 2}-w^{2} \mathrm{D}_{11 / 2}^{\circ} \\
a^{6} \mathrm{D}_{01 / 2}-y^{2} \mathrm{P}_{01 / 2}^{\circ} \\
a^{6} \mathrm{D}_{11 / 2}-y^{2} \mathrm{P}_{11 / 2}^{\circ} \\
a^{6} \mathrm{D}_{11 / 2}-x^{4} \mathrm{P}_{01 / 2}^{\circ} \\
a^{6} \mathrm{D}_{01 / 2}-y^{2} \mathrm{P}_{11 / 2}^{\circ}\end{array}$ \\
\hline $\begin{array}{l}\text { 1829. } 04 \\
1828.71 \\
1825.08 \\
1823.75 \\
1822.66\end{array}$ & $\begin{array}{l}5 \\
4 \\
5 \\
7 \\
4\end{array}$ & $\begin{array}{l}54673.55 \\
54683.44 \\
54792.20 \\
54832.21 \\
54864.90\end{array}$ & 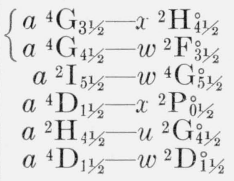 & $\begin{array}{l}1750.14 \\
1747.78 \\
1746.22 \\
1739.16 \\
1738.53\end{array}$ & $\begin{array}{r}10 \\
1 \\
22 \\
6 \\
1\end{array}$ & $\begin{array}{l}57138.40 \\
57215.32 \\
57266.39 \\
57499.06 \\
57519.81\end{array}$ & $\begin{array}{c}a{ }^{4} \mathrm{P}_{21 / 2}-w^{2} \mathrm{D}_{21 / 2}^{\circ} \\
b{ }^{4} \mathrm{D}_{31 / 2}-u^{2} \mathrm{G}_{41 / 2} \\
a^{6} \mathrm{D}_{01 / 2}-x^{4} \mathrm{P}_{01 / 2}^{\circ} \\
a^{4} \mathrm{G}_{31 / 2}-w^{2} \mathrm{D}_{21 / 2}^{2} \\
a^{4} \mathrm{P}_{11 / 2}-x^{2} \mathrm{P}_{11 / 2}^{\circ}\end{array}$ \\
\hline $\begin{array}{l}1821.42 \\
1820.50 \\
1813.56 \\
1812.57 \\
1811.07\end{array}$ & $\begin{array}{r}8 \\
12 \\
2 \\
20 \\
6\end{array}$ & $\begin{array}{l}54902.30 \\
54929.90 \\
55140.09 \\
55170.16 \\
55215.85\end{array}$ & $\begin{array}{l}a^{4} \mathrm{G}_{21 / 2}-w^{2} \mathrm{~F}_{31 / 2}^{\circ} \\
a^{2} \mathrm{H}_{51 / 2}-u^{2} \mathrm{G}_{41 / 2}^{\circ} \\
a^{4} \mathrm{D}_{21 / 2}-v^{2} \mathrm{~F}_{21 / 2}^{0} \\
a^{4} \mathrm{D}_{01 / 2}-x^{2} \mathrm{P}_{01 / 2}^{0} \\
a^{6} \mathrm{~S}_{21 / 2}-z^{4} \mathrm{D}_{21 / 2}^{0}\end{array}$ & $\begin{array}{l}\text { 1735. } 20 \\
1734.41 \\
1733.90 \\
1731.54 \\
1730.02 \\
1707.25\end{array}$ & $\begin{array}{r}4 \\
3 \\
1 \\
50 \\
2\end{array}$ & $\begin{array}{l}57630.37 \\
57656.63 \\
57673.62 \\
57752.07 \\
57802.77\end{array}$ & $\begin{array}{l}a^{4} \mathrm{G}_{21 / 2}-w^{2} \mathrm{D}_{21 / 2}^{\circ} \\
a^{4} \mathrm{P}_{01 / 2}-w^{2} \mathrm{P}_{01 / 2}^{\circ} \\
a^{4} \mathrm{~F}_{31 / 2}-u^{2} \mathrm{G}_{31 / 2}^{0} \\
a^{4} \mathrm{D}_{11 / 2}-t^{2} \mathrm{~F}_{21 / 2}^{1} \\
a^{4} \mathrm{D}_{31 / 2}-t^{2} \mathrm{~F}_{31 / 2}^{0}\end{array}$ \\
\hline $\begin{array}{l}\text { 1810. } 82 \\
1802.27 \\
1801.73 \\
1795.12 \\
1789.71\end{array}$ & $\begin{array}{r}10 \\
5 \\
4 \\
6 \\
6\end{array}$ & $\begin{array}{l}55223.60 \\
55485.52 \\
55502.31 \\
55706.57 \\
55874.87\end{array}$ & $\begin{array}{c}a^{4} \mathrm{G}_{51 / 2}-x^{2} \mathrm{H}_{51 / 2}^{\circ} \\
a^{4} \mathrm{D}_{21 / 2}-w^{2} \mathrm{D}_{21 / 2}^{\circ} \\
a^{4} \mathrm{P}_{21 / 2}-w^{2} \mathrm{G}_{31 / 2}^{\circ} \\
a^{6} \mathrm{~S}_{2112}-z^{4} \mathrm{D}_{31 / 2}^{0} \\
a^{4} \mathrm{D}_{21 / 2}-x^{2} \mathrm{P}_{11 / 2}^{\circ}\end{array}$ & $\begin{array}{l}1727.35 \\
1724.75 \\
1713.76 \\
1693.39 \\
1681.29\end{array}$ & $\begin{array}{l}5 \\
3 \\
1 \\
3 \\
2\end{array}$ & $\begin{array}{l}57892.05 \\
57979.39 \\
58351.21 \\
59053.32 \\
59478.34\end{array}$ & $\begin{array}{l}a{ }^{6} \mathrm{~S}_{21 / 2}-z^{4} \mathrm{H}_{31 / 2}{ }^{0} \\
a{ }^{4} \mathrm{D}_{31 / 2}-t^{2} \mathrm{~F}_{21 / 2}^{2} \\
a{ }^{4} \mathrm{P}_{11 / 2}-w^{2} \mathrm{P}_{11 / 2}^{0} \\
a{ }^{6} \mathrm{~S}_{21 / 2}-z^{4} \mathrm{G}_{21 / 2}^{1} \\
a{ }^{6} \mathrm{~S}_{21 / 2}-z^{4} \mathrm{G}_{31 / 2}^{0}\end{array}$ \\
\hline $\begin{array}{l}1789.09 \\
1784.94 \\
1783.29 \\
1782.99 \\
1780.98\end{array}$ & $\begin{array}{r}15 \\
4 \\
2 \\
18 \\
25\end{array}$ & $\begin{array}{l}55994.28 \\
56024.38 \\
56076.18 \\
56085.50 \\
56148.88\end{array}$ & $\begin{array}{c}a^{4} \mathrm{G}_{21 / 2}-w^{2} \mathrm{G}_{31 / 2}^{\circ} \\
a^{6} \mathrm{D}_{21 / 2}-x^{4} \mathrm{P}_{11 / 2}^{\circ} \\
a^{4} \mathrm{P}_{01 / 2}-x^{2} \mathrm{P}_{01 / 2}^{0} \\
a^{4} \mathrm{D}_{31 / 2}-v^{2} \mathrm{~F}_{31 / 2}^{\circ} \\
a^{4} \mathrm{P}_{01 / 2}-w^{2} \mathrm{D}_{11 / 2}^{\circ}\end{array}$ & $\begin{array}{l}1674.29 \\
1668.63 \\
1610.97 \\
\text { 1605. } 99 \\
1603.24\end{array}$ & $\begin{array}{r}1 \\
3 \\
12 \\
5 \\
4\end{array}$ & $\begin{array}{l}\text { 59726. } 92 \\
59929.29 \\
62074.26 \\
62266.77 \\
62373.67\end{array}$ & $\begin{array}{l}a^{4} \mathrm{G}_{21 / 2}-t^{2} \mathrm{~F}_{21 / 2}^{\circ} \\
a^{6} \mathrm{D}_{31 / 2}-w^{2} \mathrm{D}_{21 / 2}^{\circ} \\
a^{6} \mathrm{D}_{21 / 2}-u^{2} \mathrm{~F}_{31 / 2}^{\circ} \\
a^{6} \mathrm{D}_{01 / 2}-w^{2} \mathrm{P}_{11 / 2}^{11} \\
a^{6} \mathrm{D}_{41 / 2}-w^{4} \mathrm{G}_{31 / 2}^{\circ}\end{array}$ \\
\hline $\begin{array}{l}1773.91 \\
1772.81 \\
1772.59 \\
1772.33 \\
1770.37\end{array}$ & $\begin{array}{l}2 \\
3 \\
3 \\
3 \\
8\end{array}$ & $\begin{array}{l}56372.65 \\
56407.60 \\
56414.70 \\
56422.83 \\
56485.52\end{array}$ & $\begin{array}{l}a^{4} \mathrm{D}_{11 / 2}-w^{2} \mathrm{P}_{01 / 2}^{\circ} \\
a^{6} \mathrm{D}_{11 / 2}-x{ }^{4} \mathrm{P}_{11 / 2} \\
b^{4} \mathrm{D}_{21 / 2}-u^{2} \mathrm{G}_{31 / 2} \\
a^{4} \mathrm{D}_{01 / 2}-x{ }^{2} \mathrm{P}_{11 / 2}^{0} \\
a^{6} \mathrm{D}_{21 / 2}-y{ }^{2} \mathrm{P}_{11 / 2}^{\circ}\end{array}$ & $\begin{array}{l}1591.73 \\
1586.99 \\
1552.90\end{array}$ & $\begin{array}{l}2 \\
5 \\
3\end{array}$ & $\begin{array}{l}\text { 62824. } 80 \\
63012.24 \\
64395.44\end{array}$ & $\begin{array}{c}a^{6} \mathrm{D}_{11 / 2}-w^{4} \mathrm{G}_{21 / 2}^{1} \\
a^{6} \mathrm{~S}_{21 / 2}-(2)_{31 / 2}^{\circ} \\
a^{6} \mathrm{~S}_{21 / 2}-z^{2} \mathrm{~F}_{21 / 2}^{2}\end{array}$ \\
\hline
\end{tabular}

TABLE 3. Zeeman effect of Mo II

\begin{tabular}{|c|c|c|c|c|c|}
\hline $\begin{array}{l}\text { Wave- } \\
\text { lengths }\end{array}$ & Magnetic patterns & $\begin{array}{l}\text { Wave- } \\
\text { lengths }\end{array}$ & Magnetic patterns & $\begin{array}{l}\text { Wave- } \\
\text { lengths }\end{array}$ & Magnetic patterns \\
\hline $\begin{array}{l}4667.40 \\
4637.95 \\
4604.20 \\
4565.69 \\
4543.40 \\
\\
4553.50 \\
4519.60 \\
4452.02 \\
4433.49 \\
4407.38\end{array}$ & $\begin{array}{l}(\mathbf{0 . 0 9 1}, 0.269) \mathbf{0 . 7 8 7}, 0.964 \\
1.138 \\
(0.000 W) 1.139 A \\
(0.000 W) 1.230 A \\
(0.094) 0.837 \\
(0.000) 1.200 \\
\\
(0.364) \mathbf{1 . 4 5 9}, 2.186 \\
(0.075) 1.597 \\
(0.000) 1.07 \dagger \\
(0.00) 0.97 \dagger \\
(0.00) 1.10 \dagger \\
(0.00) 1.41 \dagger \\
(0.00 w) 1.62 B \dagger \\
(0.00) 1.06 \dagger \\
(0.804) \mathbf{0 . 3 9 6 ,} 1.993 \\
(0.21) 1.01 \dagger\end{array}$ & $\begin{array}{l}\text { 4403. } 29 \\
4388.26 \\
4377.75 \\
4363.64 \\
\text { 4361. } 91 \\
\text { 4358. } 27 \\
\text { 4356. } 10 \\
4328.01 \\
\text { 4315. } 23 \\
\text { 4311. } 65\end{array}$ & $\begin{array}{l}(0.28) 1.02 \dagger \\
(0.00) 1.06 \dagger \\
(1.374) 1.422 \\
(\mathbf{0 . 2 8 4}, 0.837) \mathbf{0 . 4 2 0}, 0.992, \\
1.547,2.093 \\
(0.000) 1.111 \\
(0.000 w) 0.856 A \\
(0.00) 1.50 \dagger \\
(\mathbf{0 . 1 3 6}, 0.403) 0.955,1.207, \\
\mathbf{1 . 4 6 5} \\
(\mathbf{0 . 2 1 7 ,}, 0.682,1.137) \mathbf{0 . 2 2 7}, \\
0.709,1.144 \\
(\mathbf{0 . 3 8 8 ,} 1.145)-\mathbf{0 . 0 8 0}, \\
0.689,1.430,2.180 \\
(0.000) 1.038 \\
(0.300, \mathbf{0 . 9 4 3}) 0.877, \mathbf{1 . 5 1 4}, \\
2.140\end{array}$ & $\begin{array}{l}\text { 4274. } 44 \\
4250.69 \\
4246.58 \\
4244.72 \\
4227.07 \\
4209.65 \\
\text { 4192. } 27 \\
\text { 4191. } 03 \\
\text { 4177. } 94 \\
4171.79 \\
4161.25 \\
4150.82 \\
4146.87\end{array}$ & $\begin{array}{l}(0.000) 1.108 \\
(\mathbf{0 . 1 7 6}, 0.546,0.905) \mathbf{0 . 4 6 2}, \\
0.825,1.198,1.562,1.928 \\
(0.00) 1.61 \dagger \\
(0.000) 0.861 \\
(0.883) 0.941, \mathbf{2 . 6 8 9} \\
(0.269,0.740, \mathbf{1 . 2 2 9}) 0.535, \\
1.021, \mathbf{1 . 5 1 2}, 2.004,2.487 \\
(\mathbf{0 . 0 7 3}, 0.222,0.359) 0.947, \\
1.089,1.224, \mathbf{1 . 3 6 8} \\
(?) 0.84 \dagger \\
(\mathbf{0 . 5 5 0 ,} 1.595)-\mathbf{0 . 3 1 9}, \\
+0.737 \\
(0.000 w) 0.960 w \\
(1.035, \mathbf{1 . 7 3 1}) 0.000,0.701, \\
\mathbf{1 . 4 0 0 ,} 2.113,2.800 \\
(0.000) 1.097 \\
(0.239) \mathbf{0 . 9 4 2}, 1.421\end{array}$ \\
\hline
\end{tabular}


TABLE 3. Zeeman effect of Mo II-Continued

\begin{tabular}{|c|c|c|c|c|c|}
\hline $\begin{array}{l}\text { Wave- } \\
\text { lengths }\end{array}$ & Magnetic patterns & $\begin{array}{l}\text { Wave- } \\
\text { lengths }\end{array}$ & Magnetic patterns & $\begin{array}{l}\text { Wave- } \\
\text { lengths }\end{array}$ & Magnetic patterns \\
\hline $\begin{array}{l}4077.68 \\
4058.61 \\
4038.81 \\
4029.96 \\
4023.54\end{array}$ & 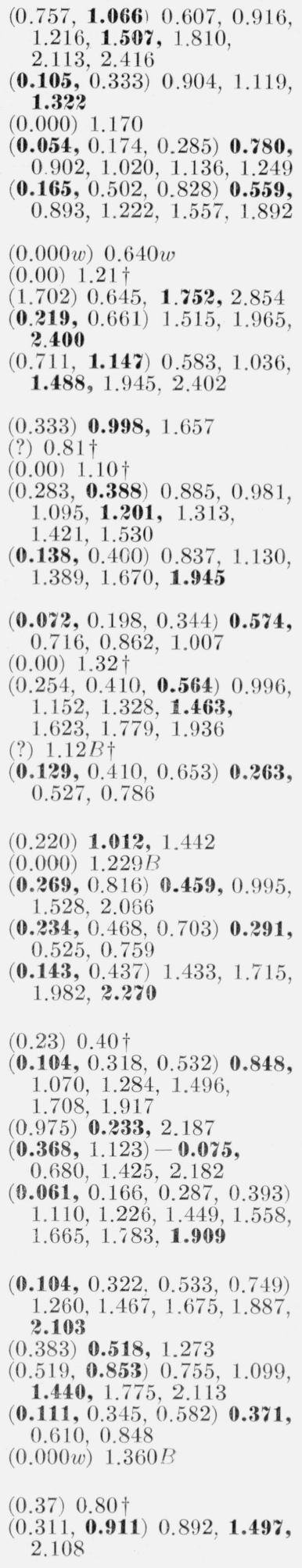 & $\begin{array}{l}3908.61 \\
3906.54 \\
3904.96 \\
\\
3882.31 \\
3871.90 \\
3871.45 \\
3866.88 \\
3861.29\end{array}$ & 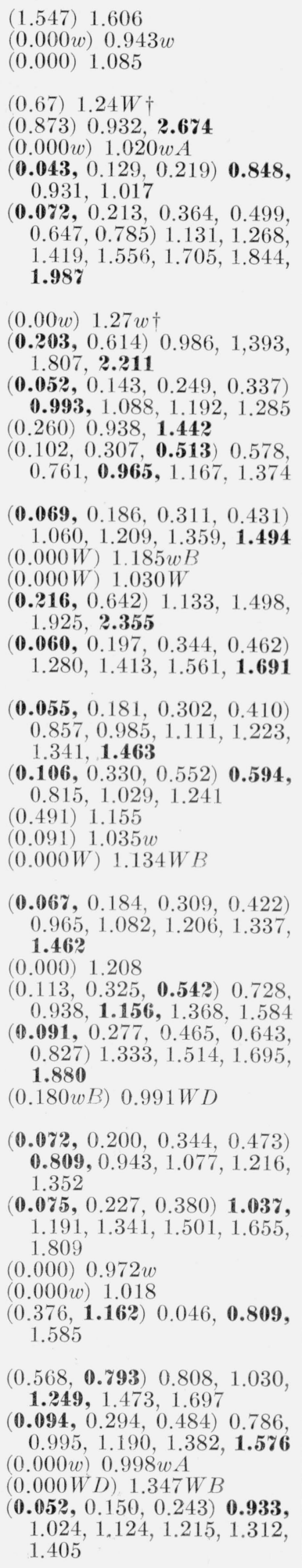 & $\begin{array}{l}3627.35 \\
3625.54 \\
\\
3623.68 \\
3622.84 \\
3620.18 \\
3618.36\end{array}$ & 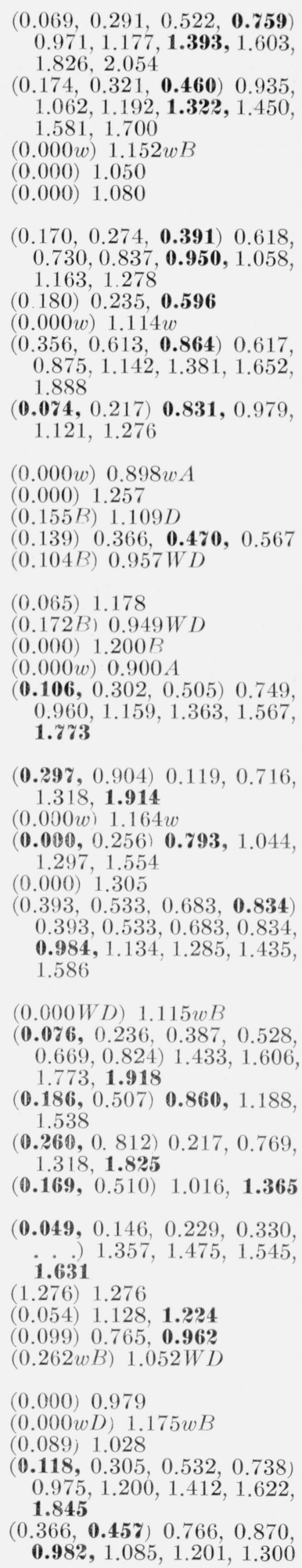 \\
\hline
\end{tabular}


Table 3. Zeeman effect of Mo II-Continued

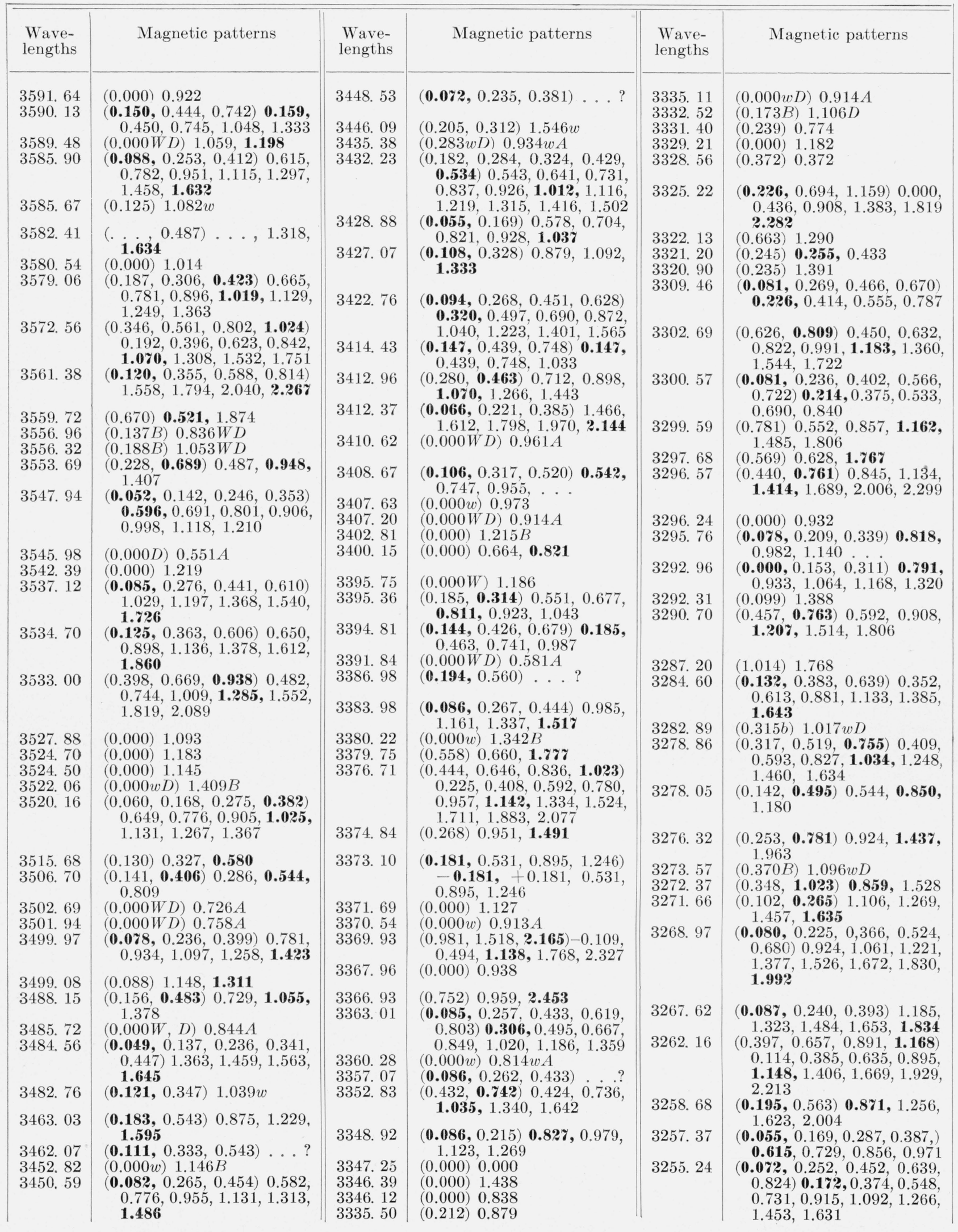


TABle 3. Zeeman effect of Mo II-Continued

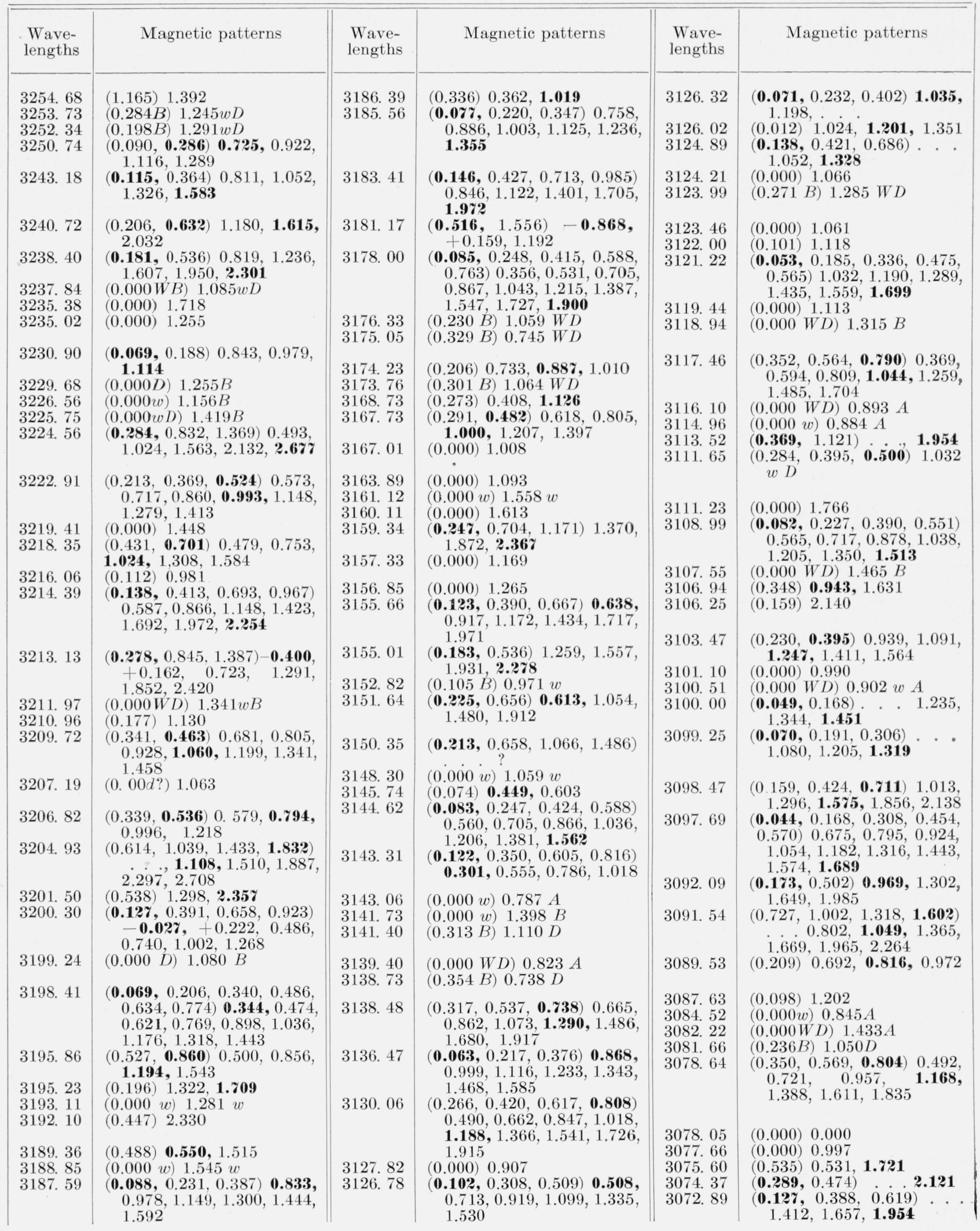


TABLE 3. Zeeman effect of Mo II-Continued

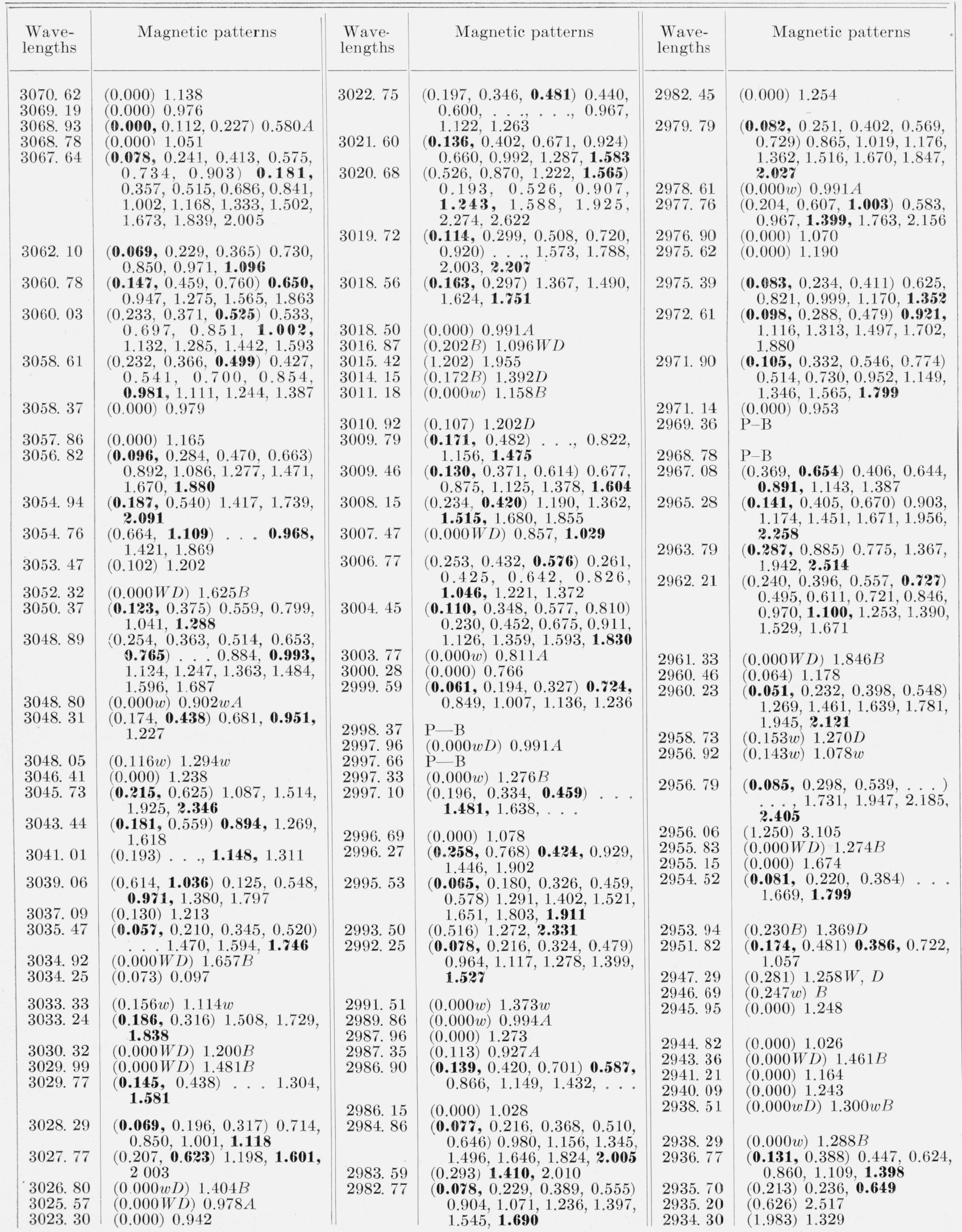


TABLE 3. Zeeman effect of Mo II-Continued

\begin{tabular}{|c|c|c|c|c|c|}
\hline $\begin{array}{l}\text { Wave- } \\
\text { lengths }\end{array}$ & Magnetic patterns & $\begin{array}{l}\text { Wave- } \\
\text { lengths }\end{array}$ & Magnetic patterns & $\begin{array}{l}\text { Wave- } \\
\text { lengths }\end{array}$ & Magnetic patterns \\
\hline $\begin{array}{l}2892.82 \\
2892.04 \\
2891.29 \\
2890.99 \\
2888.70 \\
2888.17 \\
2886.98\end{array}$ & 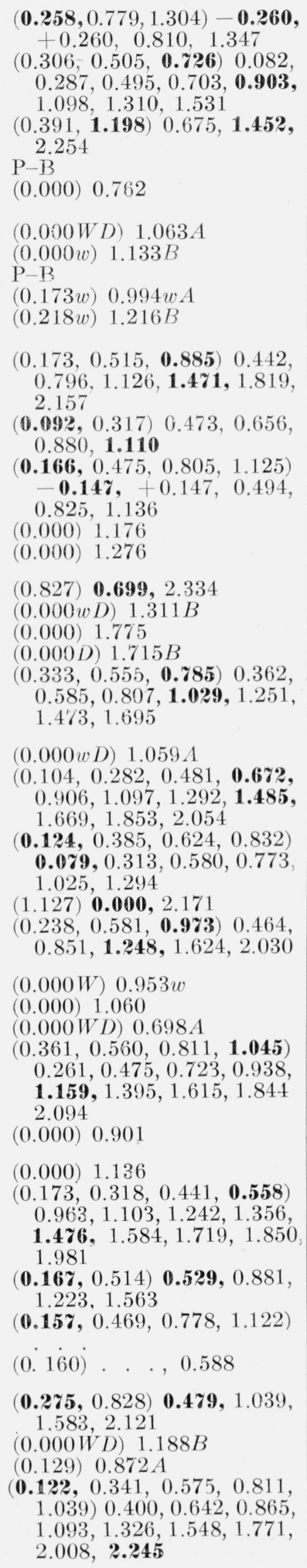 & $\begin{array}{l}2863.80 \\
2863.20 \\
\\
2859.00 \\
\\
2858.08 \\
2856.90 \\
2856.01 \\
2855.71\end{array}$ & 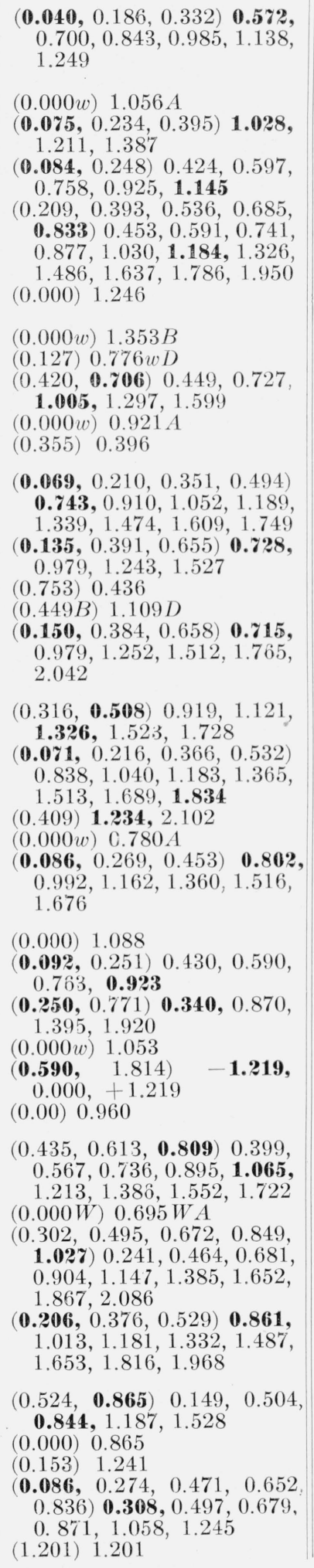 & $\begin{array}{l}2827.75 \\
2827.18 \\
2825.30 \\
2824.19 \\
2822.94 \\
2822.04 \\
2821.84 \\
2821.02 \\
2820.02 \\
2819.59 \\
2817.54 \\
2817.44 \\
2816.16 \\
2815.00\end{array}$ & 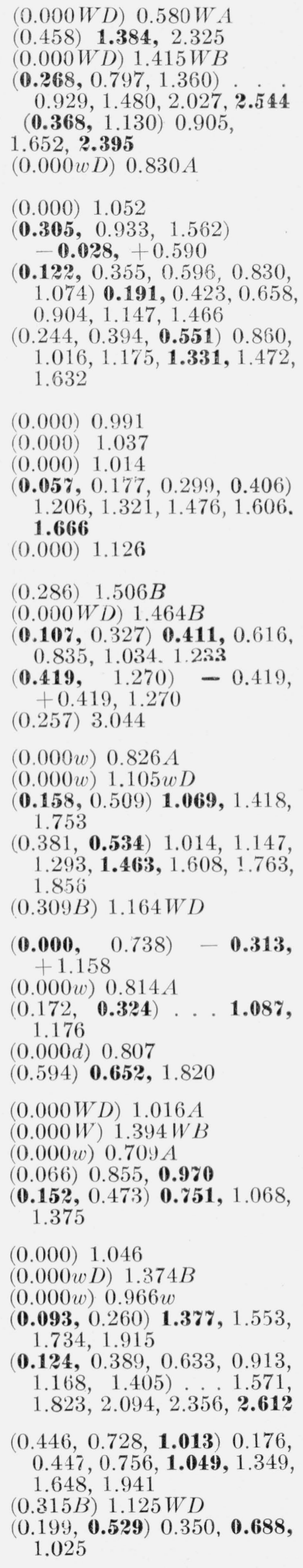 \\
\hline
\end{tabular}


TABle 3. Zeeman effect of Mo II-Continued

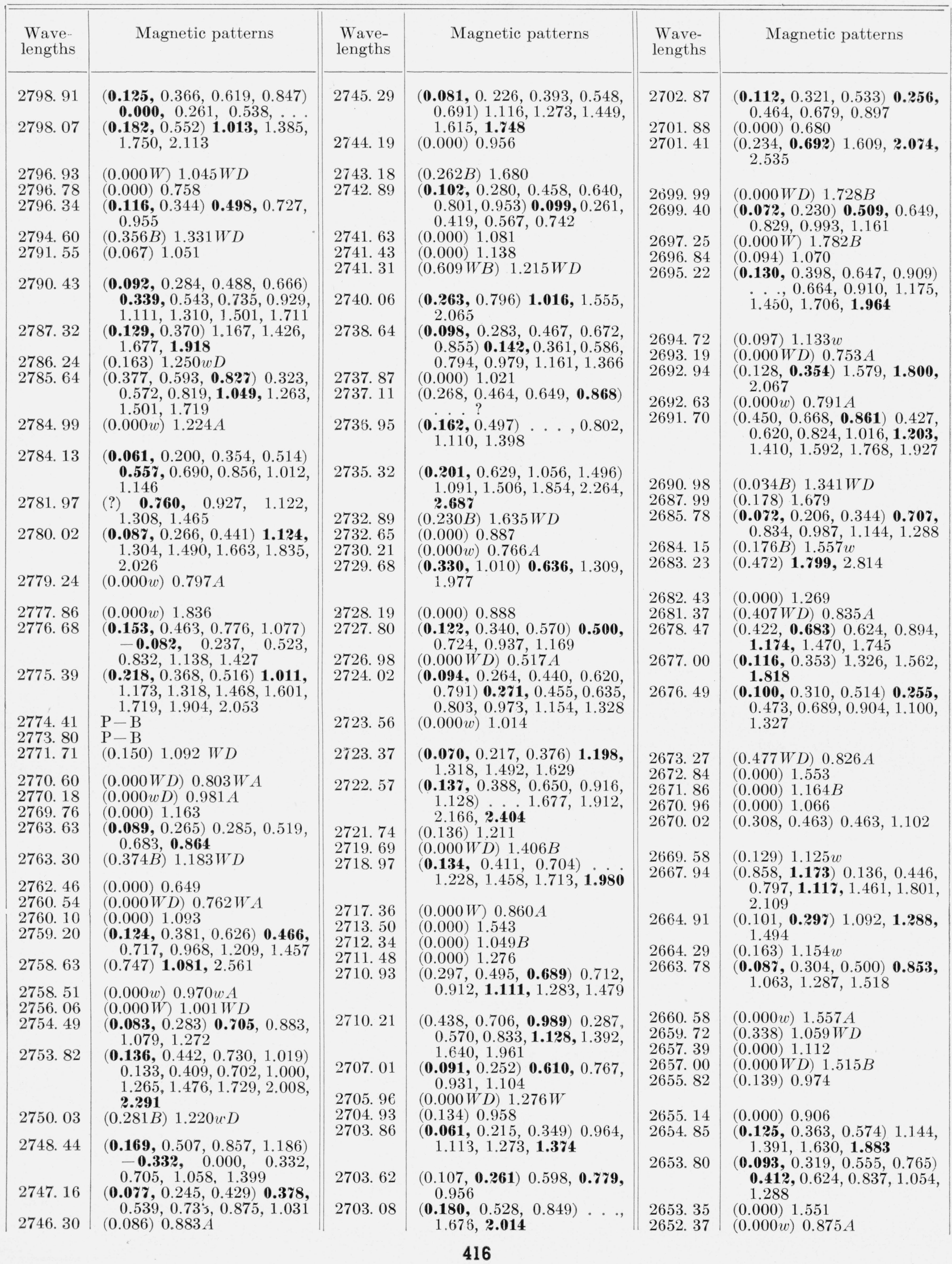


TABLE 3. Zeeman effect of Mo II-Continued

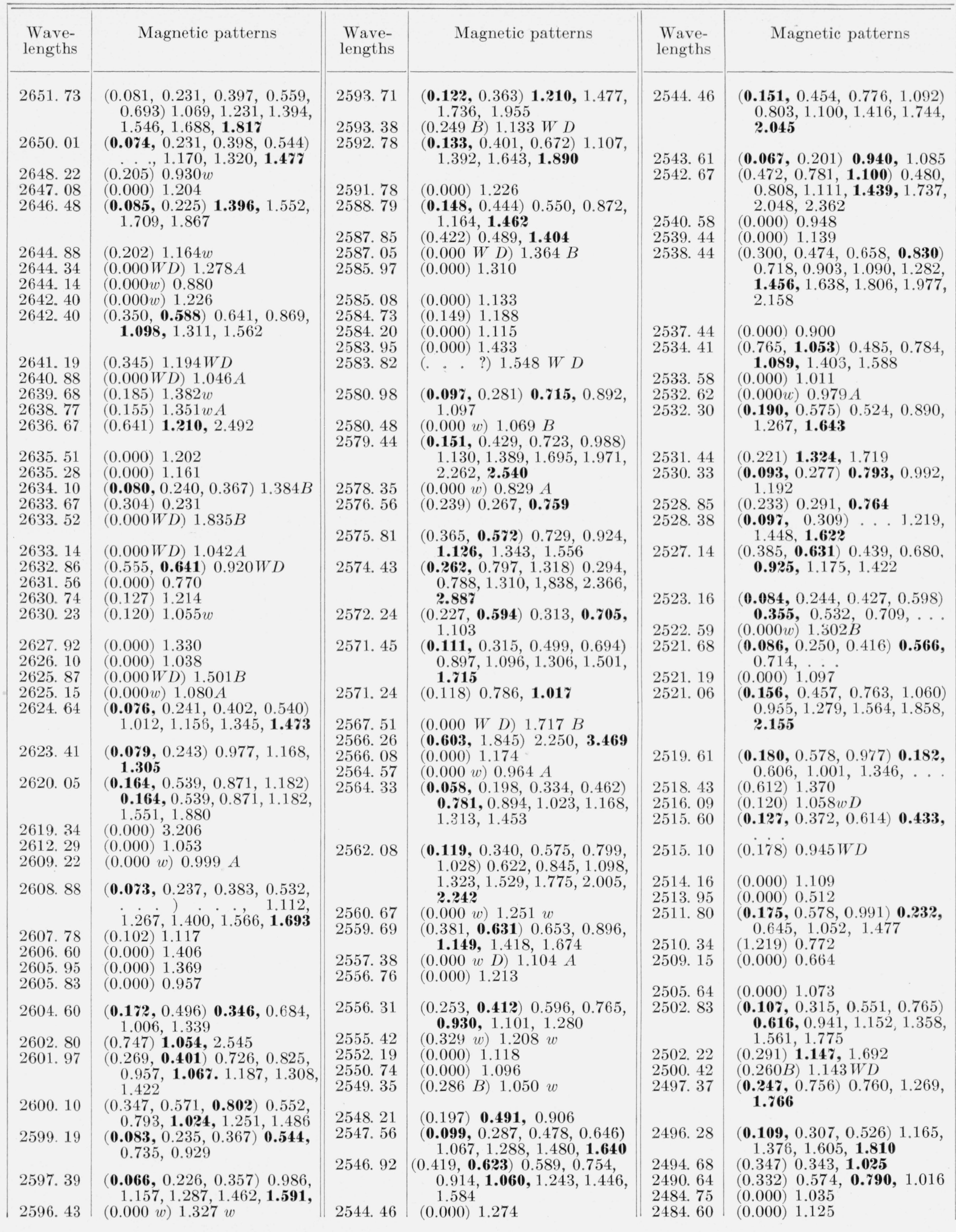


TABLE 3. Zeeman effect of Mo II-Continued

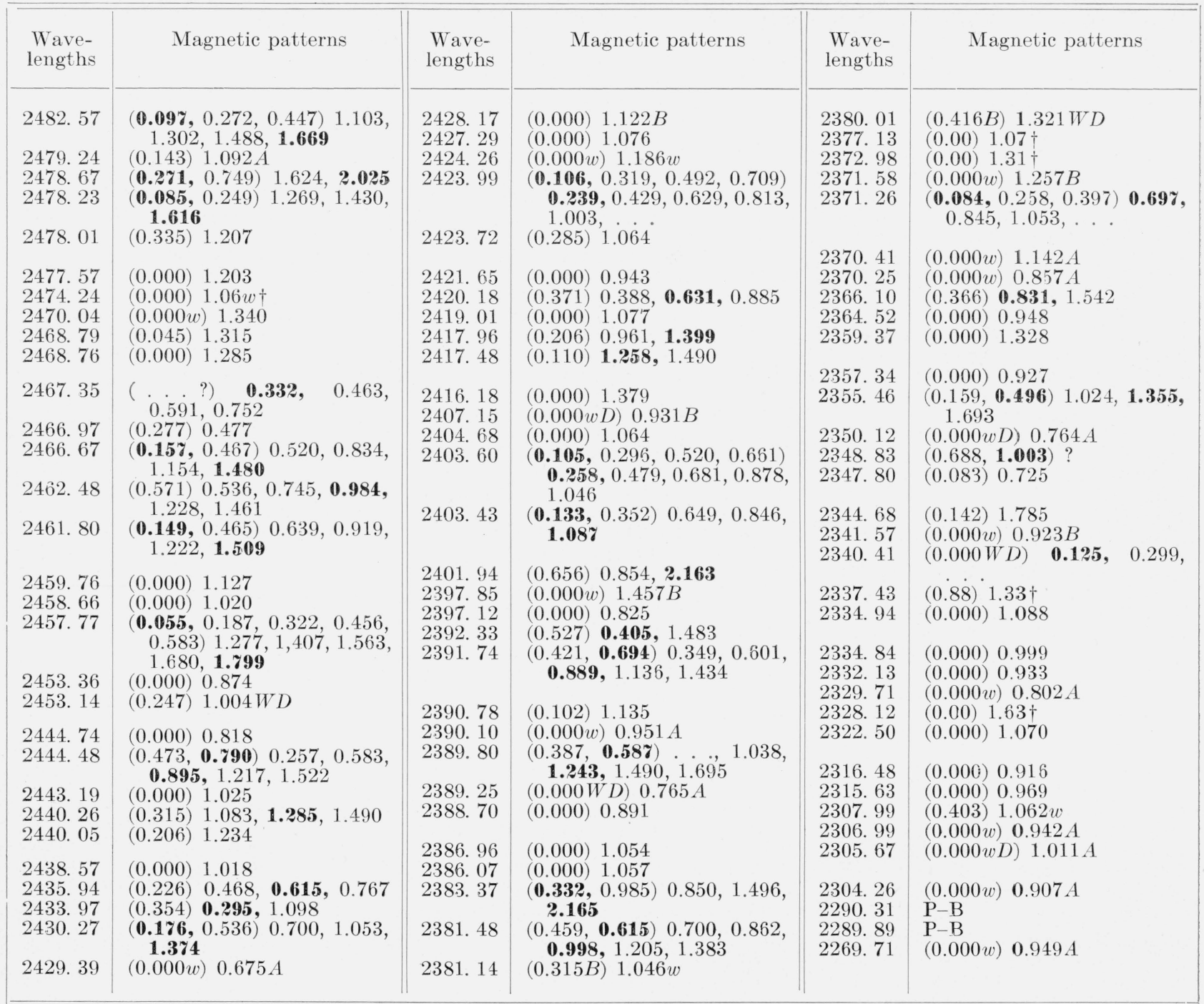

TABLE 4. Even terms of Mo II

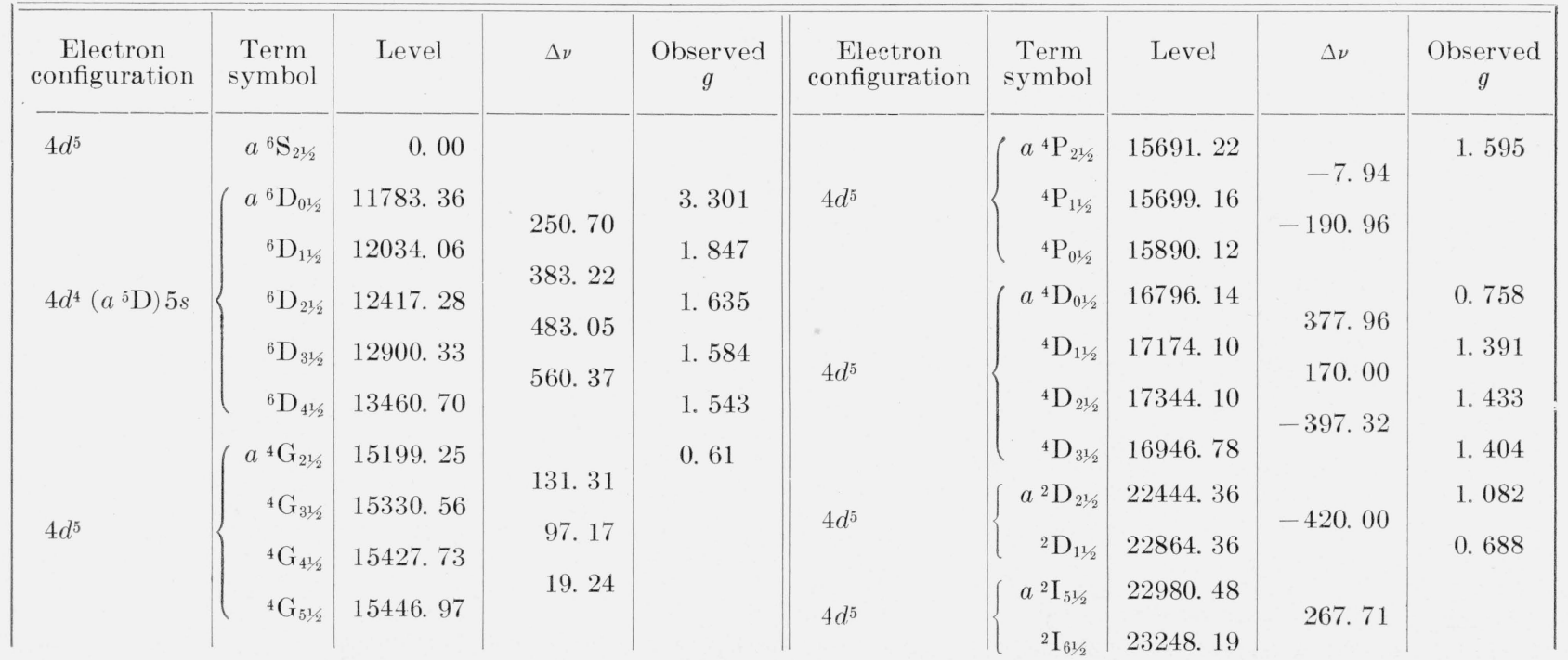


TABLE 4. Even terms of Mo II-Continued

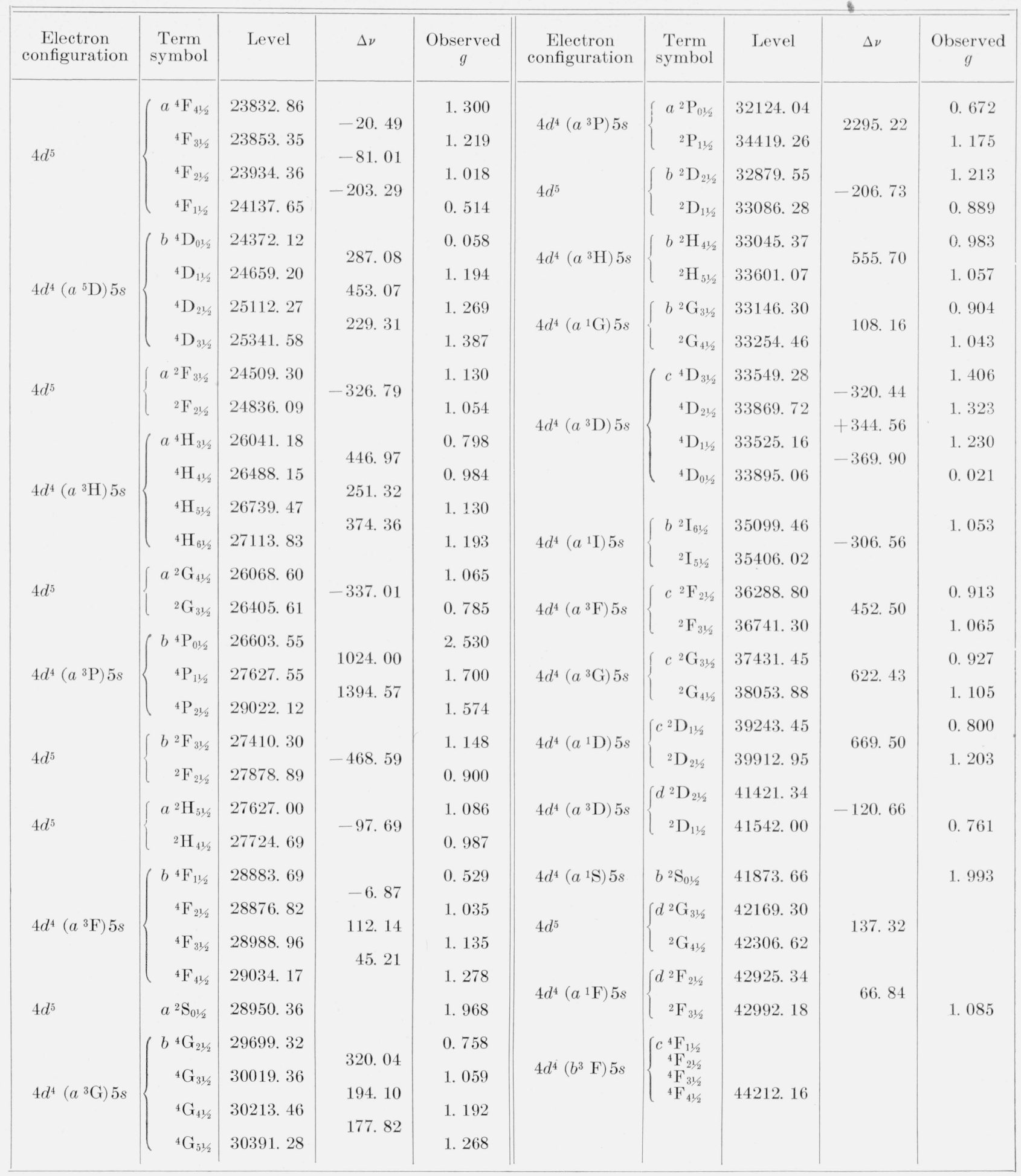


TABLE 5. Odd terms of Mo II

\begin{tabular}{|c|c|c|c|c|c|c|c|c|c|}
\hline $\begin{array}{c}\text { Electron } \\
\text { configuration }\end{array}$ & $\begin{array}{l}\text { Term } \\
\text { symbol }\end{array}$ & Level & $\Delta \nu$ & $\underset{g}{\text { Observed }}$ & $\begin{array}{l}\text { Electron } \\
\text { configuration }\end{array}$ & $\begin{array}{l}\text { Term } \\
\text { symbol }\end{array}$ & Level & $\Delta \nu$ & $\begin{array}{c}\text { Observed } \\
g\end{array}$ \\
\hline \multirow{6}{*}{$4 d^{4}\left(a^{5} \mathrm{D}\right) 5 p$} & $\left(z^{6} \mathrm{~F}_{01 / 2}^{\circ}\right.$ & 45853. 08 & 205 & -0.650 & \multirow{5}{*}{$4 d^{4}\left(a^{3} \mathrm{~F}\right) 5 p$} & $\left(z^{4} \mathrm{G}_{21 / 2}^{\circ}\right.$ & 59053.32 & \multirow{3}{*}{$\begin{array}{l}425.02 \\
748.66 \\
889.18\end{array}$} & 0.672 \\
\hline & ${ }^{6} \mathrm{~F}_{11 / 2}$ & 46148. 12 & & 1. 072 & & ${ }^{4} \mathrm{G}_{31 / 2}^{\circ}$ & 59478.34 & & 0.923 \\
\hline & ${ }^{6} \mathrm{~F}_{21 / 2}^{\circ}$ & 46614. 14 & 61784 & 1. 305 & & ${ }^{4} \mathrm{G}_{41 / 2}^{\circ}$ & 60227.00 & & 0.884 \\
\hline & ${ }^{6} \mathrm{~F}_{31 / 2}^{0}$ & 47231. 98 & & 1. 375 & & ${ }^{4} \mathrm{G}_{51 / 2}^{\circ}$ & 61116. 18 & & 1. 210 \\
\hline & ${ }^{6} \mathrm{~F}_{41 / 2}^{\circ}$ & 47999.47 & 960.21 & 1. 415 & & & 59679.75 & \multirow{4}{*}{$\begin{array}{r}1244.78 \\
723.23 \\
503.90\end{array}$} & 1. 027 \\
\hline & ${ }^{6} \mathrm{~F}_{51 / 2}^{\circ}$ & 48959. 68 & & & \multirow{3}{*}{$4 d^{4}\left(a^{3} \mathrm{H}\right) 5 p$} & ${ }^{4} I_{51 / 2}^{\circ}$ & 60924.53 & & 0. 936 \\
\hline \multirow{3}{*}{$4 d^{4}\left(a^{5} \mathrm{D}\right) 5 p$} & $z^{4} \mathrm{P}_{01 / 2}^{\circ}$ & 47208. 36 & 814.09 & 2. 779 & & & 61647. 76 & & 1. 103 \\
\hline & ${ }^{4} \mathrm{P}_{11 / 2}^{\circ}$ & 48022.45 & & 1. 818 & & ${ }^{4} I_{71 / 2}^{\circ}$ & 62151.66 & & \\
\hline & ${ }^{4} \mathrm{P}_{21 / 2}^{\circ}$ & 48860.57 & (2) & 1. 742 & \multirow{2}{*}{$4 d^{4}\left(a^{3} \mathrm{~F}\right) 5 p$} & & & \multirow{2}{*}{1151.77} & 0.862 \\
\hline \multirow{3}{*}{$4 d^{4}\left(a^{5} \mathrm{D}\right) 5 p$} & $\left(z^{6} \mathrm{P}_{11 / 2}\right.$ & 49040.82 & 567.92 & 2. 305 & & ${ }^{2} \mathrm{D}_{21 / 2}^{\circ}$ & 60992.47 & & 1. 205 \\
\hline & & 49608. 74 & -127.70 & 1. 718 & \multirow{5}{*}{$4 d^{4}\left(a^{3} \mathrm{P}\right) 5 p$} & & 60135. 37 & \multirow{2}{*}{837.77} & 1. 011 \\
\hline & ${ }^{6} \mathrm{P}_{31 / 2}^{\circ}$ & 49481. 04 & & 1. 672 & & ${ }^{2} \mathrm{G}_{\mathfrak{4} 1 / 2}$ & 60973. 14 & & 1. 101 \\
\hline \multirow{5}{*}{$4 d^{4}\left(a_{-}^{-5} \mathrm{D}\right) 5 p$} & & 49949. 45 & 242.55 & 3. 155 & & $\left(y^{4} \mathrm{P}_{01 / 2}^{\circ}\right.$ & 61134. 25 & 22259 & 2. 299 \\
\hline & ${ }^{6} \mathrm{D}_{1 / 2}^{\circ}$ & 50192.00 & 385. 36 & 1. 802 & & ${ }^{4} \mathrm{P}_{11 / 2}^{\circ}$ & 61456. 77 & 322.52 & 1. 656 \\
\hline & ${ }^{6} \mathrm{D}_{21 / 2}^{\circ}$ & & -274.82 & 1. 597 & & ${ }^{4} \mathrm{P}_{21 / 2}^{\circ}$ & 62425.47 & 908.10 & 1. 185 \\
\hline & ${ }^{6} \mathrm{D}_{31 / 2}^{\circ}$ & 50302.54 & 402. 98 & 1. 552 & & $\int z^{2} \mathrm{P}_{11 / 2}^{\circ}$ & 61746.58 & & 1. 220 \\
\hline & ${ }^{6} \mathrm{D}_{41 / 2}^{\circ}$ & 50705.52 & & 1. 502 & $4 d^{4}(c$ & & 62096. 05 & -349.47 & 1. 075 \\
\hline \multirow{4}{*}{$4 d^{4}\left(a_{-}^{5} \mathrm{D}\right) 5 p$} & $\left(z^{4} \mathrm{~F}_{11 / 2}^{\circ}\right.$ & 51372.90 & 359.49 & 0.412 & \multirow{4}{*}{$4 d^{4}\left(a^{3} \mathrm{~F}\right) 5 p$} & $\left(x^{4} \mathrm{D}_{31 / 2}^{\circ}\right.$ & 62491.78 & & 1. 252 \\
\hline & & 51732. 39 & 484. 91 & 1. 045 & & ${ }^{4} \mathrm{D}_{21 / 2}^{\circ}$ & 62342.27 & 149.51 & 1. 067 \\
\hline & ${ }^{4} \mathrm{~F}_{31 / 2}^{\circ}$ & 52217.30 & 62580 & 1. 262 & & & 62551.04 & -208.77 & 0.707 \\
\hline & & 52843. 10 & & 1. 362 & & & & -386.41 & 0.030 \\
\hline \multirow{3}{*}{$4 d^{4}\left(a^{5} \mathrm{D}\right) 5 p$} & $\left(z^{4} \mathrm{D}_{01 / 2}^{1 / 2}\right.$ & 54238.80 & 448. 81 & 0. 042 & \multirow[b]{3}{*}{$4 d^{4}\left(a^{3} \mathrm{H}\right) 5 p$} & & 62594.53 & \multirow[b]{3}{*}{ 251. 89} & 1. 14 \\
\hline & & 34006 & 528. 24 & & & $\left(z^{2} I_{51 / 2}^{\circ}\right.$ & 62728.35 & & 0.902 \\
\hline & ${ }^{4} \mathrm{D}_{31 / 2}^{\circ}$ & $\begin{array}{l}50210.80 \\
55706.57\end{array}$ & 490.72 & $\begin{array}{l}\text { 1. } 360 \\
\text { 1. } 413\end{array}$ & & ${ }^{2} I_{61 / 2}^{\circ}$ & 62980. 24 & & 1. 091 \\
\hline \multirow{4}{*}{$4 d^{4}\left(a^{3} \mathrm{P}\right) 5 p$} & $\left(y{ }^{4} \mathrm{D}_{01 / 2}^{\circ}\right.$ & 57319.55 & & 0. 200 & \multirow{3}{*}{$4 d^{4}\left(a^{3} \mathrm{~F}\right) 5 p$} & $\left(y^{4} \mathrm{~F}_{11 / 2}^{0}\right.$ & 63002.58 & \multirow{3}{*}{$\begin{array}{r}389.94 \\
-287.89 \\
678.47\end{array}$} & 0.899 \\
\hline & ${ }^{4} \mathrm{D}_{11 / 2}^{\circ}$ & 58140.75 & 821.20 & 1. 183 & & ${ }^{4} \mathrm{~F}_{21 / 2}^{\circ}$ & 63392.52 & & 1. 002 \\
\hline & ${ }^{4} \mathrm{D}_{21 / 2}^{\circ}$ & & & 1. 263 & & ${ }^{4} \mathrm{~F}_{31 / 2}^{\circ}$ & 63104. 63 & & 1. 060 \\
\hline & ${ }^{4} \mathrm{D}_{31 / 2}^{\circ}$ & 60702.16 & 135 & 1. 305 & & $\left({ }^{4} \mathrm{~F}_{41 / 2}^{\circ}\right.$ & 63783.10 & & \\
\hline \multirow{4}{*}{$4 d^{4}\left(a^{3} \mathrm{H}\right) 5 p$} & $\left(z^{4} \mathrm{H}_{31 / 2}^{\circ}\right.$ & 57892.06 & & 0. 710 & & $2_{31 / 2}^{\circ}$ & 63012. 24 & \multirow{4}{*}{$\begin{array}{r}-123.53 \\
35.81\end{array}$} & 1. 186 \\
\hline & ${ }^{4} \mathrm{H}_{41 / 2}^{\circ}$ & 58196. 68 & 004.02 & 0. 960 & & $\left(y^{4} \mathrm{G}_{21 / 2}\right.$ & 63041.47 & & 0.851 \\
\hline & ${ }^{4} \mathrm{H}_{51 / 2}^{\circ}$ & 58760.95 & 504.26 & 1. 110 & & ${ }^{4} \mathrm{G}_{31 / 2}^{\circ}$ & 62917. 94 & & 1. 004 \\
\hline & ${ }^{4} \mathrm{H}_{61 / 2}$ & 59491. 83 & 130.88 & 1. 206 & $p$ & ${ }^{4} \mathrm{G}_{41 / 2}$ & 62953.75 & & 1. 214 \\
\hline $4 d^{4}\left(a^{3} \mathrm{P}\right) 5 p$ & $z^{2} \mathbf{S}_{01 / 2}^{\circ}$ & 58527.00 & & 1. 651 & & ${ }^{4} \mathrm{G}_{51 / 2}$ & 63207.43 & & 1. 272 \\
\hline
\end{tabular}


Table 5. Odd terms of Mo II-Continued

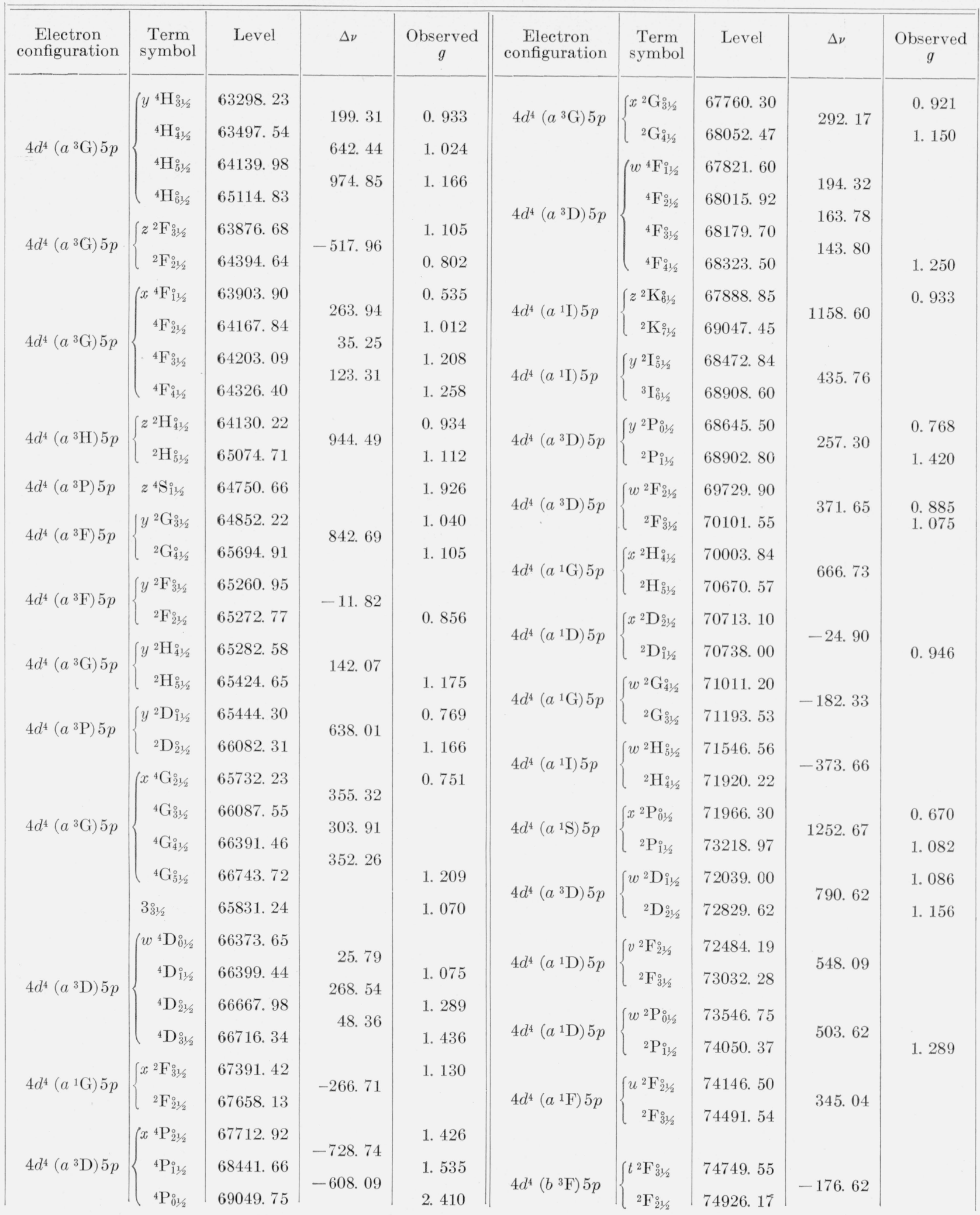


TABLE 5. Odd terms of Mo II-Continued

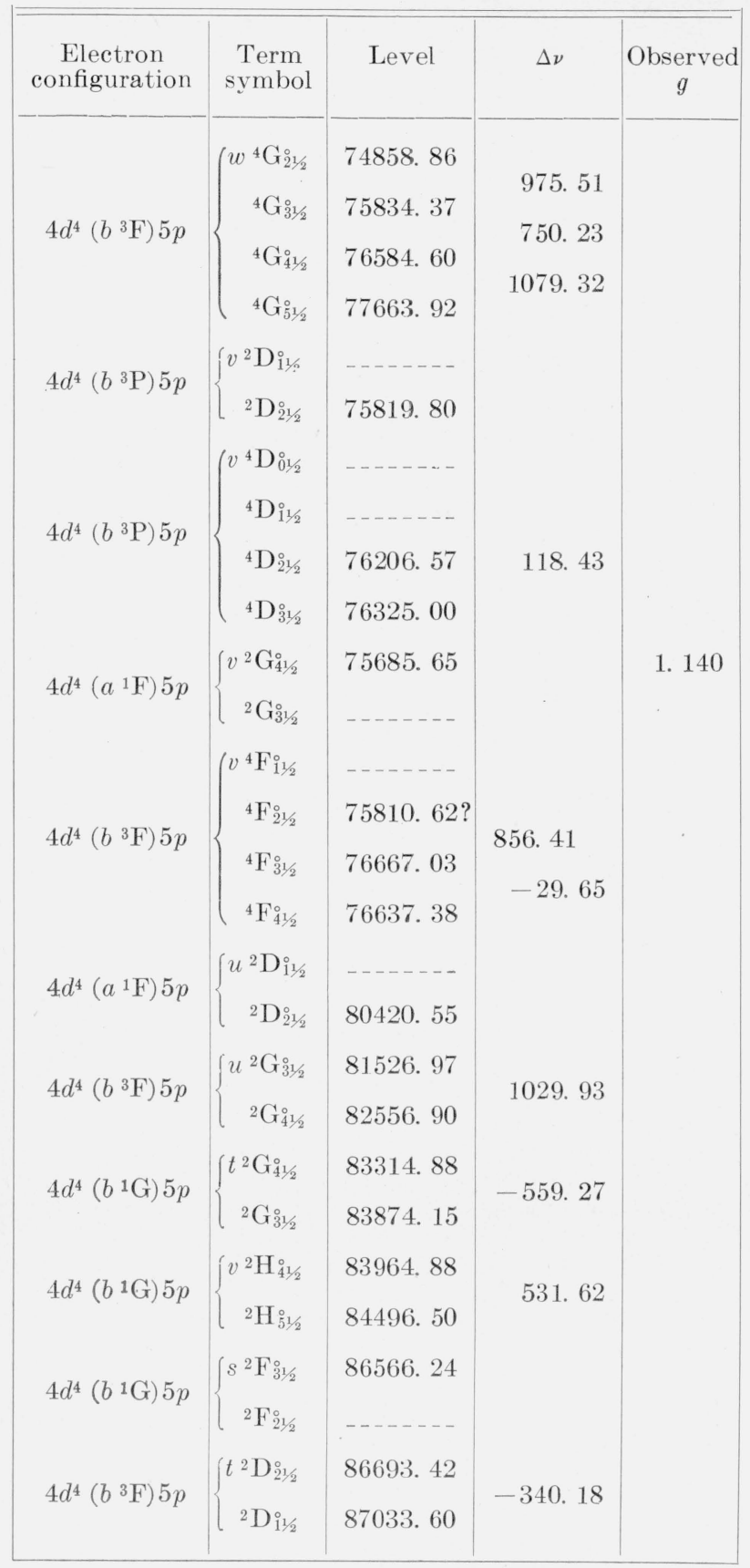

TABLE 6. Theoretical terms of Mo II

\begin{tabular}{|c|c|}
\hline $\begin{array}{c}\text { Electron } \\
\text { configuration } \\
1 s^{2} 2 s^{2} 2 p^{6} 3 s^{2} 3 p^{6} \\
3 d^{10} 4 s^{2} 4 p^{6}+\end{array}$ & Theoretical terms \\
\hline $4 d^{5}$ & 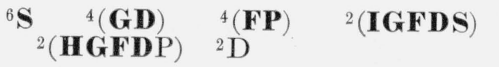 \\
\hline $4 d^{4} 5 s$ & 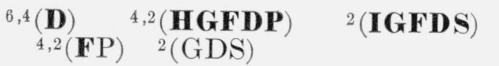 \\
\hline $4 d^{3} 5 s^{2}$ & ${ }^{4}(\mathrm{FP}) \quad{ }^{2}(\mathrm{HGFDP}) \quad{ }^{2} \mathrm{D}$ \\
\hline $4 d^{4} 5 p$ & 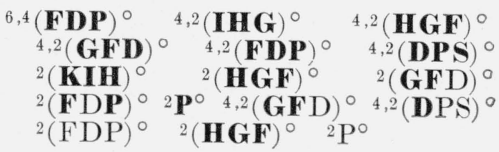 \\
\hline
\end{tabular}

Washington, September 30, 1957. 